\title{
A VARIATIONAL CLUSTER APPROXIMATION FOR THE HEISENBERG MODEL
}

\author{
Dissertation \\ zur Erlangung des mathematisch-naturwissenschaftlichen \\ Doktorgrades \\ "Doctor rerum naturalium" \\ der Georg-August-Universität Göttingen \\ im Promotionsprogramm ProPhys \\ der Georg-August University School of Science (GAUSS)
}

\author{
vorgelegt von \\ Stephan Filor \\ aus Bad Kreuznach
}

Göttingen, 2016 
- Prof. Dr. Stefan Kehrein, Institut für Theoretische Physik, Georg-August-Universität Göttingen

- PD Dr. Salvatore Manmana, Institut für Theoretische Physik, Georg-August-Universität Göttingen

MITGLIEDER DER PRÜFUNGSKOMMISSION:

- Referent:

Prof. Dr. Stefan Kehrein,

Institut für Theoretische Physik, Georg-August-Universität Göttingen

- Korreferent:

Prof. Dr. Andreas Honecker,

Laboratoire de Physique Théorique et Modélisation, Université de Cergy-Pontoise

- PD Dr. Salvatore Manmana,

Institut für Theoretische Physik, Georg-August-Universität Göttingen

- Prof. Dr. Reiner Kree, Institut für Theoretische Physik, Georg-August-Universität Göttingen

- Prof. Dr. Stefan Mathias

1. Physikalisches Institut, Georg-August-Universität Göttingen

- Prof. Dr. Michael Seibt

4. Physikalisches Institut, Georg-August-Universität Göttingen 
How come I end up where I started?

How come I end up where I went wrong?

Won't take my eyes off the ball again...

— Radiohead, 15 Step

To my family and friends,

for love, faith and soul...

To Thomas,

for opportunity, patience and support... 



\section{ABSTRACT}

In this thesis we present a novel variational cluster approximation for Heisenberg spin systems. It is based on the self-energy functional theory by Potthoff for fermionic and bosonic models with local interactions. To develop a similar method for spin systems, we derive a free energy functional which is the starting point of the approximation. Within this approximation, we find an analytical expression to evaluate the free energy by tiling the real system into a set of clusters.

We describe the technical details of the spin variational cluster approximation and the evaluation of the free energy. The method is tested for the antiferromagnetic spin- $1 / 2$ Heisenberg chain with first- and secondneighbour interactions. Thereby, we investigate the dependence of the approximation on cluster size and the choice of variational parameters. The opportunities and limitations as well as future applications of the method are thoroughly discussed. 



\section{PUBLICATIONS}

The following papers have been published in the context of this thesis:

- Stephan Filor and Thomas Pruschke:

"Variational cluster approximation to the thermodynamics of quantum spin systems."

In: New Journal of Physics, (2014), 16, 063059

- Stephan Filor and Thomas Pruschke:

"A self-energy functional approach for spin systems."

In: Journal of Physics: Conference Series, (2010), 200, 022007 



\section{CONTENTS}

1 INTRODUCTION 1

1.1 Electron Spin and Exchange Interaction . . . . . . . . 2

1.2 The Heisenberg Model . . . . . . . . . . . . . . . . . 4

1.3 Motivation of the Thesis . . . . . . . . . . . 6

1.4 Outline of the Thesis . . . . . . . . . . . . . . 10

2 DISCUSSION OF METHODS 11

2.1 Self-Energy Functional Theory . . . . . . . . . . . . 12

2.2 The Spin Diagram Technique . . . . . . . . . . . . 17

2.3 Operator Transformations . . . . . . . . . . . . . . . . 25

2.3.1 Fermionic Transformations . . . . . . . . . . 25

2.3.2 Bosonic Transformations . . . . . . . . . . . . . 28

2.4 The Resolvent SEFA . . . . . . . . . . . . . . . . . . . . . 31

3 THE SPIN VARIATIONAL CLUSTER APPROXIMATION 35

3.1 The Free Energy Functional . . . . . . . . . . . . 36

3.2 Luttinger-Ward functional for spin systems . . . . . . . . 41

3.3 The Spin VCA equations . . . . . . . . . . . . . . . . . . 45

3.4 Evaluation of the Spin VCA Equations . . . . . . . . . 48

3.5 Discussion of the SVCA Method . . . . . . . . . . 53

4 RESULTS OF THE SPIN VCA 57

4.1 Details on the Implementation of the SVCA . . . . . . . 58

4.2 Antiferromagnetic Spin Chain . . . . . . . . . . . . . . . . 63

4.3 Results for a Frustrated Model . . . . . . . . . . . . . . 68

5 CONCLUSION AND DISCUSSION 73

A SPIN-COHERENT STATES AND PATH INTEGRALS 79

A.1 Spin-Coherent States . . . . . . . . . . . . . . . . 79

A.2 The Spin Path Integral . . . . . . . . . . . . . 82

B SINGULAR MATRIX OF CORRELATION FUNCTIONS 87

B.1 The Singular Correlation Matrix . . . . . . . . . . . 87

B.2 Implications of the Singular Matrix . . . . . . . . . . 89

C THE INTERACTION MATRIX IN FOURIER REPRESENTATION 91

D SVCA EXTENSION TO VARIATIONAL MAGNETIC FIELDS 95

BIBLIOGRAPHY 99 



\section{INTRODUCTION}

The phenomenon of magnetism has been fascinating laymen and scholars alike for a long time. Materials where magnetic forces could be perceived surely were of the earliest interest [Mat06]. Such substances with a permanent or temporary field-induced magnetisation are often ferrorespectively ferrimagnets. Yet, there are other forms in which one can encounter the phenomenon - the classic ones being diamagnetism, paramagnetism and antiferromagnetism. In the latter one can indeed have magnetic ordering without a net macroscopic moment. Further and more exotic types could be added here [Hur82]. Real materials can actually show several of these phases, depending for example on temperature, pressure or applied fields.

The question is how one can explain or predict these abundant and fascinating magnetic properties by means of theoretical physics. The task is highly complicated and has been the study of many scientists. Magnetism in solids is a collective phenomenon, based on the interaction of electrons. Given the vast number of particles and mutual correlations in a macroscopic system, it is impossible to find an exactly solvable theory. Yet, a suitable approximation has to start with the ions and electrons that constitute a solid, their properties and interactions.

In this introduction we will first discuss the direct exchange of interacting electrons and then introduce the famous Heisenberg Hamiltonian which will be our model of choice. This is followed by a brief overview on how such a model can be treated, which finally leads to the motivation for this thesis. 


\subsection{ELECTRON SPIN AND EXCHANGE INTERACTION}

Each electron has an intrinsic angular momentum with a quantization $\hbar / 2$, its spin. ${ }^{1}$ This quantity is represented by the quantum mechanical operator $\overrightarrow{\mathbf{S}}=\left(\mathbf{S}^{x}, \mathbf{S}^{y}, \mathbf{S}^{z}\right)$. The eigenvalues of the $z$-component are $+1 / 2$ and $-1 / 2$. In a heuristic picture one can call the corresponding states $|\uparrow\rangle$ 'spin up' and $|\downarrow\rangle$ 'spin down'.

An electron acquires a magnetic moment due to its intrinsic angular momentum. So it couples to a magnetic field $\vec{h}$ which acts on the particle. The corresponding Hamiltonian is of the form $\vec{h} \cdot \vec{S}$ [Faz99]. This coupling has direct consequences on the energy levels of atoms. For example, Hydrogen consists of a single electron and proton. In the rest frame of the former the charged nucleus is moving. This gives rise to a magnetic field which couples to the spin of the electron. This so-called spin-orbit coupling leads to the fine structure of the energy levels of the hydrogen atoms. It is essentially based on the interaction of the electron spin with its orbital angular momentum.

In atoms with more than one electron the situation naturally becomes complex due to electron-electron interactions. It is very instructive to first consider the simple case of two electrons. ${ }^{2}$ If we neglect the environment, the Hamiltonian $\mathrm{H}$ can be assumed as consisting of the single particle terms $\mathbf{H}_{0}$ as well as the Coulomb interaction $\mathbf{H}_{\mathrm{C}}$. Suppose the electrons are in two orthogonal eigenstates $\psi_{\mathrm{a}}$ and $\psi_{\mathrm{b}}$ of $\mathbf{H}_{0}$ and only their spin can be varied. This means that one has four (anti-symmetrized) states, two with parallel - abbreviated $|\uparrow \uparrow\rangle$ and $|\downarrow \downarrow\rangle$ - and two with antiparallel spin - abbreviated $|\uparrow \downarrow\rangle$ and $|\downarrow \uparrow\rangle$. Of these only the parallel ones are eigenstates of the full Hamiltonian with its Coulomb interaction. On the other hand the elements of the transition $\left\langle\uparrow \downarrow\left|\mathbf{H}_{C}\right| \downarrow \uparrow\right\rangle$ and $\left\langle\downarrow \uparrow\left|\mathbf{H}_{C}\right| \uparrow \downarrow\right\rangle$ are finite. This essentially means that the Coulomb interaction mediates an exchange of the spins. One has to diagonalize the subspace of antiparallel spin to find the eigenstates of the full Hamiltonian. The new basis is determined by the total spin $S$ and its $\mathrm{z}$-component $\mathrm{m}$. Three states form a triplet with the same eigenenergy [Gri05]:

$$
|1,1\rangle=|\uparrow \uparrow\rangle, \quad|1,0\rangle=\frac{1}{\sqrt{2}}(|\uparrow \downarrow\rangle+|\downarrow \uparrow\rangle), \quad|1,-1\rangle=|\downarrow \downarrow\rangle,
$$

while the fourth is a singlet:

$$
|0,0\rangle=\frac{1}{\sqrt{2}}(|\uparrow \downarrow\rangle-|\downarrow \uparrow\rangle)
$$

In the case presented here of two electrons in orthogonal orbitals the energy of the triplet is lower than that of the singlet. This means that

1 For the remainder of this thesis we will set the reduced Planck constant $\hbar$ equal to one. The same holds for the Boltzmann constant $k_{B}$.

2 The two-electron problem is historically most interesting. Among others Heisenberg [Hei26] and Dirac [Dir26] gave important contributions. The discussion here follows [VV32] and [Faz99]. 
states with parallel spin and thereby ferromagnetism are favoured by the so-called direct exchange of the spins [Faz99]. This can be understood by taking the Pauli exclusion principle into account. It is forbidden for two fermions with equal quantum numbers to occupy the same position in real space. This leads to the exchange hole effect, which means that electrons with parallel spin tend to stay away from each other. Naturally this is not the case for anti-parallel configurations where the electrons differ in $\mathrm{m}$. Here, the particles can occupy the same position and so the mutual Coulomb repulsion is stronger which raises the energy level of the singlet state.

The Hamiltonian of the two-particle system can actually be expressed in terms of the electron spin operators [VV32]:

$$
\mathrm{H}=\text { constant }+2 \mathrm{~J} \overrightarrow{\boldsymbol{S}}_{\mathrm{a}} \cdot \overrightarrow{\boldsymbol{S}}_{\mathrm{b}},
$$

where the parameter $\mathrm{J}$ is the exchange integral which has already been mentioned as the transition element between the two anti-parallel states. It was first noted by Dirac that the Hamiltonian of two interacting electrons in orthogonal orbitals with variable spin can be written - bar a constant - in the form of coupled spin operators [VV32]. The expression (1.3) is called an exchange Hamiltonian.

It has to be emphasized that such a spin-spin-coupling is a direct consequence of the Coulomb interaction and the Pauli exclusion principle. It does not follow from the magnetic forces. Indeed, for the problem at hand - two electrons in an atom - they are small compared to the exchange integral and can be neglected [VV32].

The direct exchange process that we discussed is not the only one that leads to a Hamiltonian of the form (1.3). At least approximately it can be found in a large variety of situations. Such Hamiltonians generally can be written as:

$$
\mathbf{H}=\sum_{\mathrm{a}, \mathrm{b}} \mathrm{J}_{\mathrm{ab}} \overrightarrow{\mathbf{S}}_{\mathrm{a}} \cdot \overrightarrow{\mathbf{S}}_{\mathrm{b}}
$$

where the indices $a$ and $b$ refer intra-atomic orbitals. In this formulation states with parallel spin have lower energy if the exchange interaction $\mathrm{J}$ is negative. A Hamiltonian of that kind favours ferromagnetic behaviour. One such example is the direct exchange discussed above. If the J in (1.4) is positive, then anti-parallel spin states are favoured. Such antiferromagnetic interactions can be realized by a variety of processes. One example is the so-called kinetic exchange where the restriction of a single electron per orbital is lifted. Direct processes with non-orthogonal states also tend to be antiferromagnetic. Furthermore, there are indirect exchanges which involve a third orbital. These can exhibit interactions with a positive J [Faz99].

Naturally the situation becomes more complex if one investigates atoms with many electrons. The interplay of their spin and orbital angular momentum can lead to a net magnetic moment. Depending on the configuration of the electrons the spin attributed to the atom can be 
bigger than 1/2 [Faz99]. We will not discuss this in detail here. Instead, we will now turn to a lattice system and discuss interacting electrons in a solid.

\subsection{THE HEISENBERG MODEL}

To describe an extended respectively macroscopic system one needs a proper model that captures the relevant features. According to the Bohr - van Leeuwen theorem, magnetism in solids is a quantum mechanical effect [VV32]. As was already stated, it is essentially a collective phenomenon of correlated electrons. The simple direct exchange discussed in the previous section showed that spin-spin couplings arise from interacting particles.

It is often a good approximation to assume that the ions in a solid are fixed at certain positions, which constitute a background for itinerant electrons in conduction bands. In such a lattice representation the fermions can 'hop' from one site to another. The simplest and yet very successful many-body Hamiltonian to describe interacting spinful fermions is the Hubbard model [Hub65; Faz99]. Here, the kinetic energy appears in the form of a hopping between the sites while the repulsive interaction term favours localization of the particles. If the system has one narrow conduction band the Hamiltonian can be written as:

$$
\mathbf{H}=-\mathrm{t} \sum_{i, j} \sum_{\sigma}\left(\mathbf{c}_{i \sigma}^{\dagger} \mathbf{c}_{j \sigma}+\mathbf{c}_{j \sigma}^{\dagger} \boldsymbol{c}_{i \sigma}\right)+\mathrm{U} \sum_{i} \mathbf{n}_{i \uparrow} \mathfrak{n}_{i \downarrow},
$$

where the $\boldsymbol{c}_{i \sigma}^{\dagger}$ and $\boldsymbol{c}_{i \sigma}$ are fermionic creation and annihilation operators, while the $\boldsymbol{n}_{i \sigma}$ measure the particle occupation for a given spin quantum number $\sigma=\uparrow, \downarrow$ at a specific site $i$. In addition to the lattice geometry the model is determined by the hopping parameter $t$ and the interaction $\mathrm{U}$ as well as the amount of electrons in the system. This quantity per site is called filling $n$ and can vary between 0 and 2 in the one-band Hubbard model. Fractional $n$ can arise by a so-called doping of the lattice with different types of atoms. This Hamiltonian has been studied extensively [Bae ${ }^{+}$95; Tas98]. The model can solved exactly in 1D [Ess $\left.{ }^{+} 05\right]$, yet it is an ongoing focus of research for $\mathrm{D}>1$.

The half-filled $n=1$ case is especially interesting for magnetism in the limit of large interaction $\mathrm{U}$. Here, a transition to the insulating Mott phase occurs where the electrons become localized at the sites respectively atoms. Yet, exchange processes are mediated by the U-coupling which can be approximately described by the so-called Heisenberg Hamiltonian [Faz99; Mat06]:

$$
\mathrm{H}=\sum_{i, j} J_{i j} \overrightarrow{\mathbf{S}}_{i} \cdot \overrightarrow{\mathrm{S}}_{j},
$$

with interactions $J$ between neighbouring sites $i$ and $j$. This is the lowenergy effective Hamiltonian of the half-filled Hubbard model (1.5) in 
the large-U limit. More precisely this is only the leading term of the approximation, higher orders include quadruple spin couplings or secondneighbour interactions [Faz99].

The Hamiltonian (1.6) is similar to (1.4), yet one has to note that the direct exchange there is based on correlations within an atom, while the processes described by the Heisenberg model are inter-atomic. The coupling $\mathrm{J}$ is given by $4 \mathrm{t}^{2} / \mathrm{U}$ where $\mathrm{t}$ is the kinetic energy parameter from (1.5). The interaction is antiferromagnetic because the exchange arises from virtual hopping processes between neighbouring antiparallel spins which lead to an energy gain. Thereby intermediate states are formed where both electrons happen to be on a single site. Due to the Pauli principle this is forbidden for electrons with parallel spin. This effectively lowers the energy of states with antiparallel spins [SS06].

If the filling $n$ of the Hubbard model (1.5) is less than 1 the system can be described by the effective $t-J$-model for itinerant electrons [Faz99]. As for $n=1$, spin-spin exchange terms enter the Hamiltonian, but the particles are additionally allowed to hop between sites, whereas doubleoccupation is excluded. Although models like these which incorporate itinerant electrons are of importance in understanding magnetic properties and superconductivity for some metals, we restrict ourselves in this work to insulating phases and effective Heisenberg Hamiltonians.

The one-band Hubbard model is not the only many-body system that can be approximated via spin-spin coupling terms [SS06; Faz99]. For example, Heisenberg Hamiltonians with $S=1 / 2$ are found also for some systems with more than one orbital. These may also lead to ferromagnetic coupling due to higher order exchanges. Effective models with larger spins are possible depending on filling and the electron configuration of the lattice atoms.

The Hamiltonian (1.6) is isotropic. When spin-orbit coupling or crystalfield splitting in the atoms is taken into account for the electron model, one may end up with an exchange anisotropy [Faz99]. Essentially, a large variety of materials can be modelled by effective Hamiltonians of the form (1.6) [Mat06].

We conclude this section with a short discussion of mathematical properties of the spin operators and the Heisenberg model. ${ }^{3}$

An operator $\overrightarrow{\mathbf{S}}$ can have integer or half-integer total spin $\mathrm{S}$. The corresponding Hilbert space is $(2 \mathrm{~S}+1)$-dimensional. The eigenstates of $\overrightarrow{\boldsymbol{S}}^{2}$ and $S^{z}$ are determined by the quantum numbers $S$ and $m=-S, \ldots, S$. The latter is the eigenvalue of the $z$-component, so:

$$
\mathrm{S}^{z}|\mathrm{~S}, \mathrm{~m}\rangle=\mathrm{m}|\mathrm{S}, \mathrm{m}\rangle,
$$

holds. One can also define the ladder operators:

$$
\mathbf{S}_{ \pm}=\mathbf{S}_{\mathrm{x}} \pm \mathrm{i} \mathbf{S}_{\mathrm{y}}
$$

3 A thorough treatment of spin and the mathematical aspects of the Heisenberg Hamiltonian can be found in [CTDL91] and [NR09]. 
which raise or lower $m$ by one and act on the spin states in the following way:

$$
S_{ \pm}|S, m\rangle=\sqrt{S(S+1)-m(m \pm 1)}|S, m \pm 1\rangle .
$$

The components of a spin operator $\overrightarrow{\mathbf{S}}$ form a Lie algebra with the following commutation relations:

$$
\left[\boldsymbol{S}_{\mathrm{k}}, \boldsymbol{S}_{\mathrm{l}}\right]=\mathrm{i} \sum_{\mathrm{m}} \epsilon_{\mathrm{klm}} \boldsymbol{S}_{\mathrm{m}}
$$

where the Levi-Civita tensor $\epsilon$ is used and the indices denote elements of the ordered triple $(x, y, z)$. In a lattice system operators acting on different sites commute. So one can use products of single site states $|S, m\rangle$ as a basis for many-spin Hamiltonians. Since they act in this Hilbert space via the relations (1.9), it is convenient to express the Heisenberg model (1.6) in terms of the ladder operators (1.8):

$$
\mathbf{H}=\sum_{i j}\left[J_{i j}^{z z} \boldsymbol{S}_{i}^{z} \mathbf{S}_{j}^{z}+\frac{1}{2} J_{i j}^{-+}\left(\boldsymbol{S}_{i}^{+} \boldsymbol{S}_{j}^{-}+\mathbf{S}_{i}^{-} \boldsymbol{S}_{j}^{+}\right)\right] .
$$

If the Hamiltonian is isotropic, $\mathrm{J}_{\mathrm{ij}}^{z z}$ and $\mathrm{J}_{\mathrm{ij}}^{-+}$are equal. This changes in the case of anisotropic exchange. We will in this thesis frequently refer to the $(z z)$-term of (1.11) as longitudinal interaction while the (+-)-terms are designated transversal. The Heisenberg Hamiltonian (1.11) can also be varied by adding certain terms. For example, a magnetic field applied in the $z$-direction comes in the form of a Zeeman term $\sum_{i} h S_{i}^{z}$. If the system exhibits single-ion anisotropy one can add a term like $\sum_{i}\left(\mathbf{S}_{i}^{z}\right)^{2}$ [Faz99]. Other possible corrections like higher order ring exchanges or the Dzyaloshinskii-Moriya interaction will not be considered in this thesis [LMM11].

\subsection{MOTIVATiOn OF THE THESis}

As was discussed in the previous section, magnetic materials can in many cases be represented well by models of localized spins interacting via exchange couplings. A simple one is the Heisenberg Hamiltonian (1.11). The physics is well-known for the one-dimensional case [Klu93; EAT94; Klu98; Joh ${ }^{+} 00 ;$ Tak09], and for dimensions D $\geqslant 4$, where a Weiss mean-field theory is applicable [ID89]. Moreover, for first-neighbour exchange and simple lattices one can use highly efficient Monte-Carlo simulations to investigate the static and dynamic properties of the Heisenberg model [San10].

In general ferromagnetic Hamiltonians are easier to treat, especially in the low-temperature regime. The ground-state of a lattice that favours parallel spin is exactly known - the energy is minimized if all spins are 'pointing' in a specific direction. In an isotropic Hamiltonian symmetry 
breaking has to occur since it is rotationally invariant. Here, the groundstate is degenerate regarding spatial directions, yet only one alternative is realized. Starting with one such solution the low-lying excitations of the ferromagnetic Heisenberg model can in principle be successfully treated by using spin wave theories [Faz99].

For antiferromagnetic exchange the situation is different. Here, the ground-state is not given by strictly anti-parallel spins, which would be the correspondence to the ferromagnetic case. Such a classical Néel state on a bipartite lattice can be described by two sub-lattices with all spin up respectively all down configuration. The magnetization on each of these is finite yet the total sum adds up to zero.

The energy for a Néel state would be minimized for the $\boldsymbol{S}_{i}^{z} \mathbf{S}_{j}^{z}$-part of (1.11), which corresponds to the Ising model. Taking the transversal spin-flip interactions into account, the ground-state is subject to quantum fluctuations [Faz99]. Yet, there exists Néel like order in some models. An example would be the $S=1 / 2$ chain with nearest-neighbour interaction. Here, one finds algebraic long-range order in the groundstate. The system is gapless and the elementary excitations are spinons [FT81; MK04]. The $S=1$ spin chain with antiferromagnetic interaction shows a very different behaviour. The ground state is a degenerate singlet and the magnetization on each site is zero. Short-range spin correlations dominate the model. Additionally, a gap is found between the ground-state and the first excitations, which cannot be described by spin wave theory in a satisfactory way [Mat06; MK04].

So, these two spin chain models differ profoundly: ${ }^{4}$ The $S=1 / 2$ case has a ground-state phase which one would classically call 'antiferromagnetic', while for $S=1$ the system is treated more successful using bonds between the spins rather than spins itself [Mat06]. Here, the groundstate is given by an AKLT valence bond state [Aff ${ }^{+}$87; $\mathrm{Aff}^{+}$88].

For two-dimensional antiferromagnetic Heisenberg models the situation is even more diverse. A variety of phases can evolve depending on lattice structure and exchange parameters - including Néel like order, paramagnetic ground-states or spin liquids [RSH04; LMM11].

The most intriguing problems are the so-called frustrated systems. Frustration is given if two or more interactions compete, each of them trying to enforce a different order, which can lead to novel ground-states and phases. Such systems are an active field of research and pose challenges for theoretical description [LMM11]. Frustration can be realized in various ways, for example in the presence of longer-ranged antiferromagnetic exchange. Fig. 1.1 shows the one-dimensional $J_{1}-J_{2}$-chain and a square lattice, both featuring second-nearest neighbour interactions. Here, the case $J_{1}=2 J_{2}$ is the most interesting since frustration is likely to be largest in he model [MG69; LMM11].

For the chain this point is called Majumdar-Ghosh model. Here, the

4 Haldane's famous conjecture states that one-dimensional antiferromagnets with arbitrary integer spin have a degenerate ground state and no long-range order. They also exhibit a gapped excitation spectrum. More on this can be found in [Hal83] and [AL86]. 


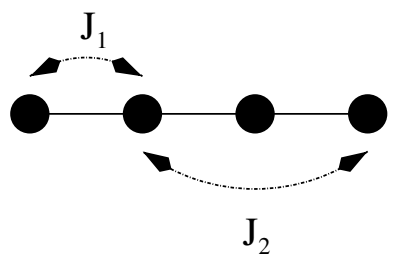

(a)

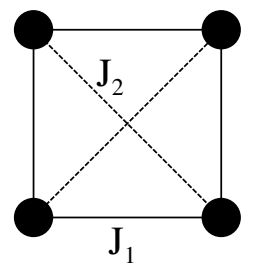

(b)

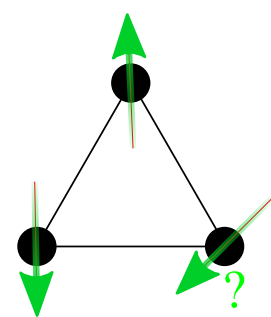

(c)

Figure 1.1: The graphic depicts segments of three frustrated lattices. (a) and (b) have two competing antiferromagnetic interactions. The spin chain (a) is the $\mathrm{J}_{1}-\mathrm{J}_{2}$ model. Both (a) and the square lattice (b) exhibit second-neighbour exchange. The example (c) is a triangle with antiferromagnetic interactions between the spins. With these one can build frustrated systems like the Kagome lattice. They are geometrically frustrated since there is no possibility to align all spins antiparallel. This is highlighted by the green arrows.

degenerate ground-state is exactly given by the product of quantum dimer states. These are singlet configurations between two neighbouring spins [MG69]. Like in many frustrated systems, the order is rather short-ranged even for low temperatures.

Models with competing first- and second-neighbour interactions are an interesting starting point for theoretical descriptions. Yet, more common in real systems is geometrical frustration, which is a consequence of the lattice structure. Here, a possible Néel order is already destabilized for first-neighbour interactions only. An example would be a triangular lattice, pictured in fig. 1.1. A general antiparallel alignment of neighbouring spins is not possible in this case due to the arrangement of the sites and the associated exchange interactions. Other examples for geometrically frustrated systems are the two-dimensional Kagome and the threedimensional pyrochlore lattice [LMM11].

For models with frustration the Monte-Carlo method is often plagued by a so-called sign problem and reliable results for low-temperature properties cannot be obtained [TW05]. There are exceptions like spin chains or the fully frustrated spin ladder [Nak98; ADP15; $\left.\mathrm{Hon}^{+} 16\right]$.

Though, in the presence of frustration the sign problem is typically severe, especially in higher-dimensional lattices. Other prominent examples of numerical methods that have been applied to frustrated spin models are the density-matrix renormalization group, exact diagonalization and high-temperature series expansions [LMM11]. Yet, these fail to capture the low-temperature behaviour in an appropriate way.

A further possible approach to a theoretical treatment of frustrated spin systems are cluster based approximations. Here, one uses certain spatial sub-systems of the original lattice which are mathematically easier accessible. Heuristically speaking, to work on the basis of clusters might be especially useful for frustrated systems, where often short- 
ranged correlations dominate the low-temperature behaviour. Yet, to simulate the thermodynamical limit the sub-systems have to be embedded into an effective dynamical environment.

For fermionic systems such cluster techniques are well established $\left[\mathrm{Mai}^{+} 05\right]$. In the case of spin Hamiltonians, the simplest example would be the Weiss mean-field theory with single sites in a static effective magnetic field [ID89]. A cluster perturbation theory has also been proposed for the Heisenberg model [OV04]. Embedded cluster approaches for quantum spin models in general are still in the early stages of development. Such approximations might have the potential to be suitable for frustrated systems, but much work is needed to see if they can live up to it.

A starting point of cluster theories for itinerant fermions is the selfenergy functional approach (SEFA) proposed by Potthoff [Pot03b]. It was originally developed for spinless fermions in a Hubbard model of type (1.5). The approach is based on the observation going back to Luttinger, Ward, Baym and Kadanoff that the free energy can be formally represented as a functional of the fermionic Green function respectively self-energy. It includes a non-trivial part called Luttinger-Ward functional [BK61], which only depends on the structure of the interaction but not on the kinetic energy of the fermions. This feature allows to create well-defined approximations for models with strictly local interactions by replacing the lattice with a collection of clusters, which can then be treated exactly. These so-called variational cluster approaches (VCA) have been used to study various models for interacting fermion systems [Sen08] as well as the Bose-Hubbard-model [KD06].

The method introduced by Potthoff strongly relies on the possibility to represent the free energy as a functional of the self-energy, with the interaction strictly separated from the non-interacting part. This property is in turn based on a linked-cluster expansion for the free energy involving Wick's theorem, which relies on the standard Bose or Fermi commutation relations among the field operators constituting the noninteracting system [FW71]. However, spin operators form another algebra, given in (1.10), and so the standard formalism does not work. Nevertheless, due to the reasons mentioned above it would be interesting to have an analogous method for Heisenberg Hamiltonians. It would open a new way to tackle the problem of frustrated spin systems. 


\subsection{OUTLINE OF THE THESIS}

The goal of this thesis is to devise an approach to Heisenberg models akin to Potthoff's SEFA and to subsequently find means of establishing a variational cluster approximation. Indeed, we will propose such a formulation in this work and test it on simple spin models.

The next chapter 2 collects several methods and approaches to the problem. It starts with an introduction of Potthoff's SEFA and the VCA for fermionic systems. The following sections feature discussions of several ideas how an analogous formulation for spin Hamiltonians could be established. However, all of these approaches face certain problems. Parts of section 2.4 have been previously published in [FP10].

In chapter 3 a coherent-state representation will be introduced for spin operators which leads to a functional derivation of the correlators. Within this formulation one can derive an expression which has the structure of a Luttinger-Ward functional. This will serve as the starting point for an approximation which is based on a separation of the full system into clusters - the spin VCA. The derivation in this chapter is a revised and expanded version of a scheme presented in [FP14].

The approximation is tested for the antiferromagnetic $S=1 / 2$ Heisenberg chain in chapter 4 . These results were published in [FP14]. This chapter additionally features a section where the utilization of the method for the frustrated $\mathrm{J}_{1}-\mathrm{J}_{2}$ model is discussed.

The thesis concludes with chapter 5. It is comprised of a discussion of the features and deficiencies of the spin VCA as well an overview over the possibilities of further development.

Finally, certain additional topics are treated in the appendices A-C. They are important for specific mathematical aspects of the thesis, but not general enough to appear in the main text. Additionally, appendix $\mathrm{D}$ introduces a possible yet preliminary solution to the problem of using local fields within the spin VCA. 


\section{DISCUSSION OF METHODS}

This chapter is dedicated to some methods and approaches which are important in the context of developing a variational cluster approximation for spin systems. As was already discussed in chapter 1 , the idea for this endeavour is based on the self-energy functional approach (SEFA). Since several of its concepts appear in later chapters, we will give a short introduction to the SEFA in section 2.1.

Prior to the approach for a spin VCA which is laid out in detail in this thesis, there have been three other ideas which we examined and tested. The first and somehow natural strategy would be to find an analogue to the functional of Baym and Kadanoff for fermions in a perturbative way. Section 2.2 gives a short overview to a spin diagram technique which originally seemed a good candidate to deliver such a relation. It proved not to be successful, but nevertheless a spin self-energy can be defined which becomes important for the spin variational cluster approximation in chapter 3.

Potthoff's SEFA was developed for fermionic and also bosonic operators. A straightforward idea for Heisenberg systems would be to directly make use of the established formalism by expressing the spins as such degrees of freedom. Thus, the third section discusses possible operator transformations.

A third approach is based on the resolvent of a given Hamiltonian. This quantity is easily defined for spin systems and one can even find a suitable functional for the free energy. A formal variational principle in the spirit of the SEFA is possible, yet it is not practicable enough for reasonable computations. Parts of the corresponding discussion in section 2.4 have been published previously in [FP10]. 


\subsection{SELF-ENERGY FUNCTIONAL THEORY}

In this section we will give an introduction to the self-energy functional approach (SEFA) which was proposed by Potthoff [Pot03b; Pot03a] and the subsequent variational cluster approximation (VCA). They were originally derived for fermionic systems, in particular the Hubbard model with on-site interactions. As was already stated in chapter 1 this method allows to embed cluster systems into a dynamical environment. A detailed review of the SEFA can be found in [Pot12a].

The method yields a dynamical variational principle for a thermodynamical potential $\Omega$ of a fermionic or bosonic system, namely $\delta \Omega[\boldsymbol{\Sigma}]=0$ [Pot03b; Pot12b]. Here, $\Omega$ is a functional of the self-energy $\Sigma$. This dynamical quantity as well as the potential itself is formally given by the variational principle. Naturally, for any practicable purposes approximations have to be made. The special property of the SEFA is that it restricts the domain of the self-energy and leaves the functional form $\Omega$ intact. This allows to make systematic approximations which guaranties thermodynamical consistency [Pot12a].

A lattice system of interacting electrons can be described by the Hubbard model which was already introduced in chapter 1.2:

$$
\begin{aligned}
\mathbf{H} & =\mathbf{H}_{0}(\mathbf{t})+\mathbf{H}_{\text {int }}(\mathbf{u}) \\
& =\sum_{\alpha \beta} t_{\alpha \beta} \mathbf{c}_{\alpha}^{\dagger} \mathbf{c}_{\beta}+\sum_{\alpha \beta \gamma \delta} u_{\alpha \beta \gamma \delta} \mathbf{c}_{\alpha}^{\dagger} \mathbf{c}_{\beta}^{\dagger} \mathbf{c}_{\gamma} \mathbf{c}_{\delta} .
\end{aligned}
$$

The Hamiltonian is parametrized by the hopping $t$ and the interaction $\mathrm{U}$. Note that this formulation of the Hubbard model is more general than (1.5). The Greek index denotes an arbitrary set of quantum numbers. For the SEFA it is yet important that the interaction $\mathbf{U}$ is spatially local.

The single-particle Green function of the system $G_{\alpha \beta}(\omega)$ is a frequency dependent dynamical quantity which provides the spectrum of one- particle excitations. Since it is dependent on the parameters of the model it can be denoted in compact matrix form as $\mathbf{G}_{\mathbf{t}, \mathbf{u}}$ and the free Green function with $\mathbf{U}=0$ subsequently is written as $\mathbf{G}_{\mathbf{t}, 0}$. With this one can define the self-energy $\boldsymbol{\Sigma}_{\mathrm{t}, \mathrm{u}}$ using Dyson's equation [AGD63]:

$$
\mathbf{G}_{\mathbf{t}, \mathbf{U}}=\left(\mathbf{G}_{\mathbf{t}, 0}^{-1}-\mathbf{\Sigma}_{\mathbf{t}, \mathbf{U}}\right)^{-1}
$$

The self-energy can be derived diagrammatically via perturbation theory. It vanishes if the interaction $\mathrm{U}$ is zero [AGD63]. One has to note the important aspect that the self-energy can generally be assumed to be more local than the Green function [Pot12a].

The grand potential $\Omega_{\mathfrak{t}, \mathrm{u}}$ is a central static quantity of a system described by a Hamiltonian (2.1) with conserved total particle number. Many aspects of its thermodynamics are determined by $\Omega_{t}, \mathbf{u}$ and its derivatives, for example with respect to the temperature $T$ or the chemical potential $\mu$ [Kar07]. 
The grand potential can be written as a functional of the Green function. This connection between static and dynamical quantities of a system is known as the Baym-Kadanoff functional [LW60; BK61; Bay62]:

$$
\tilde{\Omega}_{\mathbf{t}, \mathbf{u}}[\tilde{\mathbf{G}}]=\tilde{\Phi}_{\mathbf{u}}[\tilde{\mathbf{G}}]+\operatorname{Tr} \ln \tilde{\mathbf{G}}-\operatorname{Tr}\left(\tilde{\Sigma}_{\mathbf{u}}[\tilde{\mathbf{G}}] \tilde{\mathbf{G}}\right),
$$

where the trace is given by $\operatorname{Tr}(\ldots)=T \sum_{n} \exp \left(i \omega_{n} 0^{+}\right) \operatorname{tr}(\ldots)$ and goes over Matsubara frequencies as well as the indices introduced above. The so-called conserving approximations are based on the Baym-Kadanoff functional [BK61].

In (2.3), $\tilde{\Phi}_{\mathbf{u}}[\tilde{\mathbf{G}}]$ is the Luttinger-Ward functional [LW60]. It was originally derived perturbatively as the infinite series of so-called 'skeleton' diagrams, namely diagrams with no self-energy insertions where all free Green functions are replaced by interacting ones. It was later shown that this Luttinger-Ward functional can also be formally constructed using several important properties it exhibits [Pot06a].

Notably, when evaluated at the exact Green function one finds the quantity $\tilde{\Phi}_{\mathbf{u}}\left[\mathbf{G}_{\mathbf{t}, \mathbf{U}}\right]=\Phi_{\mathbf{t}, \mathbf{u}}$ and (2.3) yields the grand potential:

$$
\tilde{\Omega}_{\mathbf{t}, \mathbf{u}}\left[\mathbf{G}_{\mathbf{t}, \mathbf{u}}\right]=\Omega_{\mathbf{t}, \mathbf{u}}=\Phi_{\mathbf{t}, \mathbf{u}}+\operatorname{Tr} \ln \mathbf{G}_{\mathbf{t}, \mathbf{u}}-\operatorname{Tr}\left(\boldsymbol{\Sigma}_{\mathbf{t}, \mathbf{u}} \mathbf{G}_{\mathbf{t}, \mathbf{u}}\right) .
$$

The functional derivative of the Luttinger-Ward functional with respect to the Green function is given by:

$$
\frac{\delta \tilde{\Phi}_{\mathbf{U}}[\tilde{\mathbf{G}}]}{\delta \tilde{\mathbf{G}}}=\mathrm{T} \cdot \tilde{\Sigma}_{\mathbf{u}}[\tilde{\mathbf{G}}]
$$

If this functional is evaluated at the physical Green function one finds the self-energy of the system via $\tilde{\Sigma}_{\mathbf{u}}\left[\mathbf{G}_{\mathbf{t}, \mathbf{u}}\right]=\boldsymbol{\Sigma}_{\mathbf{t}, \mathrm{u}}$.

The Luttinger-Ward functional is universal in the sense that the functional $\tilde{\Phi}_{\mathbf{u}}[\ldots]$ is only dependent on the interaction $\mathbf{U}$ and not on the one-particle parameters $\mathbf{t}$ [Pot12a]. This property is inherited by the selfenergy functional $\tilde{\Sigma}_{\mathbf{u}}[\tilde{\mathbf{G}}]$ through (2.5). Moreover, $\tilde{\Phi}_{\mathbf{U}}[\tilde{\mathbf{G}}]$ itself vanishes for $\mathbf{U}=0$, the non-interacting case.

Using the variational principle $\delta \tilde{\Omega}_{\mathbf{t}, \mathbf{u}}[\tilde{\mathbf{G}}] / \delta \tilde{\mathbf{G}}=0$ for the Baym-Kadanoff functional (2.3) and taking (2.2) and (2.5) into account one finds $\tilde{\mathbf{G}}^{-1}-\mathbf{G}_{\mathbf{t}, 0}^{-1}+\tilde{\mathbf{\Sigma}}_{\mathbf{u}}[\tilde{\mathbf{G}}]=0$. This relation is true if Dyson's equation holds. Thus, the grand potential functional (2.3) is stationary at the physical value of the systems' Green function.

For developing the SEFA one now has to set up such a variational principle with regards to the self-energy. First, a functional $\tilde{\mathbf{G}}_{\mathbf{U}}[\tilde{\boldsymbol{\Sigma}}]$ is introduced where one assumes that $\tilde{\Sigma}_{\mathbf{u}}[\tilde{\mathbf{G}}]$ is locally invertible [Pot03b]. ${ }^{1}$ With this a Legendre transform of the Luttinger-Ward functional can be constructed:

$$
\tilde{\mathrm{F}}_{\mathbf{U}}[\tilde{\boldsymbol{\Sigma}}]=\tilde{\Phi}_{\mathbf{U}}\left[\tilde{\mathbf{G}}_{\mathbf{U}}[\tilde{\Sigma}]\right]-\operatorname{Tr}\left(\tilde{\Sigma} \tilde{\mathbf{G}}_{\mathbf{U}}[\tilde{\Sigma}]\right)
$$

1 In a recent publication [KFG15], the authors show that $\tilde{\Sigma}_{\mathbf{U}}[\tilde{\mathbf{G}}]$ is not always singlevalued, but in some cases has an additional unphysical branch. If this has consequences for the inversion $\tilde{\mathbf{G}}_{\mathbf{U}}[\tilde{\boldsymbol{\Sigma}}]$ and the SEFA is still an ongoing discussion. 
The functional derivative is directly given using (2.5) as:

$$
\frac{\delta \tilde{\mathrm{F}}_{\mathbf{U}}[\tilde{\boldsymbol{\Sigma}}]}{\delta \tilde{\boldsymbol{\Sigma}}}=-\mathrm{T} \cdot \tilde{\mathbf{G}}_{\mathbf{U}}[\tilde{\boldsymbol{\Sigma}}] .
$$

Finally one can define (2.3) as a functional of the self-energy:

$$
\tilde{\Omega}_{\mathbf{t}, \mathbf{U}}[\tilde{\boldsymbol{\Sigma}}]=\tilde{\mathrm{F}}_{\mathbf{U}}[\tilde{\boldsymbol{\Sigma}}]+\operatorname{Tr} \ln \left(\mathbf{G}_{\mathbf{t}, 0}^{-1}+\tilde{\mathbf{\Sigma}}\right)^{-1}
$$

where the Dyson equation (2.2) and the Legendre transform (2.6) was used. This new functional is stationary for the physical self-energy of the system, as can be easily seen when performing the functional derivative and taking (2.7) into account. The variational principle $\delta \tilde{\Omega}_{\mathbf{t}, \mathbf{u}}[\tilde{\boldsymbol{\Sigma}}] / \delta \tilde{\boldsymbol{\Sigma}}=0$ is the starting point for the SEFA.

Naturally, approximations have to be made since the functional $\tilde{F}_{\mathbf{U}}[\tilde{\boldsymbol{\Sigma}}]$ in (2.8) can not be computed exactly for any reasonable system. Yet, as the Luttinger-Ward functional it is universal and only depends on the interaction parameters $\mathbf{U}$. The central idea of the SEFA is to introduce a reference system $\mathbf{H}_{0}\left(\mathbf{t}^{\prime}\right)+\mathbf{H}_{\text {int }}(\mathbf{U})$ which has the same interaction as the original model but a different non-interacting part with one-particle parameters $t^{\prime}$. The self-energy functional for this system would be:

$$
\tilde{\Omega}_{\mathbf{t}^{\prime}, \mathbf{U}}[\tilde{\mathbf{\Sigma}}]=\tilde{\mathrm{F}}_{\mathbf{U}}[\tilde{\boldsymbol{\Sigma}}]+\operatorname{Tr} \ln \left(\mathbf{G}_{\mathbf{t}^{\prime}, 0}^{-1}+\tilde{\mathbf{\Sigma}}\right)^{-1} .
$$

As the Legendre transformed Luttinger-Ward functional $\tilde{F}_{\mathbf{U}}[\tilde{\Sigma}]$ is the same for both systems, it can be eliminated by combining (2.8) and (2.9):

$$
\begin{aligned}
\tilde{\Omega}_{\mathbf{t}, \mathbf{u}}[\tilde{\boldsymbol{\Sigma}}]= & \tilde{\Omega}_{\mathbf{t}^{\prime}, \mathbf{U}}[\tilde{\boldsymbol{\Sigma}}]+\operatorname{Tr} \ln \left(\mathbf{G}_{\mathbf{t}, 0}^{-1}+\tilde{\mathbf{\Sigma}}\right)^{-1} \\
& -\operatorname{Tr} \ln \left(\mathbf{G}_{\mathbf{t}^{\prime}, 0}^{-1}+\tilde{\Sigma}\right)^{-1} .
\end{aligned}
$$

This functional formulation is exact and, thus, still not solvable exactly. The problem basically has been shifted to $\tilde{\Omega}_{\mathbf{t}^{\prime}, \mathbf{U}}[\tilde{\Sigma}]$, which contains the full complexity of the problem. However, to the functional (2.10) the main approximation of the SEFA can be applied. Suppose the reference system is much simpler than the original one and its self-energy $\Sigma_{t, U}$ can be computed exactly. Now, this quantity can be inserted in (2.10) and using $\tilde{\Omega}_{\mathbf{t}^{\prime}, \mathbf{U}}\left[\tilde{\Sigma}_{\mathbf{t}^{\prime}, \mathbf{U}}\right]=\Omega_{\mathbf{t}^{\prime}, \mathbf{U}}$ as well as Dyson's equation one ends up with [Pot12a]:

$$
\tilde{\Omega}_{\mathbf{t}, \mathbf{u}}\left[\boldsymbol{\Sigma}_{\mathbf{t}^{\prime}, \mathbf{U}}\right]=\Omega_{\mathbf{t}^{\prime}, \mathbf{U}}+\operatorname{Tr} \ln \left(\mathbf{G}_{\mathbf{t}, 0}^{-1}+\boldsymbol{\Sigma}_{\mathbf{t}^{\prime}, \mathbf{U}}\right)^{-1}-\operatorname{Tr} \ln \mathbf{G}_{\mathbf{t}^{\prime}, \mathbf{u}} .
$$

This so-called Potthoff functional is the main result of the SEFA. It can be evaluated exactly given the reference system is solvable. The central approximation here is that the self-energy of the original system has been restricted, which means that the fully interacting model can at least be solved for a certain sub-space of self-energies. The variational principle can now be stated as:

$$
\frac{\delta \tilde{\Omega}_{\mathbf{t}, \mathbf{u}}\left[\boldsymbol{\Sigma}_{\mathbf{t}^{\prime}, \mathbf{U}}\right]}{\delta \Sigma_{\mathbf{t}^{\prime}, \mathbf{U}}}=0 \quad \text { or } \quad \frac{\partial \Omega\left(\mathbf{t}^{\prime}\right)}{\partial \mathbf{t}^{\prime}}=0
$$


where in the last formulation (2.11) is taken as a function of the variational parameters of the reference system. With this one now has to search for stationary points in the restricted domain of the self-energy, which is given by the choice of variational parameters in $t^{\prime}$. If the reference system is chosen reasonably well one can find a proper and controlled approximation for the grand potential (2.4) [Pot12a]. The SEFA yields this quantity in a thermodynamically consistent way, which means that it is evaluated exactly but for a restricted domain of the self-energy. Thus, one can use the derivatives of (2.11) at the stationary point for example with respect to the temperature or the chemical potential to find approximations for other thermodynamical quantities like energy or particle number [Pot12a].

Potthoff's self-energy functional (2.11) was the starting point to study various models for interacting fermions. Thereby, the choice of the reference system is of special importance. The interaction $\mathbf{U}$ has to be kept fixed while one is free to change the single-particle parameters in $t^{\prime}$, namely the hopping $t$ and the chemical potential $\mu$.

This also includes the freedom to leave out connections between sites. By setting certain hopping terms to zero one can construct reference systems which consist of separated clusters. Naturally, the self-energy and grand potential of such spatially tiled lattices can be computed using appropriate solvers. Thus, the use of cluster reference systems has proven to be a very successful way of applying the SEFA. Schemes that work along these lines are known as variational cluster approximations (VCA) [PAD03]. A very effective way to evaluate the self-energy functional (2.11) within the VCA is by applying the so-called Q-matrix formalism [Aic $\left.{ }^{+} 06 \mathrm{~b}\right]$. We will also make use of this technique in chapter 3.

Furthermore, one can use reference systems where bath sites are added. These extra degrees of freedom are coupled via some 'hybridization' to the cluster sites. The interaction on these is zero and they are uncorrelated for the original system, meaning that the hybridization vanishes. They enlarge the Hilbert space but do not change $\mathbf{U}$ or the selfenergy. Such bath sites have been used successfully in applications of the VCA, for example for systems with first order phase transitions. Yet, they do not always improve the quality of the approximation [Pot03b; Pot12a].

One also has the freedom to add local variational parameters to $t^{\prime}$. These include Weiß fields, for example staggered magnetic terms to investigate antiferromagnetism in the Hubbard model [ $\mathrm{Dah}^{+} 04 ; \mathrm{Aic}^{+}$06a]. Such local fields can be used within the VCA to study different phases with broken symmetries and continuous phase transitions.

The SEFA is connected to other methods. For example, the dynamical mean-field theory (DMFT) [Geo ${ }^{+96}$ ] can be derived within this framework. One can show its equivalence to a VCA with a reference system that consists of a single site cluster coupled to infinitely many bath sites 
[Pot03b]. In the broad scheme presented by the SEFA, one also finds links to methods like the cluster perturbation theory or cluster DMFT [Pot12a].

Additionally to the already mentioned examples the VCA was used to study various models of interacting fermions $\left[\mathrm{Aic}^{+}\right.$04; PB07; BHP08; $\mathrm{Arr}^{+}$09; BP10; Len 16]. A formulation of the VCA for systems consisting of interacting bosonic particles has also been developed, for example the Bose-Hubbard model [KD06] or lattice bosons in the superfluid phase [AKL11].

Given the merits and the wide applicability of cluster approximations derived from the SEFA we reiterate that such an approach would be very useful to have for spin Heisenberg systems. Yet, a Baym-Kadanoff respectively Luttinger-Ward functional (2.3) which is the starting point of the scheme is not readily available. A priori, it is not clear how such a functional could be derived. The SEFA is built on the observation that the local interaction and the non-interacting part are separable for the Hamiltonian and in the Baym-Kadanoff functional. On the other hand, the Heisenberg model with its exchange $\mathbf{J}$ is strictly and non-locally interacting. It proves helpful to look into further developments of the SEFA.

Another class of systems which are not included in standard VCA formulations are those with non-local interactions, as the necessary separation of local and non-local parts in the Hamiltonian is not possible any more. Attempts to include such interactions in theories like the dynamical mean-field theory were based on scaling arguments [SS96; SS00], assumptions about the structure how the fluctuation spectrum generated by these non-local interactions enters the free-energy functional [GSF01] or the GW method [SK02]. Later, Tong proposed a so-called extended variational cluster approximation (EVCA) for fermionic models with non-local interactions [Ton05]. In this approach, a suitable LuttingerWard functional is formally constructed from a fermionic coherent-state representation and tools of functional analysis are used to establish a cluster approximation for such systems. In chapter 3, we will develop a similar scheme to derive a VCA for spin systems. But first, other possible approaches to the problem are discussed in the remaining sections of this chapter. 


\subsection{THE SPIN DIAGRAM TECHNIQUE}

As was already mentioned in the last section one way to derive the SEFA is by using perturbation theory with regards to the electron interactions. The two most important quantities within this scheme, the functional (2.3) by Luttinger and Ward respectively Baym and Kadanoff as well as the self-energy, can be defined by means of an expansion in Feynman diagrams [LW60; BK61]. Naturally, to set up this diagrammatic representation one needs Wick's theorem for the products and correlators of fermionic (or bosonic) operators [Wic50; NO88]. Furthermore, a linked cluster theorem is needed to write the grand potential as the series of all connected diagrams [BDD58; NO88].

These theorems are not directly given for a Heisenberg Hamiltonian, where the operators do satisfy non-standard commutation relations and an interacting part is not obviously separated. Yet, a spin diagram technique has been proposed by Vaks, Larkin and Pikin [VLP68b] where equivalents to Wick's and the linked cluster theorem hold. So it seemed therefore natural to investigate if one can find an analogue to the BaymKadanoff functional within this theory.

Though it turns out that such a quantity is not available in a perturbative way, one can at least define a self-energy on the basis of the diagram technique. During the development of the spin VCA in chapter 3 we will make use of this quantity. This section includes a short introduction to the spin diagrams and its relevant aspects regarding a spin VCA. It is based on the books by Izyumov et al. [IKOS74; IS88].

One starts with a system at inverse temperature $\beta$ described by the already discussed Heisenberg Hamiltonian with an external field:

$$
\begin{aligned}
\mathbf{H} & =\mathbf{H}_{0}+\mathbf{H}_{i n t} \\
\mathbf{H}_{0} & =h \sum_{i} \mathbf{S}_{i}^{z} \\
\mathbf{H}_{i n t} & =\sum_{i, j} J_{i j}\left[S_{i}^{z} \mathbf{S}_{j}^{z}+\frac{1}{2}\left(\mathbf{S}_{i}^{+} \mathbf{S}_{j}^{-}+\mathbf{S}_{i}^{-} \boldsymbol{S}_{j}^{+}\right)\right],
\end{aligned}
$$

with commutation relations for the spin operators:

$$
\left[\boldsymbol{S}_{i}^{z}, \boldsymbol{S}_{j}^{+}\right]=\delta_{i j} \boldsymbol{S}_{i}^{+},\left[\boldsymbol{S}_{i}^{z}, \boldsymbol{S}_{j}^{-}\right]=-\delta_{i j} \boldsymbol{S}_{i}^{-},\left[\boldsymbol{S}_{i}^{+}, \boldsymbol{S}_{j}^{-}\right]=\delta_{i j} S_{i}^{z} .
$$

In (2.13) $\mathbf{H}$ is explicitly split into the non-interacting magnetic field $\mathbf{H}_{0}$ and the exchange interaction $\mathrm{H}_{\text {int }}$. With this choice one can set up a scheme to derive a spin diagram technique. One has to note that here the Hamiltonian 2.13 has ferromagnetic exchange. There also exists a formulation of the theory for an antiferromagnetic model [PSS69]. For simplicity we will mainly discuss the ferromagnetic case in this introductory section.

In the Heisenberg picture with the Hamiltonian $\mathbf{H}$ from 2.13, the spin operators are temperature respectively imaginary time $\tau$-dependent:

$$
\tilde{S}_{i}^{\alpha}(\tau)=e^{\tau H} S_{i}^{\alpha} e^{-\tau H},
$$


where $\alpha$ represents,+- or $z$. As usual the imaginary time is given in the interval $0<\tau<\beta$. A temperature Green's function is defined as an average of time-ordered products of spin operators [IS88]:

$$
\left\langle\mathrm{T} \tilde{\boldsymbol{S}}_{1}^{\alpha_{1}}\left(\tau_{1}\right) \ldots \tilde{\boldsymbol{S}}_{n}^{\alpha_{n}}\left(\tau_{n}\right)\right\rangle=\frac{\left\langle\mathrm{TS}_{1}^{\alpha_{1}}\left(\tau_{1}\right) \ldots \boldsymbol{S}_{\mathrm{n}}^{\alpha_{n}}\left(\tau_{\mathfrak{n}}\right) \boldsymbol{\sigma}(\beta)\right\rangle_{0}}{\langle\boldsymbol{\sigma}(\beta)\rangle_{0}} .
$$

Here $T$ is a symbol for the time-ordering with increasing $\tau$ and the brackets denote the statistical averages with regards to $\mathrm{H}$ respectively $\mathrm{H}_{0}$. The temperature scattering matrix $\sigma$ is introduced as follows:

$$
\begin{aligned}
e^{-\tau \mathbf{H}} & =e^{-\tau \mathbf{H}_{0}} \boldsymbol{\sigma}(\tau) \\
\boldsymbol{\sigma}(\boldsymbol{\tau}) & =T \exp \left(-\int_{0}^{\tau} \boldsymbol{H}_{\text {int }}\left(\boldsymbol{\tau}^{\prime}\right) \mathrm{d} \boldsymbol{\tau}^{\prime}\right) .
\end{aligned}
$$

Finally, the operators from (2.16) and (2.17) are given in interaction representation using the non-interacting Hamiltonian from (2.13):

$$
\boldsymbol{S}_{i}^{\alpha}(\tau)=e^{\tau \mathbf{H}_{0}} \boldsymbol{S}_{i}^{\alpha} e^{-\tau \boldsymbol{H}_{0}}, \mathbf{H}_{i n t}(\tau)=e^{\tau \boldsymbol{H}_{0}} \boldsymbol{H}_{i n t} e^{-\tau \boldsymbol{H}_{0}} .
$$

The two relations (2.16) and (2.17) introduce an expansion of temperature Green functions like $\left\langle\mathrm{T} \tilde{\boldsymbol{S}}_{i}^{\alpha_{i}}\left(\tau_{i}\right) \tilde{\boldsymbol{S}}_{j}^{\alpha_{j}}\left(\tau_{j}\right)\right\rangle$ in powers of $\boldsymbol{H}_{i n t}$ respectively interaction $J_{i j}$. Thus, they can be calculated by computing the individual averages of T-products of the operators $S_{i}^{\alpha}$.

Up to now this resembles the usual formalism for fermionic or bosonic systems where the Wick theorem is used to decompose the perturbative corrections to combinations of simple two-particle terms [NO88]. This is not directly available for the $S_{i}^{\alpha}$, but a similar scheme is used within the spin diagrammatic approach [VLP68b; IS88]. The basic idea is to commute operators $\boldsymbol{S}^{-}$out of any product in the expansion of (2.16) [IKOS74]:

$$
\begin{aligned}
& \left\langle\mathrm{T}\left\{\mathbf{S}_{1}^{\alpha_{1}}\left(\tau_{1}\right) \ldots \mathbf{S}_{\mu}^{-}(\tau) \ldots S_{\mathrm{n}}^{\alpha_{\mathfrak{n}}}\left(\tau_{\mathrm{n}}\right)\right\}\right\rangle_{0} \\
& =\Pi_{\mu 1}^{0}\left(\tau-\tau_{1}\right)\left\langle\mathrm{T}\left\{\left[\boldsymbol{S}_{1}^{\alpha_{1}}, \boldsymbol{S}_{\mu}^{-}\right]_{\tau_{1}} \boldsymbol{S}_{2}^{\alpha_{2}}\left(\tau_{2}\right) \ldots \boldsymbol{S}_{n}^{\alpha_{n}}\left(\tau_{\mathfrak{n}}\right)\right\}\right\rangle_{0} \\
& +\Pi_{\mu 2}^{0}\left(\tau-\tau_{2}\right)\left\langle\mathrm{T}\left\{\boldsymbol{S}_{1}^{\alpha_{1}}\left(\tau_{1}\right)\left[S_{2}^{\alpha_{2}}, \boldsymbol{S}_{\mu}^{-}\right]_{\tau_{2}} \ldots S_{n}^{\alpha_{n}}\left(\tau_{n}\right)\right\}\right\rangle_{0}+\ldots \\
& \ldots+\Pi_{\mu n}^{0}\left(\tau-\tau_{n}\right)\left\langle T\left\{S_{1}^{\alpha_{1}}\left(\tau_{1}\right) S_{2}^{\alpha_{2}}\left(\tau_{2}\right) \ldots\left[S_{n}^{\alpha_{n}}, S_{\mu}^{-}\right]_{\tau_{n}}\right\}\right\rangle_{0} \text {, }
\end{aligned}
$$

where the non-interacting transversal propagator is introduced with $y_{0}=$ $\beta h$ :

$$
\begin{aligned}
\Pi_{11^{\prime}}^{0}\left(\tau-\tau^{\prime}\right) & =\delta_{11^{\prime}} \frac{\left\langle\mathrm{TS}^{-}(\tau) \mathbf{S}^{+}\left(\tau^{\prime}\right)\right\rangle_{0}}{\left\langle\mathbf{S}^{z}\right\rangle_{0}} \\
& =e^{\frac{y_{0}}{\beta}\left(\tau-\tau^{\prime}\right)} \cdot\left\{\begin{array}{c}
1 /\left(e^{y_{0}}-1\right), \tau>\tau^{\prime} \\
e^{y_{0}} /\left(e^{y_{0}}-1\right), \tau<\tau^{\prime}
\end{array} .\right.
\end{aligned}
$$

Using the commutation relations (2.14) one can see that this procedure leads to a sum of averages with one spin operator less. If (2.19) is successively utilized until all $\mathbf{S}^{-}$and $\mathbf{S}^{+}$are eliminated, one ends up with 
products of $\Pi^{0}$ propagators and averages like $\left\langle\mathbf{S}_{1}^{z} \mathbf{S}_{2}^{z} \ldots \boldsymbol{S}_{\mathfrak{n}}^{z}\right\rangle_{0}$ which only include $\boldsymbol{S}^{z}$ operators.

These can be treated by an algorithm presented in [IS88]. Thereby, the averages break up into products of terms associated with a single site. They can be written as a cumulant expansion in the following form:

$$
\begin{aligned}
\left\langle\mathbf{S}_{1}^{z}\right\rangle_{0} & =\mathrm{SB}_{\mathrm{S}}\left(\mathrm{S} \mathrm{y}_{0}\right)=\mathrm{b}\left(\mathrm{y}_{0}\right) \\
\left\langle\mathbf{S}_{1}^{z} \mathbf{S}_{2}^{z}\right\rangle_{0} & =\mathrm{b}^{2}+\mathrm{b}^{\prime} \delta_{12} \\
\left\langle\mathbf{S}_{1}^{z} \mathbf{S}_{2}^{z} \mathbf{S}_{3}^{z}\right\rangle_{0} & =\mathrm{b}^{3}+\mathrm{b}^{\prime} \mathrm{b}\left(\delta_{12}+\delta_{13}+\delta_{23}\right)+\mathrm{b}^{\prime \prime} \delta_{12} \delta_{23},
\end{aligned}
$$

where $B_{s}$ is the Brillouin function for spin $S$ [IS88].

Hence, according to the relations given in (2.19) and (2.21) a temperature Green function of type (2.16) decomposes into the sum of all possible combinations of spin operators. Such a scheme can be called a Wick theorem for spin operators.

At this point one needs to introduce the rules for the diagram technique. Due to expansion (2.16) and (2.17) a Green function is written as an infinite series of averages of spin operators. Each individual average breaks up into all possible products given by the process above. They consist of propagation and interaction terms which correspond to the following basic elements of the diagrams:

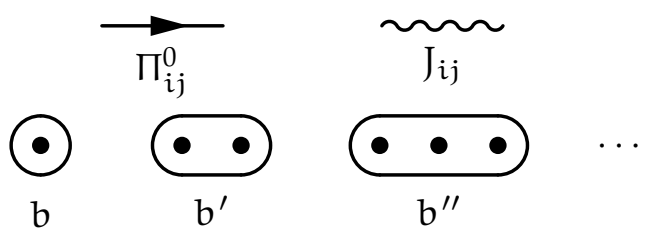

With these graphical representations one can build the individual diagrams. Three examples are given in the following:

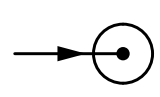

i)

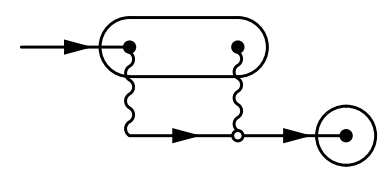

ii)

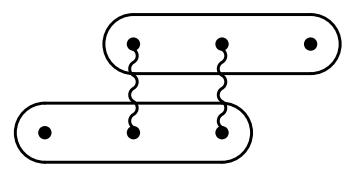

iii)

The first two diagrams belong to a two-spin Green function $\Pi^{+-}$, while iii) is part of the expansion of $\Pi^{z z}$. Several important features of the spin diagram formalism can be observed. Each transversal propagator, depicted as an arrow, ends in a so-called terminal part. The simplest is a circle which corresponds to $\left\langle\mathbf{S}_{i}^{z}\right\rangle_{0}$. An example is diagram i) representing the zeroth order in the expansion of $\Pi^{+-}$. Here, according to the scheme introduced in (2.19) and (2.20) we find $\left\langle\boldsymbol{S}_{i}^{-} \boldsymbol{S}_{j}^{+}\right\rangle_{0}=\Pi_{i j}^{0}\left\langle\boldsymbol{S}_{i}^{z}\right\rangle_{0}$. Further terminal parts can be seen in ii). For example, the first propagator ends in a vertex which belongs to a cumulant $b^{\prime}$. More complex terminal parts are possible and can be found in diagrams of higher order [IKOS74]. 
A vertex is represented as a starting respectively end point of a propagator arrow or by a dot within a cumulant. It is associated with a certain site and temperature. At a free vertex no interaction line enters. These depend on the expanded Green function, as can be seen by comparing ii) and iii). The former free vertices belong to a transversal propagator while the latter are part of a cumulant expansion of $\mathbf{S}^{\boldsymbol{z}}$ averages. In certain expansions also closed graphs appear which do not have any free vertex. Further details on the derivation of the spin diagrams can be found in the books by Izyumov et al. [IKOS74; IS88].

However, one important aspect of the expansion of Green functions (2.16) needs to be discussed. In the diagrammatic expansion of the numerator $\left\langle\mathrm{TS}_{1}^{\alpha_{1}}\left(\tau_{1}\right) \ldots S_{n}^{\alpha_{\mathfrak{n}}}\left(\tau_{\mathfrak{n}}\right) \boldsymbol{\sigma}(\beta)\right\rangle_{0}$ of (2.16) disconnected graphs appear. This means that they consist of several diagrams which are not connected by any propagator, interaction or cumulant. All but one of such sub-diagrams have no free vertices.

Yet, the expansion becomes less complex because a theorem on connectedness is valid for the technique [IKOS74; UFM63]. It follows that the average $\langle\boldsymbol{\sigma}(\beta)\rangle_{0}$ of the scattering matrix from (2.17) can be represented as the collection of all closed connected and disconnected diagrams. The graphs in the numerator of (2.16) can be ordered in such a way that the series $\langle\boldsymbol{\sigma}(\beta)\rangle_{0}$ in the denominator exactly cancels all diagrams without free vertices. Thus, the diagrammatic expansion of the temperature Green function from (2.16) itself is given by the series of connected graphs only with the corresponding number of free vertices [IKOS74].

Of special importance in the context of this thesis are the already mentioned two-spin Green functions:

$$
\begin{aligned}
\Pi_{i j}^{-+} & =\left\langle T S_{i}^{-}\left(\tau_{i}\right) S_{j}^{+}\left(\tau_{j}\right)\right\rangle, \\
\Pi_{i j}^{z z} & =\left\langle\mathrm{T}\left(\mathbf{S}_{\mathfrak{i}}^{z}\left(\tau_{i}\right)-\left\langle\mathbf{S}_{i}^{z}\right\rangle\right)\left(\mathbf{S}_{j}^{z}\left(\tau_{j}\right)-\left\langle\mathbf{S}_{j}^{z}\right\rangle\right)\right\rangle,
\end{aligned}
$$

where (2.22) is called transversal and (2.23) longitudinal. Naturally, both of them are temperature correlation functions. The fluctuations over the average values $\left\langle\boldsymbol{S}_{i}^{z}\right\rangle$ are used to define $\Pi_{i j}^{z z}$, which is needed to keep the diagrammatic series simple. The longitudinal correlation function (2.23) is called irreducible [IS88]. We will come back to the different possibilities defining $\Pi_{i j}^{z z}$ in chapter 3.5. 
The diagrammatic expansion of the transversal Green function (2.22) is given as:

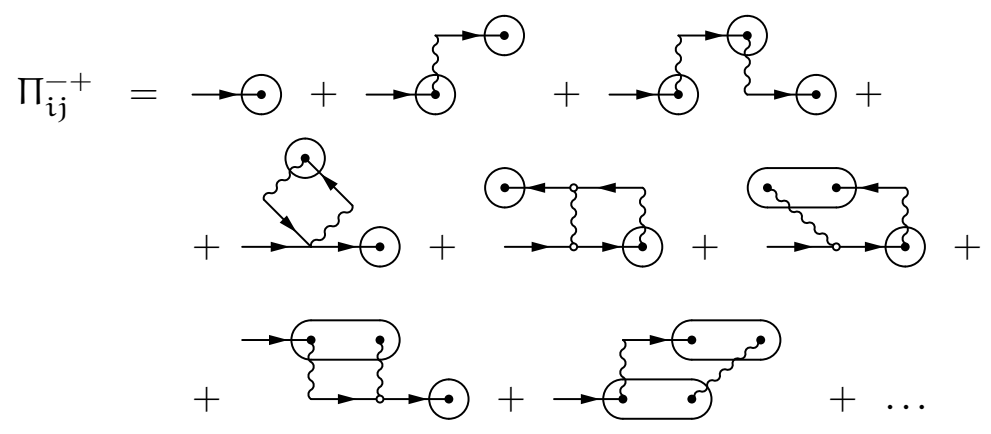

Equation (2.24) includes some but not all diagrams of second order in the interaction. This is also the case for the depiction of the diagrammatic series for (2.23):

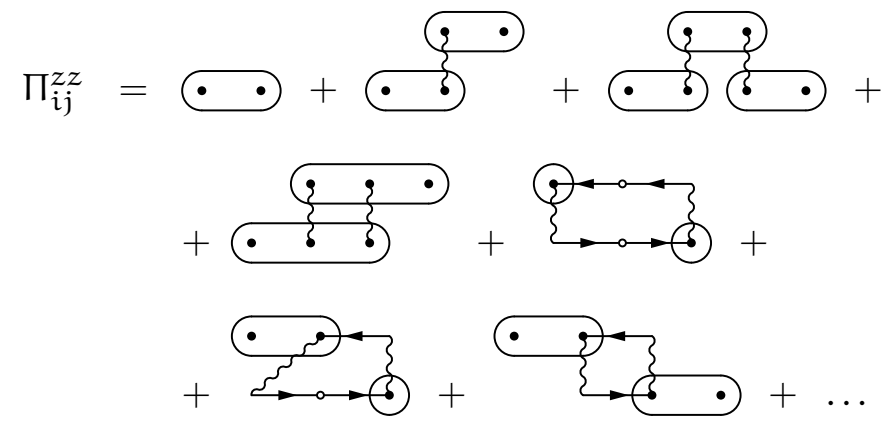

In standard perturbation theory one can construct the diagrammatic expansion of the Green function by using Dyson's integral equation and introducing the self-energy as the collection of diagrams which are irreducible with respect to one propagation line [ID89]. Such a construction can not be performed in the same way for the spin diagram technique. For the longitudinal correlation function (2.25) the notion of diagrams which are irreducible by cutting a propagation line is not sensible given the nature of the cumulants $b, b^{\prime}, b^{\prime \prime} \ldots$. In the case of the transversal one obstacle is given by the terminal part of the $\Pi^{0}$ propagator. In principle one can construct the expansion of $\Pi^{-+}$using an integral equation similar to the Dyson equation. Yet, it is more complex since one not only has to define a proper self-energy, but also a collection of diagrams which represent all possible terminal parts. Essentially, the notion of irreducibility with respect to propagation lines is not useful.

A different kind of integral equation has been proposed by Larkin et al. for the spin diagram technique [VLP68a]. Here, a self-energy is introduced which consists of all diagrams not separable by cutting along a single interaction line. So the transversal Green function (2.24) can be written using $\Sigma^{-+}$in the following way:

$$
\Pi^{-+}=\Sigma^{-+}+\Sigma^{-+} \mathrm{J} \Sigma^{-+}+\Sigma^{-+} \mathrm{J} \Sigma^{-+} \mathrm{J} \Sigma^{-+}+\ldots,
$$


where $\mathrm{J}$ is a line of exchange interaction. Except for the second and third on the right hand side of (2.24) all diagrams belong to the interaction irreducible transversal self-energy $\Sigma^{-+}$. Its zeroth order is given by $\rightarrow \odot$ . The series (2.26) can be represented by an integral equation:

$$
\Pi^{-+}=\Sigma^{-+}+\Sigma^{-+} \mathrm{J}^{-+} \text {. }
$$

One can also introduce a self-energy for $\Pi^{z z}$ as the collection of all diagrams from the series (2.25) who are not separable by cutting one interaction line. Here, $\bullet$ represents the zeroth order of $\Sigma^{z z}$. Subsequently, the longitudinal Green function can be constructed via the integral equation:

$$
\Pi^{z z}=\Sigma^{z z}+\Sigma^{z z} \mathrm{~J} \Pi^{z z} .
$$

The two relations (2.27) and (2.28) are called Larkin's equations [IS88]. They can be rewritten as:

$$
\Pi^{\alpha}=\left[\left(\Sigma^{\alpha}\right)^{-1}-J\right]^{-1},
$$

where $\alpha$ is a compact notation for $z z$ and -+ .

Thus, the computation of the spin Green functions reduces to finding the irreducible self-energy. Certain subsets of diagrams of this quantity can be taken into account to apply approximations to the correlation functions [IS88]. In the context of this thesis our focus is not to carry out such a scheme and actually calculate specific diagrams. However, with the interaction irreducible self-energies we have quantities at hand which can be used for a variational cluster approximation akin to Potthoff's approach discussed in 2.1. Larkin's equations will be used in the derivation of the spin VCA in section 3.2.

A major reason to investigate the spin diagram technique was to possibly construct a Baym-Kadanoff or Luttinger-Ward functional for the free energy of the a Heisenberg system in a perturbative way in analogy to the formalism used for fermionic systems [Bay62]. This seemed reasonable since a linked cluster theorem holds in this diagrammatic approach [BDD58; IKOS74]. This allows to represent the free energy as the sum of all connected closed diagrams.

The partition function $Z$ of the system is given by:

$$
\begin{aligned}
\mathbf{Z} & =\operatorname{Tr}\left(e^{-\beta \boldsymbol{H}}\right)=\operatorname{Tr}\left(e^{-\beta \mathbf{H}_{0}} \boldsymbol{\sigma}(\boldsymbol{\beta})\right) \\
& =\operatorname{Tr}\left(e^{-\beta \mathbf{H}_{0}}\right)\langle\boldsymbol{\sigma}(\beta)\rangle_{0},
\end{aligned}
$$

where we again used the interaction matrix $\sigma$ introduced in (2.17). Subsequently, the free energy becomes:

$$
\begin{aligned}
\mathrm{F} & =-\beta \ln Z \\
& =\mathrm{F}_{0}-\beta^{-1} \ln \langle\boldsymbol{\sigma}(\beta)\rangle_{0} .
\end{aligned}
$$

Here, $F_{0}$ is the free energy of the unperturbed Hamiltonian $\mathbf{H}_{0}$. In (2.31) the average $\langle\boldsymbol{\sigma}(\beta)\rangle_{0}$ appears. We already mentioned that a theorem of 
connectedness holds for this quantity [IKOS74; UFM63]. The average consists of all closed connected and disconnected diagrams. This series can be constructed by the exponential of $\Lambda$, which is the collection of closed connected graphs only. So the theorem on connectedness can be stated as $\langle\boldsymbol{\sigma}(\beta)\rangle_{0}=\exp (\Lambda)$.

Hence, the correction to the unperturbed free energy in (2.31) is given by all closed and connected diagrams in a linked cluster expansion:

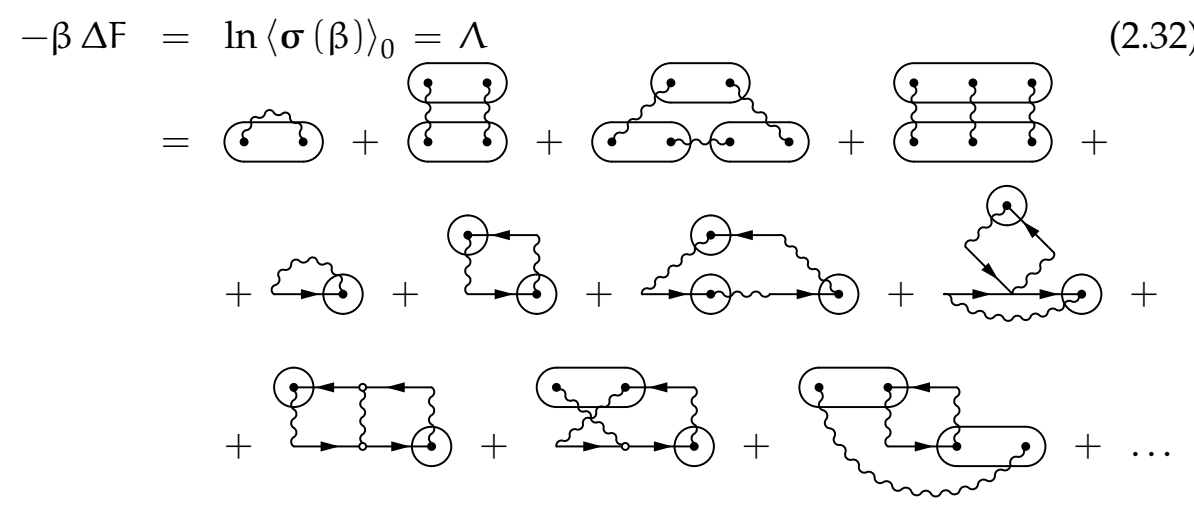

The depiction is given up to third order in the interaction, although this is not complete. The diagrammatic representation (2.32) of the free energy can be used to apply approximations by summation and subsequent computation of certain subsets of the diagrams [IKOS74]. In standard perturbation theory one can introduce the Baym-Kadanoff functional (2.3) using the linked cluster expansion of the grand potential [LW60]. Central to this is the Luttinger-Ward functional as the collection of all closed diagrams with full propagators inserted, so-called skeleton diagrams.

For the expansion (2.32) we could not find a similar scheme. This is due to the unusual structure of the spin diagram technique. As for the introduction of the self-energies of the Green functions, two features create problems - the cumulant elements (2.21) in the diagrams and the fact that transversal propagators are always associated with a terminal part. These are fundamental differences which do not allow to construct a functional following the perturbative scheme by Luttinger, Ward and Baym [LW60; Bay62]. As an example one can take the fourth diagram in the series (2.32). Such cumulant terms do not fit into the notion of a skeleton diagram. Yet, the fact that these are constituted of full propagators is decisive for the explicit independence of the Luttinger-Ward functional on the non-interacting Green function. As was discussed in section 2.1, this is in turn vital for setting up the SEFA [Pot12a]. Such a path is not available within the spin diagram technique. The structure of graphs including cumulant terms of more than two vertices prevents a viable definition of a full propagator skeleton expansion.

Moreover, the diagrammatic construction of a Baym-Kadanoff respectively Luttinger-Ward functional would have to be based on both Green functions. Further details on the interplay of the longitudinal and transversal correlation functions in the context of a generating functional 
method of the free energy can be found the work by Izyumov and Chashchin [IC01]. It supports our conclusion that one can not derive a suitable Luttinger-Ward functional by means of the diagram approach. Thus, for spin systems one needs to set up a different scheme, which is not based an a diagrammatic expansion.

Indeed, we will present such a formalism starting from a functionalintegral representation of the free energy in chapter 3. Yet, the Larkin self-energy (2.27)-(2.29) defined within spin perturbation theory plays a vital role in that approach. We can also make a second important observation. By comparing the free energy series (2.32) with the Green function expansions (2.24) and (2.25), one can see that the latter two are actually given by the derivative of $F$ with respect to the interaction $J$. This can be seen by bearing in mind that the derivative of a diagram in (2.32) is given by cutting one interaction line. We will deduce such differential relations in a compact and sound way in chapter 3.1 by using a path integral representation of the partition function respectively free energy.

In this section, we discussed the spin diagram technique for a ferromagnetic system. It is not possible to find a perturbatively defined Luttinger-Ward functional. Nevertheless, the Larkin self-energies to the transversal and longitudinal Green functions become important in the derivation of a spin VCA. Finally, we have to note that a formulation of the spin diagram formalism is also possible for antiferromagnetic as well as anisotropic Hamiltonians [PSS69; IKOS74] . Here, the expansions become more complex, yet one can always define suitable self-energies which are irreducible with respect to one interaction line, which is sufficient for the approach presented in this thesis. 


\subsection{OPERATOR TRANSFORMATIONS}

The standard SEFA discussed in chapter 2.1 is applicable for fermionic and also bosonic models. So one way to approach the problem of developing a variational cluster approximation for Heisenberg systems could be to make use of transformations of the spin operators. There are several schemes at hand where these are converted at least approximately into fermionic or bosonic degrees of freedom. With this it might be possible to apply the known VCA.

Two general difficulties arise though. First, such transformations usually lead to additional approximations or complications which would have consequences for a subsequent VCA. Second, the problem of nonlocal interactions remains for the new Hamiltonians, so the standard formalism can not be applied directly. This section discusses several possible transformations, especially with regard to their applicability in the present context.

\subsubsection{Fermionic Transformations}

We begin with an overview of fermionic transformations. As was already mentioned, to convert the Heisenberg models is usually only possible at the cost of new approximations or constraints. The one notable exception is the well-known Jordan-Wigner transformation that maps a spin-1/2 chain with first-neighbour interactions on a model of spinless fermions [JW28; Mat88]. The corresponding Heisenberg Hamiltonian is:

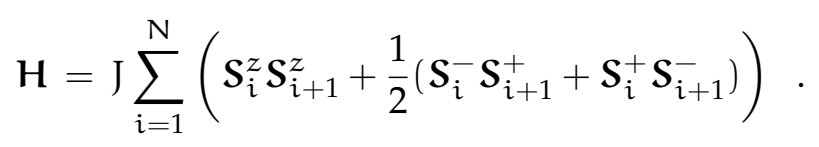

The spin-1/2 operators $\mathbf{S}^{ \pm}$follow fermionic anti-commutation relations on a given site:

$$
\left\{\mathbf{S}_{\mathbf{i}}^{+}, \mathbf{S}_{\mathbf{i}}^{-}\right\}=1,\left\{\mathbf{S}_{\mathbf{i}}^{+}, \mathbf{S}_{\mathbf{i}}^{+}\right\}=\left\{\mathbf{S}_{\mathbf{i}}^{-}, \mathbf{S}_{\mathbf{i}}^{-}\right\}=0,
$$

though on different sites commutation relations hold:

$$
\left[\mathbf{S}_{i}^{+}, \mathbf{S}_{j}^{-}\right]=\left[\mathbf{S}_{i}^{+}, \mathbf{S}_{j}^{+}\right]=\left[\mathbf{S}_{i}^{-}, \mathbf{S}_{j}^{-}\right]=0 .
$$

So, new operators are introduced:

$$
\begin{aligned}
& \mathbf{c}_{j}=\exp \left(i \pi \sum_{k=1}^{j-1} S_{k}^{+} S_{k}^{-}\right) S_{j}^{-}, \\
& \mathbf{c}_{j}^{\dagger}=\exp \left(-i \pi \sum_{k=1}^{j-1} S_{k}^{+} S_{k}^{-}\right) S_{j}^{+},
\end{aligned}
$$

with overall fermionic anti-commutation relations:

$$
\left\{\mathbf{c}_{i}, \mathbf{c}_{j}^{\dagger}\right\}=\delta_{i j}, \quad\left\{\mathbf{c}_{i}, \mathbf{c}_{j}\right\}=\left\{\mathbf{c}_{i}^{\dagger}, \mathbf{c}_{\mathbf{j}}^{\dagger}\right\}=0 .
$$


For these operators the following relations hold:

$$
S_{i}^{+} S_{i+1}^{-}=c_{i}^{\dagger} c_{i+1}, S_{i}^{z}=S_{i}^{+} S_{i}^{-}-\frac{1}{2}=c_{i}^{\dagger} c_{i}-\frac{1}{2} .
$$

Finally using the new fermionic operators the Hamiltonian (2.33) can be written as:

$$
H=J \sum_{i=1}^{N}\left[\frac{1}{2}\left(c_{i}^{\dagger} c_{i+1}+c_{i+1}^{\dagger} c_{i}\right)+\left(c_{i}^{\dagger} c_{i}-\frac{1}{2}\right)\left(c_{i+1}^{\dagger} c_{i+1}-\frac{1}{2}\right)\right] .
$$

This exact mapping shows that spins with $s=1 / 2$ and fermions are equivalent along a one-dimensional chain. This model can be solved exactly using Bethe-Ansatz [Bet31; Bax82], so there is no need to apply some cluster approximation. Yet, for the sake of the argument the Hamiltonian (2.39) could now in principle be used in a SEFA for this specific spin system. The problem for such an approach would be the non-local two-'particle' interaction. As was shown in 2.1 the standard VCA is only valid for a purely local interaction. For a system such as (2.39) one either has to apply some additional mean-field approximation to the Hamiltonian or resort to the EVCA proposed by Tong [Ton05]. This is a recurrent feature for the fermionic or bosonic transformations discussed in this section.

Before considering such an approach we need to see if the JordanWigner transformation can actually be applied in cases where a cluster approximation would be useful. Indeed, it has a limited applicability. In the original form introduced above the transformation is not valid for higher spins with a larger Hilbert space, which is only natural for a mapping on spinless fermionic degrees of freedom. Also, the present transformation (2.36) is only applicable in one spatial dimension.

Some work has been put into the extension of the Jordan-Wigner transformation to higher dimensions [Mat88; BO01]. At the heart of the problem lies the conversion from the spin commutation to the fermionic anticommutation relations. In (2.36) this was done through the exponential operators $\exp \left(i \pi S_{k}^{+} S_{k}^{-}\right)$, respectively the factors \pm 1 that arise in the particle basis of the Hilbert space. By virtue of these terms the transformation is highly non-local. The specific benefit of the one-dimensional model is that the spins are naturally ordered along the chain and that in the final fermionic Hamiltonian (2.39) the interactions have the same length as in the original spin model (2.33).

This is not given for Jordan-Wigner transformations in $\mathrm{D}>1$. A straight-forward extension to two-dimensional systems leads to fermionic Hamiltonians with highly non-local interactions [Azz93; Der01]. Additional approximations like a mean-field treatment of the operators are necessary in this case [Der $\left.{ }^{+} 03\right]$ unless one uses a special lattice geometry [CR04].

In a different approach an auxiliary gauge field coupled to the fermions is introduced which represents the phases found in (2.36) [Fra89; BO01; Der01]. The central problem of the higher dimensional Jordan-Wigner 
transformations remains though since these fields and correspondingly the particle interactions are highly non-local. The same holds for approaches in three or higher dimensions [HZ93; BO01]. Thus, to reasonably work with Jordan-Wigner transformations in dimensions $\mathrm{D}>1$ some additional approximations have to be applied. The general feature of highly non-local interactions is troublesome also for a possible application of a self-energy functional approach.

The Jordan-Wigner transformation has also been extended to Hamiltonians with spins with $s>1 / 2$ [BO01; Dob03]. Here, fermions of more than one type are introduced. To represent the spin Hilbert space certain constraints have to be imposed on these flavours of fermions to match the size $2 S+1$ of the Hilbert space of a spin Hamiltonian. Yet such local constraints can not be treated within the SEFA formalism.

This problem is akin to one which is faced with a different formalism. Fermionic Hamiltonians with local constraints can be treated using socalled X-operators. These were originally introduced by Hubbard to describe electronic systems in the atomic limit and have a certain range of applications in the theory of strongly correlated electrons [Hub65; OV04]. They are basically transition operators between states of a model. In principle one can express every Hamiltonian consisting of local operators which act on a finite and discrete Hilbert space by the $\mathrm{X}$-operators. This includes spin systems, either directly or after a fermionic transformation. For example, a spin-1 model mapped on two types of fermions can essentially be described by the local states $|0\rangle,|\uparrow\rangle$ and $|\downarrow\rangle$ since double occupation on a site is forbidden. Hence, the local constraint is directly taken into account when the Hamiltonian is written in terms of Hubbard transition operators using these three states.

Yet, like spins these $X$-operators are elements of a Lie algebra and exhibit non-standard commutation relations [OV04]. It was already discussed in chapter 1 that this is one of the main obstacles for the development of a SEFA for Heisenberg models. So they same holds true for Hamiltonians featuring $X$-operators unless they can be reduced to simple fermions or bosons. This means that nothing is won by transformations using transition operators. Systems with enforced local constraints on the Hilbert space pose equal problems regarding the development of a SEFA.

From the discussion in this section it is clear that the transformation of a spin Hamiltonian using fermionic operators usually leads to a model that does not allow a direct application of the self-energy functional approach. On two- or three-dimensional lattices long-ranged interactions appear. These models could only be utilized for a SEFA with additional approximations. Furthermore, transformed Hamiltonians with higher spins usually give no benefit over the original spin model. 


\subsubsection{Bosonic Transformations}

One of the problems associated with the fermionization of the spin operators was that it leads to additional non-locality in the Hamiltonian. To arrive at the correct anti-commutation relations one has to introduce unitary operators like in (2.37). These make the transformation highly non-local which in general is inherited by the Hamiltonian. Since bosons have commutation relations such terms need not to be introduced if the spins are transformed into bosonic degrees of freedom. In turn, additional non-localities are avoided which is a benefit in the present context.

A main disadvantage on the other hand lies in the structure of the Hilbert space of bosonic particles. For spin operators it is finite while for bosons it is not. So one in principle needs to introduce a certain constraint upon the transformed local Hamiltonian. An example of such a representation are hard-core bosons for spin-1/2 models where the Hilbert space is limited [MM56]. For Schwinger bosons the local constraints is realized by Lagrange multipliers [AA88a; AA88b]. As for the fermionic transformations discussed in the previous section such limitations can not be treated within the SEFA formalism.

A different kind of representation is used in spin wave theory. Here, excitations of a ferromagnetic or antiferromagnetic ground state are described by magnons, which are bosonic quasiparticles [FMH90]. Naturally, in the context of cluster approximations it is not reasonable to talk of spin wave excitations since they are inherently non-local. Yet one can use the formal transformation to find spin Hamiltonians that can possibly be treated within a variational cluster formalism.

We consider two well-known and related representations of the spins. The first one is the Holstein-Primakoff transformation for local operators with total spin quantum number $S$ [Kit87]:

$$
\mathbf{S}^{+}=\sqrt{2 \mathrm{~S}}\left(1-\frac{\mathbf{b}^{\dagger} \mathbf{b}}{2 \mathrm{~S}}\right)^{1 / 2} \mathbf{b}, \quad \mathbf{S}^{-}=\mathbf{b}^{\dagger} \sqrt{2 \mathrm{~S}}\left(1-\frac{\mathbf{b}^{\dagger} \mathbf{b}}{2 \mathrm{~S}}\right)^{1 / 2} .
$$

Given this representation of the spin ladder operators one finds for the $z$-component:

$$
\mathbf{S}^{z}=\mathrm{S}-\mathbf{b}^{\dagger} \mathbf{b} .
$$

The spin operators in (2.40) and (2.41) have the standard spin commutation relation $\left[\mathbf{S}^{+}, \mathbf{S}^{-}\right]=2 \mathbf{S}^{z}$ if the bosonic particles satisfy the canonical relations $\left[\mathbf{b}, \mathbf{b}^{\dagger}\right]=1$. Of course, operators acting on different sites commute.

The boson occupation number can be arbitrarily large. If one starts with the zero boson state which corresponds to $S^{z}=S$ then the number of bosons should not be bigger than $2 \mathrm{~S}$ since this part of the Hilbert space is non-physical. If $\boldsymbol{S}^{-}$acts on the state with exactly this occupation number respectively $\mathbf{S}^{z}=-\mathrm{S}$ the factor $\left(1-\frac{\mathbf{b}^{\dagger} \mathbf{b}}{2 \mathbf{S}}\right)^{1 / 2}$ gives zero. So the representation (2.40) has the reasonable feature that it disconnects the physical 
and the non-physical Hilbert space. One has to note, though, that matrix elements between non-physical states do not vanish.

The square roots of operators in (2.40) pose a problem for actual applications of this transformation. Usually one has to resort to expansions of these terms which is reasonable for low temperatures and subsequently only few excitations of the ground state [FMH90]. This additional approximation can be avoided by the second representation, the DysonMaleev transformation [Mat88]:

$$
\mathbf{S}^{+}=\sqrt{2 \mathrm{~S}}\left(1-\frac{\mathbf{b}^{\dagger} \mathbf{b}}{2 \mathrm{~S}}\right) \mathbf{b}, \quad \mathbf{S}^{-}=\sqrt{2 \mathrm{~S}} \mathbf{b}^{\dagger}
$$

These operators still lead to the $\boldsymbol{S}^{z}$ from (2.41) and satisfy the right commutation relations. They also disconnect the non-physical Hilbert space in the same way. Contrary to the Holstein-Primakoff transformation no expansion of square roots is needed, but this comes at a cost. The operators $\mathbf{S}^{+}$and $\mathbf{S}^{+}$are not conjugate any more and so the transformed Heisenberg Hamiltonian is non-hermitian.

The applicability of these two transformations for a spin VCA has been tested. Since the corresponding Hamiltonians feature bosonic operators the formulation for bosons is needed [KD06]. Furthermore, the interactions are non-local, so the extended variational cluster approximation has to be taken into account [Ton05]. Since no further expansion of operators is needed, the Dyson-Maleev transformation initially seemed to be the candidate with higher expectations. Yet, the non-hermitian Hamiltonian proves to be a severe problem. We find imaginary excitations in the spectrum of the systems Green function and the traces in the extended Potthoff self-energy functional can not be carried out properly. So an evaluation of the approximate free energy is not possible.

For the Holstein-Primakoff transformation we expand the square roots in (2.41) up to first order in $\frac{\mathbf{b}^{\dagger} \mathbf{b}}{2 \mathrm{~S}}$ :

$$
\mathbf{S}^{+} \approx \sqrt{2 S}\left(\mathbf{b}-\frac{\mathbf{b}^{\dagger} \mathbf{b} \mathbf{b}}{4 S}\right), \quad \mathbf{S}^{-} \approx \sqrt{2 \mathrm{~S}}\left(\mathbf{b}^{\dagger}-\frac{\mathbf{b}^{\dagger} \mathbf{b}^{\dagger} \mathbf{b}}{2 \mathrm{~S}}\right)
$$

An isotropic Heisenberg Hamiltonian (1.11) up to a constant approximately becomes:

$$
\begin{aligned}
\mathbf{H}=\sum_{i j} J_{i j}[ & S\left(\mathbf{b}_{i}^{\dagger} \mathbf{b}_{j}+\mathbf{b}_{j}^{\dagger} \mathbf{b}_{i}+2 \mathbf{b}_{i}^{\dagger} \mathbf{b}_{i}\right)+\mathbf{b}_{i}^{\dagger} \mathbf{b}_{i} \mathbf{b}_{j}^{\dagger} \mathbf{b}_{j} \\
& -\frac{1}{4} \mathbf{b}_{j}^{\dagger} \mathbf{b}_{i} \mathbf{b}_{j}^{\dagger} \mathbf{b}_{j}-\frac{1}{4} \mathbf{b}_{i}^{\dagger} \mathbf{b}_{j} \mathbf{b}_{i}^{\dagger} \mathbf{b}_{i} \\
& \left.-\frac{1}{4} \mathbf{b}_{i}^{\dagger} \mathbf{b}_{i} \mathbf{b}_{j}^{\dagger} \mathbf{b}_{i}-\frac{1}{4} \mathbf{b}_{j}^{\dagger} \mathbf{b}_{j} \mathbf{b}_{i}^{\dagger} \mathbf{b}_{j}\right]
\end{aligned}
$$

where we kept only the quartic terms. This again is a reasonable approximation for few excitations.

In the first line of the Hamiltonian (2.44) one-particle terms appear as well as a non-local interaction. The last lines feature so-called correlated 
hopping terms. Both of these interactions are not featured in the standard SEFA but have been treated in the EVCA [Ton05]. So in principle one could use this approach for the transformed spin Hamiltonian.

Though, one has to take care of the non-physical Hilbert space. No local state should have more than $2 \mathrm{~S}$ particles, otherwise one starts to accumulate errors. As before, additional constraints on the Hilbert space should not be enforced. We tested the transformation (2.44) within a bosonic EVCA for the simple ferromagnetic spin-1/2 Heisenberg chain with nearest-neighbour interaction. This means that maximally one particle is allowed per site. Thus, only the physical Hilbert space has to be taken into account if the number of bosons in a system is restricted to zero or one. This can be used for the cluster approximation. Here we can set the limit of one particle per cluster without entering the non-physical space.

Hence, the only reference system without any further approximations consists of single-site clusters. For a larger tiling of the lattice one would either enter the non-physical Hilbert space if more bosons are allowed or some physical states would be neglected if the limit is upheld. Any way, additional imprecision should be expected.

Our testing case for the bosonic spin EVCA used one- and two site clusters, the latter with maximally one or two particles per cluster. The exchange interaction J was taken to be the variational parameter. Though, the results were not encouraging. No reasonable stationary points of the self-energy functional were found. Moreover, in some cases it could not be evaluated properly. These kind of problems can in principle be encountered in every bosonic VCA approach [KD06]. They also appear within the EVCA. This point will be discussed in a different context in chapter 3.4.

The fact that the testing computations do not lead to proper results should not come as a surprise. First, the Hamiltonian (2.44) itself is subject to approximations. Second, one either has to use one-site clusters, which are probably too small to expect a meaningful reference system, or deal with the non-physical Hilbert space of the operators. And last, the parallel appearance of both non-local particle density interactions and correlated hopping in (2.44) might be too complicated for a sensible EVCA scheme. The fact that the functional can not be evaluated in many cases hints in that direction.

We will not go into additional details here or further pursue the endeavour of a spin VCA with bosonized Hamiltonian. The test cases have shown the severe problems associated with such an approach.

Thus, given the discussion in this section it becomes clear that also bosonic operator transformations of the spins do not lead to a successful VCA for Heisenberg models. The most promising representations come from spin wave theory. Yet, they suffer from non-hermitian Hamiltonians or additional approximations. Most of all, the non-physical Hilbert space of the bosonic operators poses a severe obstacle. 


\subsection{THE RESOLVENT SEFA}

This chapter will be concluded by an introduction of quite a different approach to the problem of developing a VCA for spin systems. Parts of the discussion in this section have been previously published in [FP10]. The approach is based on a resolvent technique devised for Hamiltonians involving operators which do not obey the standard boson or fermion algebra. It uses the full resolvent of the Hamiltonian and the partition function instead of the free energy. Among other models, the technique introduces a perturbative scheme for cases where the interaction part includes spin [KM84]. Thus, it is also applicable for Heisenberg systems of type (1.11).

The resolvent method starts with the observation that the partition function of a system with a Hamiltonian $\mathbf{H}$ and inverse temperature $\beta$ can be written as a contour integral:

$$
\mathbf{Z}=\operatorname{Tr} \exp (-\beta \mathbf{H})=\oint \frac{\mathrm{d} z}{2 \pi \mathrm{i}} \mathrm{e}^{-\beta z} \operatorname{Tr}(z-\mathbf{H})^{-1} .
$$

The Hamiltonian $\mathbf{H}=\mathbf{H}_{0}+\mathbf{H}_{\text {int }}$ is split up into an interacting part and some suitably chosen 'non-interacting' system. Note that we put the term in quotation marks as $\mathbf{H}_{0}$ can actually contain interactions. The only sensible requirement is that the problem defined by $\mathbf{H}_{0}$ should be easier to solve than the full Hamiltonian. The resolvent of $\mathbf{H}$ is given as:

$$
\mathbf{R}(z)=(z-\mathbf{H})^{-1}=\left(z-\mathbf{H}_{0}\right)^{-1} \sum_{n=0}^{\infty}\left(\frac{\mathbf{H}_{\text {int }}}{z-\mathbf{H}_{0}}\right)^{n},
$$

which can be expanded by means of the interacting part. The trace in (2.45) can be carried out over the complete set of eigenstates of $\mathbf{H}_{0}$. In this basis one can perturbatively define a 'generalized self-energy' operator $\Sigma(z)$ which depends on the variable of the contour integration [KM84]. With this the resolvent (2.46) and the partition function (2.45) becomes:

$$
\begin{aligned}
\mathbf{R}(z) & =\left(z-\mathbf{H}_{0}-\mathbf{\Sigma}(z)\right)^{-1} \\
\mathbf{Z} & =\oint \frac{\mathrm{d} z}{2 \pi i} e^{-\beta z} \operatorname{Tr}\left(z-\mathbf{H}_{0}-\mathbf{\Sigma}(z)\right)^{-1}
\end{aligned}
$$

Following Kuramoto [Kur83], the partition function (2.48) can be written as a functional of the resolvent $\mathbf{R}$ :

$$
\begin{aligned}
\mathrm{Z}[\mathbf{R}]-\mathrm{Z}_{0}=- & \beta \Phi[\mathbf{R}] \\
& +\beta \oint \frac{\mathrm{d} z}{2 \pi i} e^{-\beta z} \operatorname{Tr}\left[\left(z-\mathbf{H}_{0}\right) \mathbf{R}-\ln \left(\left(z-\mathbf{H}_{0}\right) \mathbf{R}\right)\right],
\end{aligned}
$$

where $Z_{0}$ is the partition function of the unperturbed Hamiltonian and:

$$
\Phi[\mathbf{R}]=\sum_{n} \frac{1}{n} \oint \frac{\mathrm{d} z}{2 \pi i} e^{-\beta z} \operatorname{Tr}\left(\Sigma^{(n)}[\mathbf{R}] \mathbf{R}\right) .
$$


Here, $\Sigma^{(n)}$ is the $n$-th order term in the expansion of the generalized self-energy. The functional $\Phi[\mathbf{R}]$ is defined as the collection of 'skeleton' diagrams. They are irreducible in the sense that they do not contain any self-energy insertions [Kur83]. It is the analogue to the Luttinger-Ward functional from standard diagrammatic perturbation theory. One can also show that the derivative of $\Phi[\mathbf{R}]$ from (2.50) with respect to the resolvent is given by the self-energy and that subsequently the partition function $Z[R]$ is stationary at the correct value of $\mathbf{R}$ [Kur83]:

$$
\begin{aligned}
& \frac{\delta \Phi[\mathbf{R}]}{\delta \mathbf{R}}=\frac{e^{-\beta z}}{2 \pi i} \mathbf{\Sigma}, \\
& \frac{\delta \mathbf{Z}[\mathbf{R}]}{\delta \mathbf{R}}=0 \rightarrow \mathbf{R}(z)=\left(z-\mathbf{H}_{0}-\mathbf{\Sigma}(z)\right)^{-1} .
\end{aligned}
$$

So (2.50) is similar to the Baym-Kadanoff functional discussed in chapter 2.1, using the resolvent instead of the Green function. The formulation of Kuramoto is carried out in an arbitrary basis of the Hamiltonian $\mathbf{H}_{0}$ and is independent on the nature of the operators involved. Thus, it is a promising starting point for the search of a variational cluster approximation for spin models.

We introduce the functional $F[\boldsymbol{\Sigma}]$ as the 'Legendre transform' of $\Phi[\mathbf{R}]$ :

$$
\mathrm{F}[\boldsymbol{\Sigma}]=\Phi[\mathbf{R}]-\oint \frac{\mathrm{d} z}{2 \pi i} e^{-\beta z} \operatorname{Tr}(\mathbf{R} \boldsymbol{\Sigma})
$$

to write $Z$ from (2.50) as a functional of the generalized self-energy:

$$
\mathrm{Z}[\boldsymbol{\Sigma}]-\mathrm{Z}_{0}=-\beta \mathrm{F}[\boldsymbol{\Sigma}]-\beta \oint \frac{\mathrm{d} z}{2 \pi \mathrm{i}} e^{-\beta z} \operatorname{Tr} \ln \left(\frac{z-\mathbf{H}_{0}}{z-\mathbf{H}_{0}-\boldsymbol{\Sigma}}\right) .
$$

The stationary point $\delta Z[\Sigma] / \delta \Sigma=0$ delivers the correct resolvent respectively self-energy.

Following Potthoff's idea we next consider a reference system $\mathbf{H}^{\prime}$ which has the same interaction part as $\mathbf{H}$, but a less complex and solvable $\mathbf{H}_{0}^{\prime}\left(p^{\prime}\right)$, with $p^{\prime}$ being some variational parameters.

$\Phi[\mathbf{R}]$ and subsequently $F[\Sigma]$ do not depend on the details of the noninteracting Hamiltonian [Kur83]. So we can eliminate the functional $F[\boldsymbol{\Sigma}]$ via combination of the partition functions $Z$ of $\mathbf{H}$ and $\mathbf{Z}^{\prime}$ of $\mathbf{H}^{\prime}$ :

$$
\begin{aligned}
Z[\Sigma]-Z_{0}=Z^{\prime} & -Z_{0}^{\prime}+\beta \oint \frac{d z}{2 \pi i} e^{-\beta z} \operatorname{Tr} \ln \left(\frac{z-\mathbf{H}_{0}^{\prime}}{z-\mathbf{H}_{0}^{\prime}-\Sigma^{\prime}}\right) \\
& -\beta \oint \frac{\mathrm{d} z}{2 \pi i} e^{-\beta z} \operatorname{Tr} \ln \left(\frac{z-\mathbf{H}_{0}}{z-\mathbf{H}_{0}-\boldsymbol{\Sigma}}\right) .
\end{aligned}
$$

As our central approximation we now set $\Sigma$ to be the self-energy of the reference system $\Sigma^{\prime}$, which basically means to restrict the interaction of the full system in a controlled way. By this we transform the partition function $Z$ (2.55) into a functional of $\Sigma^{\prime}$. For a given reference system 
$\mathbf{H}_{0}^{\prime}\left(\mathrm{p}^{\prime}\right)$ this approximate $\mathbf{Z}$ can be parametrized by the variational parameters $p^{\prime}$. If we use relation (2.47) for the resolvent it transforms to:

$$
\begin{aligned}
Z\left(p^{\prime}\right)=Z^{\prime} & -Z_{0}^{\prime}-Z_{0}-\beta \oint \frac{d z}{2 \pi i} e^{-\beta z} \operatorname{Tr} \ln \left(\frac{\mathbf{R}_{0}^{\prime}}{\mathbf{R}^{\prime}}\right) \\
& +\beta \oint \frac{\mathrm{d} z}{2 \pi i} e^{-\beta z} \operatorname{Tr} \ln \left(\left[\mathbf{R}^{\prime-1}+\mathbf{H}_{0}^{\prime}-\mathbf{H}_{0}\right] \cdot \mathbf{R}\right) .
\end{aligned}
$$

Besides $\mathbf{H}_{0}$ and subsequently $\mathbf{R}_{0}=\left(z-\mathbf{H}_{0}\right)^{-1}$ only quantities of the reference system appear in (2.56). The next step in our approach would be to determine a stationary point $\frac{\delta Z\left[\Sigma^{\prime}\right]}{\delta \Sigma^{\prime}}=0$ to find an approximate value for the partition function respectively free energy. Thus, the derivatives $\frac{\partial Z\left(p^{\prime}\right)}{\partial p^{\prime}}$ of (2.56) with respect to the parameters $p^{\prime}$ need to vanish.

The general scheme constructed here could at least in principle allow to generate systematic approximations for arbitrary spin systems. Following Potthoff's suggestion, one can use reference systems where the original lattice is split into several identical clusters with possible extra spins (external degrees of freedom) attached to them. By this procedure the Hilbert space is enlarged, so one would need to trace out the states of these external spins.

The second decision one has to take is how to break the Hamiltonian into a 'non-interacting' part and an interaction. Naturally, in the context of Heisenberg models of type 1.11 this is not obvious. In the case of an applied magnetic field one possibility is to treat this Zeeman term as the non-interacting system and the full exchange as the interaction. The at first sight rather intuitive idea turns out to be not very reasonable, since it leads to fully separated clusters. They are not embedded in any environment and there is no reason to believe that the results will improve direct cluster calculations. For any test computations carried out, the trivial solution that the magnetic field of the original model and the reference system are equal remains the only result of the variation. So indeed, this choice for the non-interacting part only leads to simple cluster Hamiltonians with the original parameters. Alternative and probably better ways to split $\mathbf{H}$ are to take the Ising part of 1.11 as $\mathbf{H}_{0}$ and the transversal terms to be $\mathbf{H}_{\text {int }}$ or vice versa. The magnetic field term should in any case also be in the non-interacting corner.

Subsequently, one would have to evaluate the free energy (2.56) for a chosen reference system. Thereby, the contour integral over $\mathrm{Tr} \ln$ of the given matrices has to be computed. This task can be boiled down to the poles and zeros of their eigenvalues, an applicable ansatz is found in [KD06]. One would have to either find the $z$-dependent eigenvalues directly or use the matrix identity $\operatorname{Tr} \ln M=\ln \operatorname{det} M$ to work with the determinant. The latter approach seems more feasible at first glance.

The huge obstacle for this approach and the evaluation of (2.56) comes with the size of the matrices involved. The resolvent technique works in the Hilbert space of the system. In the approximate partition function 
(2.56) the operators of the full lattice model $\mathbf{H}_{0}$ and $\mathbf{R}_{0}$ appear. Additionally, the resolvent and Hamiltonian of the reference system act in the same Hilbert space. Suppose $\mathbf{R}_{C}^{\prime}$ belongs to a single cluster, the full operator for the lattice of $\mathrm{N}$ such clusters would be:

$$
\begin{gathered}
\mathbf{R}^{\prime}=\mathbf{R}_{\mathrm{C}_{1}}^{\prime} \otimes \mathbb{1} \otimes \ldots \otimes \mathbb{1}+\mathbb{1} \otimes \mathbf{R}_{\mathrm{C}_{2}}^{\prime} \otimes \ldots \otimes \mathbb{1} \\
+\ldots+\mathbb{1} \otimes \mathbb{1} \otimes \ldots \otimes \mathbf{R}_{\mathrm{C}_{\mathrm{N}}} .
\end{gathered}
$$

Here we face a problem which similarly appears for the fermionic VCA. There, all Green function and self-energy matrices from the Potthoff functional act on the full lattice. The quantities of the reference system are block-diagonal with respect to a single cluster while the hopping matrix connects neighbouring clusters. Yet, the traces go over the spatial indices while in (2.56) they are carried out over the basis of the Hilbert space. For the standard VCA one can keep the computation manageable by performing a reduced Fourier transform in the meta-lattice of clusters [Sen08]. With this one can work on a single cluster rather than the full lattice at the cost of an additional k-vector summation.

Such a solution is not possible for the approach presented here. The Hilbert space is constituted of a product of single cluster Hamiltonian eigenstates and operators like (2.57), which would decompose in an appropriate manner. However, $\mathbf{H}_{0}$ and $\mathbf{R}_{0}$ from (2.56) generally do not. As was discussed above, any reasonable splitting of the Hamiltonian into a non-interacting part and an interaction involves terms with exchange between the sites. This means that the single cluster sub-spaces are connected by $\mathbf{H}_{0}$ and its resolvent. There is no way to get rid of these connections or to decompose the operators. So in contrast to standard VCA the last term in (2.56) has to be computed on the full lattice system respectively its Hilbert space. Since its rank increases exponentially with the number of sites, the evaluation of the partition function becomes impossible for any reasonable system size.

To summarize, in this section we have presented a variational approach for spin systems based on a resolvent technique. It is possible to formulate a variational principle for the partition function respectively free energy in the spirit of Potthoff. Yet its evaluation is not possible in a reasonable way. Depending on the choice of the non-interacting spin Hamiltonian, the result can be either trivial in that we only find an approximation where the lattice is simply divided in separate clusters, or basically impossible to carry out since the computations have to be performed in the Hilbert space of the full system. In both cases one has no gain by working with the resolvent approach. Unless one finds a way to appropriately decompose the Hilbert space, there is no reason to further pursue this approximation. 


\section{THE SPIN VARIATIONAL CLUSTER APPROXIMATION}

As discussed in the section 2.1, one needs to derive a Baym-Kadanoff respectively Luttinger-Ward functional to introduce a viable variational cluster approximation scheme for spin systems. This was not possible on the basis of the approaches presented in sections 2.2-2.4.

However, in the following chapter we will introduce an approach to establish a cluster approximation which starts from a path integral representation of the Heisenberg model. In the first two sections we will subsequently derive a suitable Luttinger-Ward functional. We will use this to introduce a free energy functional in section 3.3 which depends on the variational parameters of a cluster reference system. This finally leads to the introduction of the spin variational cluster approximation (SVCA).

We will also discuss in 3.4 the technical details of the evaluation of the free energy. The chapter concludes with a thorough discussion of certain important features of the novel method.

Parts of this chapter have been published in [FP14]. However, the derivations and discussions have been revised and expanded for this thesis. 


\subsection{THE FREE ENERGY FUNCTIONAL}

Originally, the self-energy functional approach was developed by Potthoff using the Baym-Kadanoff functional introduced within perturbation theory [Bay62; Pot03b; Pot03a]. The self-energy is defined in this framework as a series of one-particle irreducible diagrams and the Luttinger-Ward functional as the collection of all skeleton diagrams [LW60]. The Baym-Kadanoff functional is the connection between the static quantities - i.e. the grand-canonical potential - and the dynamical ones - Green functions and self-energies - and is found naturally in a diagrammatic formalism. Hence, the SEFA is well founded by using the concepts of perturbation theory, as was discussed in chapter 2.1.

But one can also derive the SEFA in a non-perturbative way. Potthoff pointed to general important properties which the Luttinger-Ward functional possesses [LW60; Pot06a]. They are shortly discussed in the following. Suppose we have a system of correlated electrons with a grandcanonical potential $\Omega$, the one-electron Green function $\mathbf{G}$ and the corresponding self-energy $\Sigma$. Then, as the first property, the Luttinger-Ward functional $\Phi=\tilde{\Phi}[\mathbf{G}]$ yields the grand canonical potential for the exact Green function:

$$
\Omega=\Phi+\operatorname{Tr} \ln \mathbf{G}-\operatorname{Tr} \mathbf{\Sigma} \mathbf{G},
$$

which leads to the Baym-Kadanoff functional. Furthermore, the derivative of the Luttinger-Ward functional with respect to the Green function gives the exact self-energy in the physical case. The Luttinger-Ward functional is also universal in the sense that the functional $\tilde{\Phi}[\mathbf{G}]$ is determined only by the interaction part of the Hamiltonian and not by the one-particle hopping. Therefore, the functional vanishes in the noninteracting case.

Potthoff then uses the functional-integral representation of the partition function and the path integral formulation of the Green function [NO88] to construct a Luttinger-Ward functional in a non-perturbative way [Pot06a]. The four properties outlined above can be verified for this functional. So the Luttinger-Ward functional can be derived without making use of the skeleton diagram expansion. Besides avoiding complications which can be associated with a perturbative approach [Pot06a], this construction has the merit that it makes approximations based on the Luttinger-Ward functional accessible for a larger class of systems.

Originally the SEFA was developed for electronic models with local interactions. As mentioned above, the interaction part defines the Luttinger-Ward functional and its independence on the hopping parameters is vital to establish the SEFA and its subsequent approximations (see chapter 2.1). To make use of this property the interacting part of the Hamiltonian needs to be unchanged for any kind of reference system used in the approach. These systems have to be solvable and incorporate 
some tiling of the original lattice. So, to keep the interaction unchanged it has to be purely local, otherwise there would be no suitable reference system. Therefore the original formulation of the SEFA did not allow the Hamiltonian to have non-local interactions. Yet, the derivation of the Luttinger-Ward functional within a functional-integral framework finally allows to mend this.

Ning-Hua Tong proposed an extension to the SEFA and the VCA for electronic systems with non-local interactions [Ton05]. In this work he uses the path-integral representation of two-particle correlation functions $\Pi$ in a similar way Potthoff uses the Green function in his construction of the Luttinger-Ward functional. This essentially leads to a new functional $\tilde{\Phi}[\mathbf{G}, \mathbf{\Pi}]$ which is the starting point for an Extended Variational Cluster Approximation (EVCA). In this approach the non-local interactions are treated conceptionally in the same way as the one-particle hopping terms. The new Luttinger-Ward functional is universal in the sense that it does not depend on either of these terms [Ton05]. While the EVCA certainly enhances the applicability of the Self-energy Functional Approach, there are some problems associated with the approximation. These will be discussed later in this chapter.

The path-integral construction of the Luttinger-Ward functional by Potthoff also provides a new approach for systems where a perturbative expansion fails to directly give a Baym-Kadanoff functional, as is the case for the quantum spin models treated in this thesis (see section 2.2). Given a suitable path-integral formulation for spin systems, one could in principle derive a Luttinger-Ward functional for the Heisenberg system and subsequently a Spin Variational Cluster Approximation (SVCA). This idea was also suggested by Tong [Ton05]. In the following, the approach will be worked out. First of all we need to establish a free energy functional based on a spin path-integral formalism. From there chapters 3.2 and 3.3 develop the spin Luttinger-Ward functional respectively the SVCA equations. This will be done using the notation introduced in the papers of Potthoff [Pot06a] and Tong [Ton05]. Finally, in section 3.4 a procedure is given how the SVCA equations can be evaluated.

As the starting point one needs a path-integral for quantum spins. It is well known that in the case of a system of bosonic or fermionic particles a path-integral can be introduced with the help of coherent states, which are eigenstates of the annihilation operators [NO88] [Per86]. For spin operators one can use analogously defined spin-coherent states. This concept was originally introduced by Radcliffe [Rad71] and later reviewed by Perelomov [Per86]. Appendix A introduces a version of spin-coherent states by Wiegmann [Wie88] and by Fradkin and Stone [FS88] which is then used to derive a path-integral representation for a spin model. 
Suppose we have an isotropic Heisenberg Hamiltonian with zero magnetic field on a lattice with sites denoted by $i$ and $j$ :

$$
\mathbf{H}=\sum_{i j} J_{i j} S_{i} S_{j}
$$

Using equations (A.27) and (A.30) from appendix A, one finds the corresponding partition function for a system with inverse temperature $\beta$ as:

$$
\begin{aligned}
Z & =\int \prod_{i} \mathcal{D} \vec{s}_{i} e^{-S\left(\vec{s}_{i}\right)} \\
S\left(\vec{s}_{i}\right) & =B\left(\vec{s}_{i}\right)+\int_{0}^{\beta} \mathrm{d} \tau \sum_{i j} J_{i j} \vec{s}_{i}(\tau) \vec{s}_{j}(\tau) .
\end{aligned}
$$

The variables $\vec{s}_{i}$ are vectors of length $S$ which represent the quasi-classical path of the spins $\mathbf{S}$. The function $\mathrm{B}\left(\vec{s}_{\mathfrak{i}}\right)$ only depends on the structure of the manifold spanned by the possible paths and incorporates topological effects of a spin system. It represents the Berry phase [Rad71] and does not include any information on the interaction $J_{i j}$, which is solely present in the second term of the action (3.3). The term $B\left(\vec{s}_{i}\right)$ is one reason why the explicit evaluation of the spin path integral is rather complicated. However, to derive expressions for the free energy of a certain model and subsequently equations that allow to establish a SVCA one only needs formal functional dependencies following from (3.3), i.e. , the precise form of $B$ is not important.

The temperature-dependent interaction is defined as $\mathrm{J}_{\mathfrak{i j}}\left(\tau-\tau^{\prime}\right):=$ $J_{i j} \delta\left(\tau-\tau^{\prime}\right)$. Since the exchange interaction will later serve as a variable for performing variations, an auxiliary field $\tilde{\mathrm{J}}$ is introduced, with the property $\tilde{\mathbf{J}}=\mathbf{J}$ for the exact physical system. The action is then formally written as a functional:

$$
\tilde{S}[\vec{s}, \tilde{\mathbf{J}}]=\mathrm{B}\left(\vec{s}_{i}\right)+\int_{0}^{\beta} \mathrm{d} \tau \int_{0}^{\beta} \mathrm{d} \tau^{\prime} \sum_{i j} \vec{s}_{\mathfrak{i}}(\tau) \tilde{J}_{i j}\left(\tau-\tau^{\prime}\right) \vec{s}_{j}\left(\tau^{\prime}\right) .
$$

Henceforth, functionals will be denoted by a tilde which will be omitted if the corresponding quantity assumes its physical value.

With this notation the partition function and free energy become:

$$
\begin{aligned}
& \tilde{Z}[\tilde{\mathbf{J}}]=\int \prod_{i} \mathcal{D} \vec{s}_{i} e^{-\tilde{S}[\vec{s}, \tilde{J}]}, \\
& \tilde{\mathrm{F}}[\tilde{\mathbf{J}}]=-\frac{1}{\beta} \ln \tilde{Z}[\tilde{\mathbf{J}}] .
\end{aligned}
$$

The form of the functional $\tilde{\mathrm{F}}[\tilde{\mathrm{J}}]$ depends on the structure of the exchange interaction $\tilde{\mathbf{J}}$ only, but not its specific value. With the help of the functional (3.5), the full spin-spin correlation function $\tilde{\Pi}$ is introduced as:

$$
\begin{aligned}
\tilde{\Pi}_{i j}\left(\tau-\tau^{\prime}\right) & =\left\langle T_{\tau} \vec{S}_{i}(\tau) \vec{S}_{j}\left(\tau^{\prime}\right)\right\rangle_{\tilde{S}}=-\beta \frac{\delta \tilde{F}[\tilde{\mathbf{J}}]}{\delta \tilde{\mathbf{J}}_{j i}\left(\tau-\tau^{\prime}\right)} \\
& =\frac{1}{\tilde{Z}} \int \prod_{i} \mathcal{D} \vec{S}_{i}\left(\vec{s}_{i}(\tau) \vec{s}_{j}\left(\tau^{\prime}\right) e^{-\tilde{S}[\vec{s}, \tilde{J}]}\right),
\end{aligned}
$$


where $\langle\ldots\rangle_{\tilde{S}}$ is the average with respect to the action given in 3.4.

In the more general case of an anisotropic Hamiltonian with a finite magnetic field:

$$
\begin{aligned}
\mathbf{H} & =\sum_{i} h \mathbf{S}_{i}^{z}+\sum_{i j}\left[J_{i j}^{z z} \mathbf{S}_{i}^{z} \mathbf{S}_{j}^{z}+\frac{1}{2} J_{i j}^{-+}\left(\mathbf{S}_{i}^{+} \mathbf{S}_{j}^{-}+\mathbf{S}_{i}^{-} \mathbf{S}_{j}^{+}\right)\right] \\
& =\sum_{i} h \mathbf{S}_{i}^{z}+\sum_{i j}\left[J_{i j}^{z z} \mathbf{S}_{i}^{z} \mathbf{S}_{j}^{z}+J_{i j}^{-+}\left(\mathbf{S}_{i}^{x} \mathbf{S}_{j}^{x}+\mathbf{S}_{i}^{y} \mathbf{S}_{j}^{y}\right)\right],
\end{aligned}
$$

one can introduce a similar functional-integral representation. Here, a path integral is again derived using the spin-coherent states [Per86]. This is done in complete analogy to the above isotropic case, as is shown in appendix A. In contrast to the $\mathrm{SU}(2)$-symmetric case one needs to define two fields $\tilde{\mathbf{J}}^{z z}$ and $\tilde{\mathbf{J}}^{-+}$to introduce the functional of the action (A.32):

$$
\begin{aligned}
& \tilde{S}\left[s^{\eta}, \tilde{\mathbf{J}}^{z z}, \tilde{\mathbf{J}}^{-+}\right]=\mathrm{B}\left(\mathrm{s}_{i}^{\eta}\right)+\int_{0}^{\beta} \mathrm{d} \tau \sum_{i} h s_{i}^{z}(\tau) \\
& +\int_{0}^{\beta} \mathrm{d} \tau \int_{0}^{\beta} \mathrm{d} \tau^{\prime} \sum_{i j} s_{i}^{z}(\tau)\left(\tilde{\mathbf{J}}^{z z}\right)_{i j}\left(\tau-\tau^{\prime}\right) s_{j}^{z}\left(\tau^{\prime}\right) \\
& +\int_{0}^{\beta} \mathrm{d} \tau \int_{0}^{\beta} \mathrm{d} \tau^{\prime} \sum_{i j}\left(s_{i}^{x}(\tau)\left(\tilde{\mathbf{J}}^{-+}\right)_{i j}\left(\tau-\tau^{\prime}\right) s_{j}^{x}\left(\tau^{\prime}\right)\right. \\
& \left.+s_{i}^{y}(\tau)\left(\tilde{\mathbf{J}}^{-+}\right)_{i j}\left(\tau-\tau^{\prime}\right) s_{j}^{y}\left(\tau^{\prime}\right)\right),
\end{aligned}
$$

along with the corresponding partition function $\tilde{Z}\left[\tilde{\mathbf{J}}^{z z}, \tilde{\mathbf{J}}^{-+}\right]$and free en$\operatorname{ergy} \tilde{\mathrm{F}}\left[\tilde{\mathbf{J}}^{z z}, \tilde{\mathbf{J}}^{-+}\right]$. In (3.8) the integrands are now explicitly dependent on the spin vector components $s^{\eta}$. As can be seen from the derivation of the path integral in appendix $A$, the Berry phase $B\left(\vec{s}_{i}\right)$ remains invariant [Per86; FS88]. This is expected since this topological term does not depend on a specific Hamiltonian but rather on the paths of the single spins along the sphere. This also justifies to include the Berry phase into the local part of the action $\tilde{S}_{\text {loc }}$ which is then constituted of the first two terms on the right hand side of (3.8).

The spin-spin correlation functions are introduced as:

$$
\begin{aligned}
\tilde{\Pi}_{i j}^{\xi \xi}\left(\tau-\tau^{\prime}\right) & =\frac{1}{\tilde{Z}} \int \prod_{i} \mathcal{D} s_{i}^{\eta}\left(s_{i}^{\xi}(\tau) s_{j}^{\xi}\left(\tau^{\prime}\right) e^{-\tilde{S}\left[s^{\eta}, \tilde{J}\right]}\right) \\
& =\left\langle T_{\tau} S_{i}^{\xi}(\tau) S_{j}^{\xi}\left(\tau^{\prime}\right)\right\rangle_{\tilde{S}},
\end{aligned}
$$

where the indices $\xi$ denote $z$, or + . From this we infer the following relations:

$$
\begin{aligned}
& \frac{\delta \tilde{\mathrm{F}}\left[\tilde{\mathbf{J}}^{z z}, \tilde{\mathbf{J}}^{-+}\right]}{\delta \tilde{\mathbf{J}}^{z z}}=-\frac{1}{\beta} \tilde{\Pi}^{z z} \\
& \frac{\delta \tilde{\mathrm{F}}\left[\tilde{\mathbf{J}}^{z z}, \tilde{\mathbf{J}}^{-+}\right]}{\delta \tilde{\mathbf{J}}^{-+}}=-\frac{1}{2 \beta}\left(\tilde{\Pi}^{-+}+\tilde{\Pi}^{+-}\right)
\end{aligned}
$$


which define the $\tilde{\Pi}^{\xi \xi}$ as functionals of the interactions $\tilde{\mathbf{J}}^{z z}$ and $\tilde{\mathbf{J}}^{-+}$. This functional-integral representation has to be used whenever the SU(2)symmetry is broken in the Hamiltonian, i.e. by a local magnetic field or an XZ-anisotropy. In the isotropic case this formulation naturally becomes equivalent to (3.6).

The definition (3.9) for the longitudinal $\Pi^{z z}$ is somewhat different from the standard one, which explicitly subtracts the expectation values of the spins and results in the connected correlation function:

$$
\left(\tilde{\boldsymbol{\Pi}}_{\mathrm{con}}^{z z}\right)_{i j}\left(\tau-\tau^{\prime}\right)=\left\langle\mathrm{T}_{\tau}\left(\mathbf{S}_{\mathbf{i}}^{z}(\tau)-\left\langle\mathbf{S}_{\mathbf{i}}^{z}\right\rangle\right)\left(\mathbf{S}_{\mathbf{j}}^{z}\left(\tau^{\prime}\right)-\left\langle\mathbf{S}_{j}^{z}\right\rangle\right)\right\rangle_{\tilde{S}^{c}},
$$

which can be introduced as the functional derivative of the free energy determined by an alternatively written action $\tilde{S}^{c}$. Here, Hartree - like terms are included in the local part. They would have to be determined self-consistently and read:

$$
\begin{aligned}
& \tilde{S}_{\text {loc }}^{c}\left[s^{\eta}\right]=\int_{0}^{\beta} \mathrm{d} \tau \sum_{i} h s_{i}^{z}(\tau) \\
& \quad+\int_{0}^{\beta} \mathrm{d} \tau \int_{0}^{\beta} \mathrm{d} \tau^{\prime} \sum_{i j}\left(J^{z z}\right)_{i j}\left(\tau-\tau^{\prime}\right)\left\langle s_{j}^{z}\right\rangle\left(2 s_{i}^{z}(\tau)-\left\langle s_{i}^{z}\right\rangle\right)
\end{aligned}
$$

In principle both the action $\tilde{S}^{c}$ as well as (3.8) can be used to derive the SVCA equations in the next chapters, with a varying local part and longitudinal correlation. Yet, the latter formulation leads to an additional set of constraints on local fields in the final formulation of the VCA for spin models since it will be mandatory that the local part remains unchanged. These constraints turn out out to be hard to satisfy for open spin systems, therefore $\tilde{S}^{c}$ will not be used in the evaluation in chapters 3.4 and 4 . However, this point will be discussed in detail in section 3.5.

In the following the functional relations (3.10) and (3.11) will be applied to derive the spin VCA equations. To keep the formulae simple, a compact notation without explicit reference to the components of the functions is used, where appropriate. Note that in this case any trace also involves a sum over the different longitudinal and transversal parts of the functions appearing. 
In the first step a Legendre transformed auxiliary functional $\tilde{A}$ is introduced utilizing the free energy functional $\tilde{F}$ from the previous section:

$$
\begin{aligned}
\tilde{A}\left[\tilde{\Pi}^{z z}, \tilde{\Pi}^{-+}, \tilde{\Pi}^{+-}\right]= & \tilde{\mathrm{F}}\left[\tilde{\mathbf{J}}^{z z}, \tilde{\mathbf{J}}^{-+}\right]-\operatorname{Tr}\left(\frac{\delta \tilde{\mathrm{F}}\left[\tilde{\mathbf{J}}^{z z}, \tilde{\mathbf{J}}^{-+}\right]}{\delta \tilde{\mathbf{J}}^{z z}} \tilde{\mathbf{J}}^{z z}\right) \\
& \quad-\operatorname{Tr}\left(\frac{\delta \tilde{\mathrm{F}}\left[\tilde{\mathbf{J}}^{z z}, \tilde{\mathbf{J}}^{-+}\right]}{\delta \tilde{\mathbf{J}}^{-+}} \tilde{\mathbf{J}}^{-+}\right) \\
= & \tilde{\mathrm{F}}\left[\tilde{\mathbf{J}}^{z z}, \tilde{\mathbf{J}}^{-+}\right]+\frac{1}{\beta} \operatorname{Tr}\left(\tilde{\Pi}^{z z} \tilde{\mathbf{J}}^{z z}\right) \\
& +\frac{1}{2 \beta} \operatorname{Tr}\left(\tilde{\Pi}^{+-} \tilde{\mathbf{J}}^{+-}\right)+\frac{1}{2 \beta} \operatorname{Tr}\left(\tilde{\Pi}^{-+} \tilde{\mathbf{J}}^{+-}\right)
\end{aligned}
$$

where the traces go over spatial indices and imaginary time respectively Matsubara frequencies. Here the functional relations (3.10) and (3.11) were used. It can be shown that the last two terms of (3.14) which contain the transversal correlation functions under the trace are equivalent. When a correlation function $C_{X Y}\left(\tau-\tau^{\prime}\right)=\left\langle\mathbf{X}(\tau) \mathbf{Y}\left(\tau^{\prime}\right)\right\rangle$ of operators $X$ and $Y$ which follow certain commutation relations is transformed to bosonic Matsubara frequencies, it holds that $C_{X Y}(\omega)=C_{Y X}(-\omega)$ [FW71]. This applies to the transversal spin correlation functions, so that $\Pi^{+-}(\omega)=\Pi^{-+}(-\omega)$. The traces in (3.14) include a summation over all bosonic Matsubara frequencies, so the two terms in the last row are equal for the physical quantities when one takes into account that $\mathrm{J}^{-+}$is constant in frequency space. It is reasonable to choose the corresponding functionals in such a way that these relations always hold. So $\tilde{A}$ becomes:

$$
\tilde{\mathrm{A}}\left[\tilde{\Pi}^{z}, \tilde{\Pi}^{\mathrm{t}}\right]=\tilde{\mathrm{F}}\left[\tilde{\mathbf{J}}^{z}, \tilde{\mathbf{J}}^{\mathrm{t}}\right]+\frac{1}{\beta} \operatorname{Tr}\left(\tilde{\Pi}^{z} \tilde{\mathbf{J}}^{z}\right)+\frac{1}{\beta} \operatorname{Tr}\left(\tilde{\Pi}^{\mathrm{t}} \tilde{\mathbf{J}}^{\mathrm{t}}\right) .
$$

For simplicity the longitudinal $(z z)$ and transversal $(-+)$ correlation functions are from now on denoted by a single $z$ and $t$, respectively. Equation (3.15) can be written in a compact notation:

$$
\tilde{\mathrm{A}}[\tilde{\Pi}]=\tilde{\mathrm{F}}[\tilde{\mathbf{J}}]+\frac{1}{\beta} \operatorname{Tr}(\tilde{\Pi} \tilde{\mathbf{J}})
$$

where the trace now includes the two spherical components.

The derivatives of the functional $\tilde{A}$ are:

$$
\begin{aligned}
& \frac{\delta \tilde{A}[\tilde{\Pi}]}{\delta \tilde{\Pi}^{z}}=\frac{1}{\beta} \tilde{\mathbf{J}}^{z}\left[\tilde{\Pi}^{z}, \tilde{\Pi}^{\mathrm{t}}\right], \\
& \frac{\delta \tilde{A}[\tilde{\Pi}]}{\delta \tilde{\Pi}^{t}}=\frac{1}{\beta} \tilde{\mathbf{J}}^{\mathrm{t}}\left[\tilde{\Pi}^{z}, \tilde{\Pi}^{\mathrm{t}}\right],
\end{aligned}
$$

which introduce the $\tilde{\mathbf{J}}^{\xi}$ as functionals of the $\tilde{\Pi}^{\xi}$. With the help of (3.16) we can write the free energy functional as Legendre transform of $\tilde{A}[\tilde{\Pi}]$ :

$$
\tilde{\mathrm{F}}[\tilde{\boldsymbol{\Pi}}]=\tilde{\AA}[\tilde{\boldsymbol{\Pi}}]-\frac{1}{\beta} \operatorname{Tr}(\tilde{\Pi} \tilde{\mathbf{J}})
$$


So far not much can be said about the properties of the auxiliary functional. Of course the goal is to eventually derive a Luttinger-Ward functional. To this end one needs to introduce the concept of a self energy for the correlation functions $\tilde{\Pi}$.

A similar quantity is used for example in the extended Dynamical Mean-Field Theory (EDMFT) for fermionic models with non-local interactions [SS96; SS99]. Here, a generalized self-energy $\Gamma$ can in principle be derived for a two-particle correlation function by using a cumulant expansion [SS00]. The resulting structure can be written in the general form [Ton05]

$$
\Gamma=\mathrm{J}+\alpha \Pi^{-1},
$$

with $\mathrm{J}$ being a matrix consisting of the interaction parameters of the model and $\alpha$ some constant introduced to control the analytical properties of the approach. A typical choice is $\alpha=1 / 2$, which is also used by Tong [Ton05]. However, in the case of spin models such a derivation does not readily exist, but one can nevertheless define a self-energy of the form (3.19) from analogy arguments [SS00; GSF01]. This definition for $\Gamma$ is sensible because it allows to introduce a Luttinger-Ward functional with respect to correlation functions which has the same structure as the standard functional for single-particle Green functions [Ton05; Pot06a].

On this level, the nature of the quantity $\Gamma$ seems somewhat artificial. Interestingly, it can be given a well-defined meaning using the spin diagram technique which was discussed in chapter 2.2. As was seen there one can formally resum the diagrams to find relations similar to Dyson's equation, which in the present context are called Larkin's equations [IS88]. They were given in (2.29) and can be written in matrix notation as :

$$
\Pi^{\xi}=\Sigma^{\xi}+\Sigma^{\xi} \mathbf{J}^{\xi} \Pi^{\xi}
$$

where again $\xi$ stands for $z$ or $t$ [IS88; PSS69]. The entries of the selfenergy matrix $\Sigma$ represent the collection of all diagrams in the expansion of the correlation function that are irreducible with respect to one interaction line. This is a conceptual difference to the diagrammatic definition of the usual self-energy and responsible for the slightly different structure of equation (3.20). It also means that Larkin's equation must not be identified with a Dyson equation of standard perturbation theory. A detailed discussion was given in 2.2. The important point is, one can formally rewrite (3.20) as:

$$
\left(\Sigma^{\xi}\right)^{-1}=\mathbf{J}^{\xi}+\left(\Pi^{\xi}\right)^{-1}
$$

Comparing this result to the expression (3.19), the previously defined quantity $\Gamma$ corresponds to the inverse of Larkin's self-energy with $\alpha=$ 1. As there is no need to provide an explicit expression for the Larkin self-energy in the present approach, (3.21) can be used as a suitable and reasonable spin self-energy. From now on $\Gamma^{\xi}$ will be conveniently used for the inverse Larkin self-energy $\left(\Sigma^{\xi}\right)^{-1}$. 
Two comments need to be added here. Firstly, it has to be pointed out that the above equations (3.21) hold for the single component correlation functions $\Pi^{\xi}$. As a consequence, a definition (3.19) for the correlation function $\Pi=\left\langle\vec{S}_{i} \vec{S}_{j}\right\rangle$ would be doubtful unless the system does have $\mathrm{SU}(2)$-symmetry. If that is not the case one can show that the inverse of a corresponding $\Gamma$ does not fulfill Larkin's criteria of being irreducible along one interaction line. So it would not have a reasonable meaning within this framework. Secondly, the longitudinal Larkin selfenergy was introduced for the connected correlation function (3.12) discussed at the end of section 3.1. So strictly speaking the interpretation of $\Gamma^{z}$ proposed here is valid only for this $\Pi_{\text {con }}^{z}$. The caveat will be discussed in section 3.5.

After introducing a reasonable concept of a self-energy one can now proceed with the derivation of a Luttinger-Ward-like functional for spin systems. Using Eqs. (3.17), (3.18) and the self-energies (3.21) the following functional derivatives are obtained:

$$
\begin{aligned}
\frac{\delta}{\delta \tilde{\Pi}^{z}}\left(\beta \tilde{\AA}[\tilde{\Pi}]+\operatorname{Tr} \ln \tilde{\Pi}^{z}\right) & =\tilde{\mathbf{J}}^{z}\left[\tilde{\Pi}^{z}, \tilde{\Pi}^{\mathrm{t}}\right]+\left(\tilde{\Pi}^{z}\right)^{-1} \\
& =\tilde{\Gamma^{z}}\left[\tilde{\Pi}^{z}, \tilde{\Pi}^{\mathrm{t}}\right] \\
\frac{\delta}{\delta \tilde{\Pi}^{\mathrm{t}}}\left(\beta \tilde{\mathrm{A}}[\tilde{\boldsymbol{\Pi}}]+\operatorname{Tr} \ln \tilde{\Pi}^{\mathrm{t}}\right) & =\tilde{\mathbf{J}}^{\mathrm{t}}\left[\tilde{\Pi}^{z}, \tilde{\Pi}^{\mathrm{t}}\right]+\left(\tilde{\Pi}^{\mathrm{t}}\right)^{-1} \\
& =\tilde{\Gamma}^{\mathrm{t}}\left[\tilde{\Pi}^{z}, \tilde{\Pi}^{\mathrm{t}}\right] .
\end{aligned}
$$

Finally, a generalized Luttinger-Ward functional can be defined as:

$$
\tilde{\Phi}[\tilde{\Pi}]=\beta \tilde{A}[\tilde{\Pi}]+\operatorname{Tr} \ln \tilde{\Pi} .
$$

Naturally one is faced with the question about the nature of this functional. The standard Luttinger-Ward functional can be derived as the collection of all connected closed skeleton diagrams [LW60]. A similar identification is not obvious for the formal definition (3.23), as the quantities appearing there are not all explicitly connected to a diagrammatic expansion. Even more so, as was discussed in chapter 2.2 it is not possible to find such a straight forward perturbative explanation. Yet, as Tong already pointed out for a fermionic system with non-local interactions [Ton05], a $\tilde{\Phi}$ such as (3.23) is closely related to the formal derivation of the Luttinger-Ward functional for the Hubbard model introduced by Potthoff [Pot06a]. Indeed one can show that several important properties which the standard Luttinger-Ward functional has are also satisfied by (3.23). These properties were already mentioned at the beginning of this chapter and are presented in detail in the paper from Potthoff [Pot06a]. They will be discussed with respect to the functional $\tilde{\Phi}$ defined in (3.23). 
According to the first property, the free energy of the system can be written as a functional of the correlation functions using $\tilde{\Phi}$. Equations (3.18) and (3.23) lead to:

$$
\begin{aligned}
\beta \tilde{\mathrm{F}}[\tilde{\boldsymbol{\Pi}}]= & \tilde{\Phi}[\tilde{\boldsymbol{\Pi}}]-\operatorname{Tr} \ln \tilde{\Pi}^{z}-\operatorname{Tr} \ln \tilde{\Pi}^{\mathrm{t}} \\
& -\operatorname{Tr}\left(\tilde{\Pi}^{z} \tilde{\mathbf{J}}^{z}\left[\tilde{\Pi}^{z}, \tilde{\Pi}^{\mathrm{t}}\right]+\tilde{\Pi}^{\mathrm{t}} \tilde{\mathbf{J}}^{\mathrm{t}}\left[\tilde{\Pi}^{z}, \tilde{\Pi}^{\mathrm{t}}\right]\right) .
\end{aligned}
$$

This functional can be seen as the Baym-Kadanoff functional for spin systems. This important result allows to develop SEFA- and VCA-like approximations.

Secondly, the functional derivatives of the Luttinger-Ward functional with respect to the correlation functions are given by the self-energies (3.21):

$$
\begin{aligned}
\frac{\delta \tilde{\Phi}[\tilde{\Pi}]}{\delta \tilde{\Pi}^{z}} & =\tilde{\Gamma}^{z}\left[\tilde{\Pi}^{z}, \tilde{\Pi}^{\mathrm{t}}\right] \\
\frac{\delta \tilde{\Phi}[\tilde{\Pi}]}{\delta \tilde{\Pi}^{\mathrm{t}}} & =\tilde{\Gamma}^{\mathrm{t}}\left[\tilde{\Pi}^{z}, \tilde{\Pi}^{\mathrm{t}}\right]
\end{aligned}
$$

This property is readily shown using Eqs. (3.22) and (3.23). It defines the self-energies as functionals of the two correlation functions. When evaluated at the physical values $\Pi^{\xi}$, the functionals $\tilde{\Gamma}^{\xi}$ acquire their physical value in Larkin's sense.

The third property the spin Luttinger-Ward functional should have is that it is universal in the sense that it does not explicitly depend on the interaction parameters J. This is however a direct consequence of the definition of the free energy functional $\tilde{\mathrm{F}}[\tilde{\mathrm{J}}]$ in (3.5) and (3.18). It is defined by the structure of the local Hamiltonian $\mathrm{H}_{\mathrm{loc}}$ and the way in which the $\tilde{\mathbf{J}}$ are introduced, not their explicit values. The property is inherited by the functional derivatives of $\tilde{\mathrm{F}}[\tilde{\mathrm{J}}]$ and the Legendre transform $\tilde{A}[\tilde{\Pi}]$. Therefore the functional (3.23) by construction does not depend on the specific interaction parameters.

The final property of the standard Luttinger-Ward functional that Potthoff pointed out is that it vanishes for $\mathrm{H}_{\text {loc }}=0$, which corresponds to $\mathrm{U}=0$ for the Hubbard model. This non-interacting limit leads to an easily solvable system of free fermions. Heisenberg models on the other hand remain complex for zero magnetic fields and the functional (3.23) consistently does not vanish in this case. This is a notable but expected difference to the usual Luttinger-Ward functional. 


\subsection{THE SPIN VCA EQUATIONS}

Even though the free energy functional $\tilde{\mathrm{F}}$ in equation (3.24) was derived in a formal way and is not directly based on a perturbative approach it can be used as starting point for approximations like the spin VCA. It is cast into a form with a structure which closely resembles the Baym-Kadanoff functional, and its constituent parts are well defined. The generalized Luttinger-Ward functional (3.23) has important postulated properties, and a self-energy can in principle be defined in a Larkin irreducible sense.

Following the idea of Potthoff's original approach and Tong's work, one now rewrites the free energy as a functional of the spin self-energies $\Gamma$ (3.21). For this step a Legendre transform of the Luttinger-Ward functional (3.23) is introduced:

$$
\begin{aligned}
\tilde{\mathrm{P}}[\tilde{\boldsymbol{\Gamma}}] & =\tilde{\Phi}[\tilde{\boldsymbol{\Pi}}]-\operatorname{Tr}\left(\frac{\delta \tilde{\Phi}\left[\tilde{\Pi}^{z}, \tilde{\Pi}^{\mathrm{t}}\right]}{\delta \tilde{\boldsymbol{\Pi}}^{z}} \tilde{\boldsymbol{\Pi}}^{z}\right)-\operatorname{Tr}\left(\frac{\delta \tilde{\Phi}\left[\tilde{\Pi}^{z}, \tilde{\Pi}^{\mathrm{t}}\right]}{\delta \tilde{\boldsymbol{\Pi}}^{\mathrm{t}}} \tilde{\boldsymbol{\Pi}}^{\mathrm{t}}\right) \\
& =\tilde{\Phi}[\tilde{\boldsymbol{\Pi}}]-\operatorname{Tr}\left(\tilde{\boldsymbol{\Gamma}}^{z} \tilde{\boldsymbol{\Pi}}^{z}\right)-\operatorname{Tr}\left(\tilde{\boldsymbol{\Gamma}}^{\mathrm{t}} \tilde{\boldsymbol{\Pi}}^{\mathrm{t}}\right)
\end{aligned}
$$

using Eqs. (3.25). The functional derivatives with respect to the selfenergies are:

$$
\begin{aligned}
\frac{\delta \tilde{P}[\tilde{\Gamma}]}{\delta \tilde{\Gamma}^{z}} & =-\tilde{\Pi}^{z}\left[\tilde{\Gamma}^{z}, \tilde{\Gamma}^{\mathrm{t}}\right], \\
\frac{\delta \tilde{P}[\tilde{\Gamma}]}{\delta \tilde{\Gamma}^{\mathrm{t}}} & =-\tilde{\Pi}^{\mathrm{t}}\left[\tilde{\Gamma}^{z}, \tilde{\Gamma}^{\mathrm{t}}\right] .
\end{aligned}
$$

These equations can be seen as defining $\tilde{\Pi}$ as a functional of $\tilde{\Gamma}$, i.e. one can write the free energy (3.24) with the help of (3.26) as a functional of these self-energies according to:

$$
\beta \tilde{\mathrm{F}}[\tilde{\Gamma}]=\tilde{\mathrm{P}}[\tilde{\Gamma}]-\operatorname{Tr} \ln \tilde{\Pi}-\operatorname{Tr}(\tilde{\Pi} \tilde{\mathbf{J}})+\operatorname{Tr}(\tilde{\Gamma} \tilde{\Pi})
$$

where all quantities are now to be taken as functionals of $\tilde{\Gamma}$. With the help of the defining relations for the self-energies (3.21) it is easy to see that the last two terms of (3.28) only give a constant contribution. This can be absorbed into $\tilde{\mathrm{P}}[\tilde{\Gamma}]$ and we end up with the expression:

$$
\beta \tilde{\mathrm{F}}[\tilde{\Gamma}]=\tilde{\mathrm{P}}[\tilde{\Gamma}]+\operatorname{Tr} \ln \left(\tilde{\Gamma}^{z}-\tilde{\mathbf{J}}^{z}\right)+\operatorname{Tr} \ln \left(\tilde{\Gamma}^{\mathrm{t}}-\tilde{\mathbf{J}}^{\mathrm{t}}\right),
$$

where again (3.21) was used.

A central feature of the fermionic Baym-Kadanoff functional not invoked yet is that it is stationary with respect to the corresponding physical one-particle Green function [BK61]. A similar stationary condition now holds for the functional (3.29) at the physical point $\tilde{\mathbf{J}}^{\xi}=\mathbf{J}^{\xi}$ :

$$
\beta \tilde{\mathrm{F}}_{\mathrm{SVCA}}[\tilde{\Gamma}]=\tilde{\mathrm{P}}[\tilde{\Gamma}]+\operatorname{Tr} \ln \left(\tilde{\Gamma}^{z}-\mathrm{J}^{z}\right)+\operatorname{Tr} \ln \left(\tilde{\Gamma}^{\mathrm{t}}-\mathrm{J}^{\mathrm{t}}\right) .
$$


In the following this $\tilde{\mathrm{F}}_{\mathrm{SVCA}}$ will be referred to as the spin VCA functional. The stationary principle can be written in the compact notation as:

$$
\begin{gathered}
\left.\frac{\delta \tilde{\mathrm{F}}_{\mathrm{SVCA}}[\tilde{\Gamma}]}{\delta \tilde{\Gamma}}\right|_{\tilde{\Gamma}=\Gamma}=0, \\
\tilde{\mathrm{F}}_{\mathrm{SVCA}}[\tilde{\Gamma}=\Gamma]=\mathrm{F} .
\end{gathered}
$$

The functional (3.30) is stationary for the exact self-energies and its value at this point gives the physical free energy.

In the last chapter the universality of the Luttinger-Ward functional (3.23) was discussed in the sense that it does not depend explicitly on the interaction functionals $\tilde{\mathrm{J}}$. This property is inherited by the Legendre transform $\tilde{P}$, which means that its functional form is the same for the free energy functionals (3.29) and (3.30). One can thus eliminate it by subtracting the two free energy functionals, to arrive at:

$$
\beta \tilde{F}_{S \vee C A}[\tilde{\Gamma}]=\beta \tilde{F}[\tilde{\Gamma}]+\operatorname{Tr} \ln \left(\frac{\tilde{\Gamma}^{z}-\mathbf{J}^{z}}{\tilde{\Gamma}^{z}-\tilde{\mathbf{J}}^{z}}\right)+\operatorname{Tr} \ln \left(\frac{\tilde{\Gamma}^{\mathrm{t}}-\mathbf{J}^{\mathrm{t}}}{\tilde{\Gamma}^{\mathrm{t}}-\tilde{\mathbf{J}}^{\mathrm{t}}}\right) .
$$

This functional $\tilde{F}_{S V C A}[\tilde{\Gamma}]$ is the equivalent of a Potthoff functional for the Heisenberg spin system. Although it was derived in a rather abstract way, it nevertheless can serve as the starting point for the SVCA.

To this end one has to choose a proper reference system, for example a system of exactly solvable clusters that shares some local Hamiltonian $\mathrm{H}_{\text {loc }}$ with the original system, while differing in the interactions J. The functionals $\tilde{\mathbf{J}}, \tilde{\Gamma}$ and $\tilde{\mathrm{F}}$ in (3.32) are then replaced by the corresponding quantities of this reference system, their particular values depending on the interactions $J_{c}$ of the cluster. With a specific choice of these parameters one restricts the space of spin self-energies and obtains the expression:

$$
\beta F_{S V C A}\left(J_{c}\right)=\beta F_{c}+\operatorname{Tr} \ln \left(\frac{\Gamma_{c}^{z}-J^{z}}{\Gamma_{c}^{z}-J_{c}^{z}}\right)+\operatorname{Tr} \ln \left(\frac{\Gamma_{c}^{t}-J^{t}}{\Gamma_{c}^{t}-J_{c}^{t}}\right) .
$$

Examples of suitable cluster systems for a square lattice can be found in figure 3.1, including possible variational parameters. Note that like in the fermionic or bosonic approaches one has a rather large freedom regarding the clusters and parameters [Pot06b]. The exchange interactions connecting different spins as well as the components $\mathrm{J}_{\mathcal{C}}^{z}$ and $\mathrm{J}_{\mathfrak{c}}^{\mathrm{t}}$ can in principle be varied independently from one another. Also, additional sites can be added to the boundary of the cluster, and so on. However, to keep the computation tractable, one usually restricts the variational space to a reasonable set of parameters. The results of the SVCA will of course depend on the specific choice of the reference system. In general, one will expect that the approximation becomes better and less dependent on the actual selection of parameters with increasing cluster size. Similar to the SEFA for fermions it is hard to define a limit where the method becomes exact. For the present theory this is the special case $\mathbf{J} \rightarrow 0$, i.e. a model of decoupled spins. Here, the SVCA is exact for a 


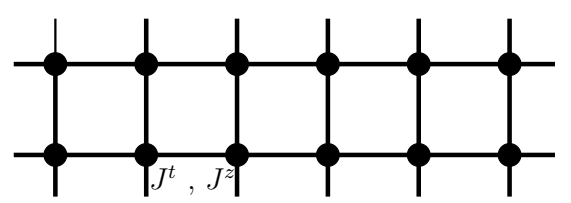

(a)

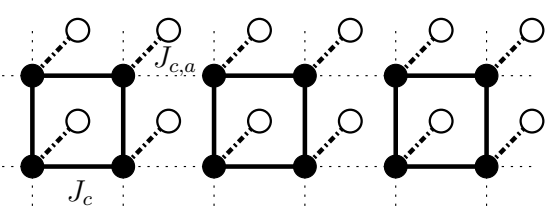

(c)

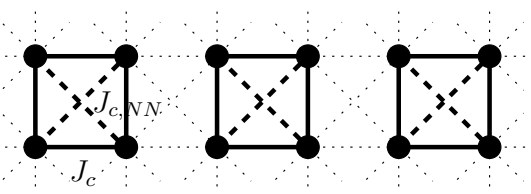

(e)

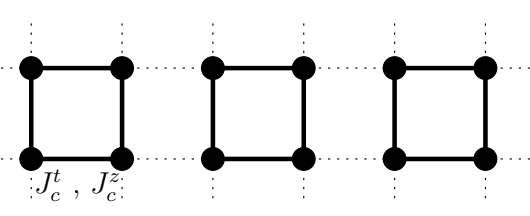

(b)

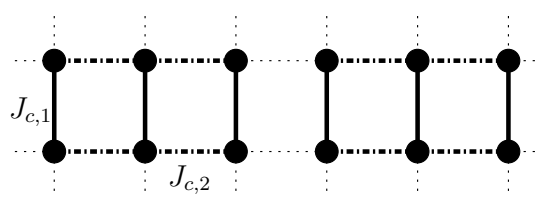

(d)

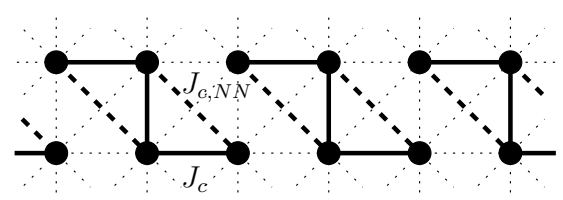

(f)

Figure 3.1: (a) The original square lattice with interactions $\mathrm{J}^{z}$ and $\mathrm{J}^{\mathrm{t}}$. (b) An example of a reference system used in the SVCA consisting of simple four-spin clusters with the possible variational parameters $\mathrm{J}_{\mathfrak{c}}^{z}$ and $J_{\mathfrak{c}}^{\mathrm{t}}$ which can be varied independently. (c) The same reference system as (b) but with additional auxiliary sites interacting through the variational parameter $J_{c, a} \cdot(d)$ A six-spin cluster system which demonstrates the possibility of two different parameters $J_{c, 1}$ and $J_{c, 2}$ connecting different sites. (e), (f) Two examples of clusters usable in the case of an additional next-nearest-neighbour interactions in the original Hamiltonian. Here the two parameters $\mathrm{J}_{\mathrm{c}}$ and $\mathrm{J}_{\mathrm{c}, \mathrm{NN}}$ can also be varied independently.

reference system of single-site clusters. Further discussion and details on the actual cluster systems which are used for a given model can be found in section 3.5.

Similar to the SEFA for fermionic systems, after one has chosen a proper reference system the task consists in finding a stationary point of the SVCA equation (3.33) with respect to the parameters $J_{c}$. An extremal point determined in this way represents an approximation to the stationary condition (3.31). 


\subsection{EVALUATION OF THE SPIN VCA EQUATIONS}

To determine the stationary points of the SVCA equation it has to be computed explicitly depending on the variational parameters. This section discusses the actual technical implementation of evaluating (3.33). As the starting point $\mathrm{F}_{\mathrm{S} \vee C A}\left(\mathrm{~J}_{\mathrm{c}}\right)$ it is rewritten as:

$$
\begin{aligned}
\beta F_{S V C A}\left(J_{c}\right)= & \beta F_{c}-\operatorname{Tr} \ln \left(\left(\Pi_{c}^{z}\right)^{-1}-V^{z}\right)^{-1}+\operatorname{Tr} \ln \Pi_{c}^{z} \\
& -\operatorname{Tr} \ln \left(\left(\Pi_{c}^{t}\right)^{-1}-V^{t}\right)^{-1}+\operatorname{Tr} \ln \Pi_{c}^{t} .
\end{aligned}
$$

Hereby $\left(\Pi_{\mathcal{c}}^{\xi}\right)^{-1}=\Gamma_{\mathfrak{c}}^{\xi}-J_{\mathfrak{c}}^{\xi}$ was obtained from equation (3.21) and the interaction matrix $\mathbf{V}^{\xi}:=\mathrm{J}^{\xi}-\mathrm{J}_{\mathbf{c}}^{\xi}$ was introduced. All quantities with the subscript $c$ belong to a chosen reference system where the original lattice of $\mathrm{N}$ sites is tiled into clusters of $\mathrm{N}_{\mathrm{c}}$ sites.

It is now advisable to Fourier transform the terms in (3.34) with respect to the meta lattice of the clusters [Sen08]. One ends up with a reduced wave vector representation in which the correlation functions $\Pi_{\mathcal{c}}^{\xi}$ are naturally diagonal. The interaction matrix $\mathbf{V}^{\xi}$ on the other hand is not and so will be dependent on a wave vector $k$. The traces in the SVCA free energy (3.34) will thus transform into sums over the cluster site indices and $k$. In variational cluster approaches the summation over the wave vectors can be approximated by a grid covering the reduced Brillouin zone where the number of terms in the sum is given by the number of clusters $\mathrm{N} / \mathrm{N}_{\mathrm{c}}$. More details on this formalism can be found in appendix C. In addition to the lattice indices the traces in (3.34) also include a sum over bosonic Matsubara frequencies $\omega_{n}=2 \pi n T$. To compute the SVCA free energy one has to provide a suitable and efficient way to carry out the different summations. Several strategies have been introduced for the variational cluster approaches [Pot03a; $\mathrm{Aic}^{+} 06 \mathrm{a}$; $\mathrm{Aic}^{+}$06b; KD06; Sen08].

Before the procedure used in the present work is introduced, one has to note that the contributions to (3.34) for the longitudinal respectively transversal correlation function are evaluated separately. So, the terms:

$$
\begin{aligned}
\mathrm{K}^{\xi}\left(\mathbf{J}_{\mathbf{c}}\right) & =-\operatorname{Tr} \ln \left(\left(\boldsymbol{\Pi}_{\mathbf{c}}^{\xi}\right)^{-1}-\mathbf{V}^{\xi}\right)^{-1}+\operatorname{Tr} \ln \boldsymbol{\Pi}_{\mathbf{c}}^{\xi} \\
& =\operatorname{Tr} \ln \left(\mathbf{1}-\mathbf{V}^{\xi} \boldsymbol{\Pi}_{\mathbf{c}}^{\xi}\right),
\end{aligned}
$$

are treated individually for $\xi=t$ and $\xi=z$. The discussion starts with the transversal part.

The Q-matrix formalism which was introduced for fermionic $\left[\mathrm{Zac}^{+} 02\right.$; $\mathrm{Aic}^{+}$06b] and bosonic systems [KD06] will be used in the following. The starting point in these approaches is the Lehmann representation of the corresponding Green function. Analogously, within the SVCA it will be the Lehmann representation of the spin correlation functions. The 
transversal $\Pi_{c}^{t}=\left\langle S_{i}^{-}(\tau) S_{j}^{+}\left(\tau^{\prime}\right)\right\rangle_{c}$ on the cluster can be written with Matsubara frequencies as:

$$
\begin{aligned}
\left(\Pi_{c}^{t}\right)_{i j}\left(\omega_{l}\right) & =\frac{1}{Z} \sum_{n, m} \frac{e^{-\beta E_{n}}-e^{-\beta E_{m}}}{\omega_{l}-\left(E_{n}-E_{m}\right)}\left\langle m\left|S_{i}^{-}\right| n\right\rangle\left\langle n\left|S_{j}^{+}\right| m\right\rangle \\
& =\frac{1}{Z} \sum_{n, m} \frac{e^{-\beta E_{n}}-e^{-\beta E_{m}}}{\omega_{l}-\left(E_{n}-E_{m}\right)}\left\langle n\left|S_{i}^{+}\right| m\right\rangle\left\langle n\left|S_{j}^{+}\right| m\right\rangle,
\end{aligned}
$$

where the vectors $|n\rangle$ are the eigenstates of the cluster system Hamiltonian and the $E_{n}$ the corresponding energies. This object can be analytically continued to establish a function of the complex variable $\omega$. To rewrite the correlation function using the Q-matrices one defines with the help of a multi-index $\alpha=(n, m)$ :

$$
\begin{aligned}
\mathbf{Q}_{\alpha i}^{\mathrm{t}} & =\sqrt{\frac{\mid e^{-\beta E_{n}}-e^{-\beta E_{m} \mid}}{Z}}\left\langle n\left|S_{i}^{+}\right| m\right\rangle, \\
\mathbf{g}_{\alpha \beta}^{\mathrm{t}} & =\delta_{\alpha \beta} \operatorname{sgn}\left(e^{-\beta E_{n}}-e^{-\beta E_{m}}\right), \\
\lambda_{\alpha \beta}^{t} & =\delta_{\alpha \beta}\left(E_{n}-E_{m}\right)=\lambda_{\alpha}^{t}, \\
\Lambda^{t}(\omega) & =\frac{g^{t}}{\omega-\lambda^{t}},
\end{aligned}
$$

where $\omega$ in the last line has to be understood as being multiplied by the unity matrix. It is important to note that a certain combination $\alpha$ is taken into account only when the correlation function has a non-vanishing pole at $\lambda_{\alpha}^{t}$. With the definitions in (3.37) one can now write:

$$
\Pi_{c}^{\mathrm{t}}(\omega)=\left(\mathbf{Q}^{\mathrm{t}}\right)^{+} \boldsymbol{\Lambda}^{\mathrm{t}}(\omega) \mathbf{Q}^{\mathrm{t}}=\left(\mathbf{Q}^{\mathrm{t}}\right)^{+} \frac{\mathbf{g}^{\mathrm{t}}}{\omega-\boldsymbol{\lambda}^{\mathrm{t}}} \mathbf{Q}^{\mathrm{t}}
$$

The other term inside the logarithm in (3.35) can also be rewritten with the above definitions as:

$$
\begin{aligned}
\left(\left(\Pi_{c}^{t}\right)^{-1}-\mathbf{V}^{t}\right)^{-1} & =\Pi_{c}^{t}\left(1-\mathbf{V}^{t} \Pi_{c}^{t}\right)^{-1}, \\
& =\left(\mathbf{Q}^{\mathfrak{t}}\right)^{+} \Lambda^{t} \mathbf{Q}^{\mathrm{t}}\left(\mathbf{1}-\mathbf{V}^{\mathrm{t}}\left(\mathbf{Q}^{\mathrm{t}}\right)^{+} \Lambda^{\mathrm{t}} \mathbf{Q}^{\mathrm{t}}\right)^{-1}, \\
& =\left(\mathbf{Q}^{\mathrm{t}}\right)^{+} \boldsymbol{\Lambda}^{\mathrm{t}}\left(\mathbf{1}-\mathbf{Q}^{\mathrm{t}} \mathbf{V}^{\mathrm{t}}\left(\mathbf{Q}^{\mathrm{t}}\right)^{+} \boldsymbol{\Lambda}^{\mathrm{t}}\right)^{-1} \mathbf{Q}^{\mathrm{t}}, \\
& =\left(\mathbf{Q}^{\mathrm{t}}\right)^{+}\left(\left(\boldsymbol{\Lambda}^{\mathrm{t}}\right)^{-1}-\mathbf{Q}^{\mathrm{t}} \mathbf{V}^{\mathrm{t}}\left(\mathbf{Q}^{\mathrm{t}}\right)^{+}\right)^{-1} \mathbf{Q}^{\mathrm{t}}, \\
& =\left(\mathbf{Q}^{\mathrm{t}}\right)^{+} \mathbf{g}^{\mathrm{t}} \frac{1}{\omega-\left(\boldsymbol{\lambda}^{\mathrm{t}}+\mathbf{Q}^{\mathrm{t}} \mathbf{V}^{\mathrm{t}}\left(\mathbf{Q}^{\mathrm{t}}\right)^{+} \mathbf{g}^{\mathrm{t}}\right)} \mathbf{Q}^{\mathrm{t}}, \\
& =\left(\mathbf{Q}^{\mathrm{t}}\right)^{+} \mathbf{g}^{\mathrm{t}} \mathbf{M}^{-1} \frac{1}{\omega-\boldsymbol{\eta}^{\mathrm{t}}(\mathbf{k})} \mathbf{M} \mathbf{Q}^{\mathrm{t}} .
\end{aligned}
$$

The step from the second line to the third can be shown by expanding the inverse [Aic $\left.{ }^{+} 06 \mathrm{~b}\right]$. In the last line the modal matrix $\boldsymbol{M}$ was introduced which diagonalizes $L^{t}=\left(\lambda^{t}+Q^{t} V^{t}\left(Q^{t}\right)^{+} g^{t}\right)$. The $\eta^{t}(k)$ denotes a matrix with the eigenvalues $\eta_{\alpha}^{t}(\mathbf{k})$ on the diagonal. These are wave vector dependent by virtue of the interaction matrix $V^{t}$.

Due to its derivation from equation (3.33) the term (3.39) can be viewed as a matrix containing approximations to the correlation functions of the 
original system. This view is supported by the general structure which was derived in the last line. If one sums over the wave vectors $k$ the eigenvalues $\eta_{\alpha}^{t}(k)$ represent an approximation to the excitations of the full system. This directly leads to a problem of the present approach. Since $\mathbf{Q}^{\mathrm{t}} \mathbf{V}^{\mathrm{t}}\left(\mathbf{Q}^{\mathrm{t}}\right)^{+}$is a hermitian matrix and $\mathbf{g}^{\mathrm{t}}$ has varying entries \pm 1 , the matrix $\mathrm{L}^{\mathrm{t}}$ is non-hermitian. This means that in principle eigenvalues that lie on the imaginary axis can be obtained, which is not in accordance with their supposed interpretation as approximated physical excitations. The possible imaginary poles will also pose mathematical problems for the evaluation of the SVCA free energy. This point will be discussed later in this chapter.

The next step is to carry out the traces in (3.35). It is clear that the Matsubara frequency sum will be the most challenging part. Usually, the correlation function (3.36) decreases relatively fast with $\omega_{l}$. Therefore, at large $T$ it is sufficient to consider only a finite number of terms. For $\mathrm{T} \rightarrow 0$ on the other hand the sum over the Matsubara frequencies can be carried out efficiently as a numerical integration [Sen08]. In the present approach however it is mandatory to devise an algorithm working at arbitrary temperatures, for reasons discussed in section 3.5. A suitable analytical technique was originally introduced for fermionic [Pot03a] and bosonic systems [KD06]. In the evaluation of the SVCA equations one can proceed along the lines of the latter because the correlation function of the spin operators has a structure similar to the bosonic Green function [FW71].

The evaluation starts with the term from (3.35) that only incorporates the matrix of cluster correlation functions and is thus independent of the wave vectors $\mathrm{k}$ :

$$
\operatorname{Tr} \ln \Pi_{\mathrm{c}}^{\mathrm{t}}=\frac{\mathrm{N}}{\mathrm{N}_{\mathrm{c}}} \sum_{i, \omega_{n}} \ln \pi_{i}^{\mathrm{t}}\left(\omega_{\mathrm{n}}\right),
$$

where the $\pi_{\mathfrak{i}}^{\mathrm{t}}\left(\omega_{n}\right)$ are the eigenvalues of $\Pi_{\mathrm{c}}^{\mathrm{t}}$. In evaluating these objects a subtlety arises: each contains a certain number of excitations in such a way that every individual $\lambda_{\alpha}^{t}$ defined in (3.37) appears only once. This is important to ensure a proper normalization of the trace.

Next, one has to perform the individual frequency sums over the terms $\ln \pi_{i}^{\mathrm{t}}\left(\omega_{n}\right)$. A standard way to do this is by means of Poisson's summation formula. An alternative approach suited for the terms appearing in (3.40) was introduced by Potthoff for fermions [Pot03a] and subsequently extended to bosons by Koller and Dupuis [KD06]. The latter scheme is applicable since spins follow commutator relations. The sum over the imaginary frequencies is performed using contour integration and analytical continuation to the real axis. A detailed derivation can be found in [KD06]. The result depends solely on the poles $\lambda_{\alpha}^{t}$ and zeros $\zeta_{\alpha}^{\mathrm{t}}$ of the functions $\pi_{\mathfrak{i}}^{\mathrm{t}}(\omega)$ :

$$
\operatorname{Tr} \ln \Pi_{c}^{\mathrm{t}}=\frac{\mathrm{N}}{\mathrm{N}_{\mathrm{c}}}\left(-\sum_{\alpha} \ln \left|1-e^{-\beta \lambda_{\alpha}^{\mathrm{t}}}\right|+\sum_{\alpha} \ln \left|1-e^{-\beta \zeta_{\alpha}^{\mathrm{t}}}\right|\right) .
$$


Note that the sum over the index $i$ has already been taken into account. For the derivation it is important that the correlation functions do not have a pole with non-vanishing weight at $\omega=0$. This is naturally the case for the transversal correlation function $\Pi^{\mathrm{t}}$, except for a $\mathrm{SU}(2)$ symmetric system.

Using a similar computation one finds for the $\mathrm{k}$-dependent part of (3.35):

$$
\begin{aligned}
& \operatorname{Tr} \ln \left(\left(\boldsymbol{\Pi}_{\mathrm{c}}^{\mathrm{t}}\right)^{-1}-\mathbf{V}^{\mathrm{t}}\right)^{-1}= \\
& \quad=\left(-\sum_{\alpha, \mathrm{k}} \ln \left|1-\mathrm{e}^{-\beta \eta_{\alpha}^{\mathrm{t}}(\mathrm{k})}\right|+\sum_{\alpha, \mathrm{k}} \ln \left|1-\mathrm{e}^{-\beta \nu_{\alpha}^{\mathrm{t}}(\mathbf{k})}\right|\right)
\end{aligned}
$$

The poles $\eta_{\alpha}^{t}(\mathbf{k})$ were found in (3.39) as the eigenvalues of the matrix $L^{t}$. The zeros $v_{\alpha}^{\mathrm{t}}(\mathbf{k})$ on the other hand are determined by the poles of the spin self energy $\Gamma^{t}$ defined in equation (3.21). In the central approximation of this approach that led to (3.33) one effectively uses $\Gamma_{\mathrm{c}}^{\mathrm{t}}$ as the self energy for both the cluster system as well as the approximated lattice system correlation function. So the collection of zeros $\left\{\zeta_{\alpha}^{t}\right\}$ is equal to $\left\{v_{\alpha}^{\mathrm{t}}(\mathbf{k})\right\}$ which means that the corresponding terms in (3.41) and (3.42) are equivalent. For (3.35) this leads to:

$$
\mathrm{K}^{\mathrm{t}}=\sum_{\alpha, \mathrm{k}} \ln \left|1-\mathrm{e}^{-\beta \eta_{\alpha}^{\mathrm{t}(\mathrm{k})} \mid}-\frac{\mathrm{N}}{\mathrm{N}_{\mathrm{c}}} \sum_{\alpha} \ln \right| 1-\mathrm{e}^{-\beta \lambda_{\alpha}^{\mathrm{t}}} \mid .
$$

To evaluate the longitudinal terms in (3.34) the corresponding cluster correlation functions are written in their Lehmann representation as:

$$
\begin{aligned}
\Pi_{i j}^{z}\left(\omega_{l}\right)= & \frac{1}{Z} \sum_{E_{n} \neq E_{m}} \frac{e^{-\beta E_{n}}-e^{-\beta E_{m}}}{\omega_{l}-\left(E_{n}-E_{m}\right)}\left\langle n\left|S_{i}^{z}\right| m\right\rangle\left\langle n\left|S_{j}^{z}\right| m\right\rangle \\
& +\delta_{l, 0} \frac{\beta}{Z} \sum_{E_{m}=E_{n}} e^{-\beta E_{n}}\left\langle n\left|S_{i}^{z}\right| m\right\rangle\left\langle n\left|S_{j}^{z}\right| m\right\rangle,
\end{aligned}
$$

where the self-adjoint character of $\mathbf{S}^{z}$ has been taken care of and the special contribution to the zeroth component been separated.

At this point one comment is in order. In the $K^{z}$-part of (3.34) the inverse and the logarithm of $\Pi^{z}$ appear. As is shown in appendix B this matrix has zero as an eigenvalue for all Matsubara frequencies except $\omega_{0}$ in case total $S^{z}$ is conserved. Thus, $\left(\Pi^{z}\right)^{-1}$ and $\ln \Pi^{z}$ individually have to be understood in principle as containing a small regularizing parameter $\epsilon$ to take care of these zero eigenvalues. However, it has been shown in appendix $B$ that the individual divergencies which develop for $\epsilon \rightarrow 0$ exactly cancel if one takes into account all terms in $\mathrm{K}^{z}$ respectively (3.34). Therefore the SVCA free energy as a whole is well-defined. Nevertheless, care has to be taken when the different contributions are evaluated individually. 
With this subtlety in mind the evaluation of the longitudinal parts is continued. The first line of (3.44) resembles terms that also appear in the transversal correlation function (3.36), and in principle one could perform a similar computation. Unfortunately the second line proves to be problematic. If one extends (3.44) to be a function on the complex plane it has a discontinuous point at the origin. This non-analyticity of $\Pi_{i j}^{z}(\omega)$ would render it impossible to evaluate the Matsubara frequency sum in the same way as in the transversal case. The contour integration which is performed to find (3.41) only leads to a proper result if the function is analytical at the origin [KD06].

So, one needs to introduce a matrix $\hat{\Pi}_{\mathrm{c}}^{z}$ which is defined by (3.44) but with the terms in the last line omitted. The corresponding functions are analytical at the origin. Since $\hat{\Pi}_{\mathfrak{c}}^{z}$ and $\Pi_{\mathfrak{c}}^{z}$ only differ for $\omega_{0}$ one can rewrite the expressions from (3.35) as:

$$
\operatorname{Tr} \ln \Pi_{\mathrm{c}}^{z}=\operatorname{Tr} \ln \hat{\Pi}_{\mathrm{c}}^{z}+\left.\operatorname{Tr} \ln \Pi_{\mathrm{c}}^{z}\right|_{\omega_{0}}-\left.\operatorname{Tr} \ln \hat{\Pi}_{\mathrm{c}}^{z}\right|_{\omega_{0}},
$$

and:

$$
\begin{aligned}
\operatorname{Tr} \ln \left(\left(\boldsymbol{\Pi}_{\mathrm{c}}^{z}\right)^{-1}-\mathbf{V}^{z}\right)^{-1}= & \operatorname{Tr} \ln \left(\left(\hat{\Pi}_{\mathrm{c}}^{z}\right)^{-1}-\mathbf{V}^{z}\right)^{-1} \\
& +\left.\operatorname{Tr} \ln \left(\left(\boldsymbol{\Pi}_{\mathrm{c}}^{z}\right)^{-1}-\mathbf{V}^{z}\right)^{-1}\right|_{\omega_{0}} \\
& -\left.\operatorname{Tr} \ln \left(\left(\hat{\boldsymbol{\Pi}}_{\mathrm{c}}^{z}\right)^{-1}-\mathbf{V}^{z}\right)^{-1}\right|_{\omega_{0}} .
\end{aligned}
$$

The first terms on the right hand side of (3.45) and (3.46) can now be evaluated as in the transversal case, with $\hat{\Pi}_{\mathrm{c}}^{z}$, the $\mathbf{Q}^{z}$-matrices and corresponding quantities like $\mathrm{L}^{z}$ defined in analogy to their transversal partners. The poles $\lambda_{\alpha}^{z}$ are obtained from the matrix (3.44) and the $\eta_{\alpha}^{z}(\mathrm{k})$ are determined by the eigenvalues of $\mathbf{L}^{z}$. Both of them are used in the formula corresponding to (3.43).

The last two terms from (3.45) and (3.46) which only give a contribution for $\omega_{0}$ can be combined and computed directly. Putting everything together one arrives at:

$$
\begin{aligned}
\mathrm{K}^{z}= & \sum_{\alpha, \mathrm{k}} \ln \left|1-\mathrm{e}^{-\beta \eta_{\alpha}^{z}(\mathrm{k})}\right|-\frac{\mathrm{N}}{\mathrm{N}_{\mathrm{c}}} \sum_{\alpha} \ln \left|1-e^{-\beta \lambda_{\alpha}^{z}}\right| \\
& +\sum_{\mathrm{k}} \ln \operatorname{det}\left(1-\mathbf{V}^{z} \Pi_{\mathrm{c}}^{z}\left(\omega_{0}\right)\right) \\
& -\sum_{\mathrm{k}} \ln \operatorname{det}\left(1-\mathrm{V}^{z} \hat{\Pi}_{\mathrm{c}}^{z}\left(\omega_{0}\right)\right) .
\end{aligned}
$$

For the last two terms the matrix identity $\operatorname{Tr} \ln M=\ln \operatorname{det} M$ was used. The matrices under the determinants cover the cluster site indices.

One has to add that in case of a SU(2)-symmetric system the treatment of the transversal part has to be performed of course identically to the longitudinal part. 
Expressions (3.43) and (3.47) enter in the SVCA free energy (3.34), which now can be computed explicitly. Besides the cluster system free energy $F_{c}$ and excitations $\lambda_{\alpha}^{\xi}$ one has to calculate the determinants in (3.47). One also needs to compute the eigenvalues of the $L^{\xi}$ which can become numerically challenging. These matrices have a rank of the order of excitations in the system and grow rapidly. Finally, the k summation appearing in the equations has to be performed and is done over a mesh of different sizes to reduce numerical errors.

\subsection{DISCUSSION OF THE SVCA METHOD}

In the previous sections a variational cluster approximation for Heisenberg models has been developed. Starting from a path-integral representation for a spin Hamiltonian a suitable Luttinger-Ward functional was constructed within a functional-integral framework. Here, generalized self-energies linked to the spin correlation functions were introduced as functionals. For the exact physical system they can in principle be identified as the Larkin self-energies, which are obtained as a result of the spin diagram formalism presented in chapter 2.2. This identification was also used by Ovchinnikov for developing a spin cluster perturbation theory [OBS10].

The spin Luttinger-Ward functional exhibits the important properties that are mandatory for further approximations, like certain stationary conditions. It was used to introduce the SVCA equations which are supposed to give approximations to the free energy of physical spin systems. Finally a method was presented to explicitly evaluate these equations depending on a certain cluster reference system and the corresponding variational parameters. The stationary points with respect to the parameters give an approximate free energy, from which other thermodynamical properties of the system can be derived.

So the SVCA developed in this way appears to be a reasonable scheme for approximating Heisenberg spin systems. Yet, it has to be tested if these approximations are indeed working, which is a topic of the next chapter. However, before this can be done certain points concerning the SVCA have to be discussed in detail.

It was already mentioned at the end of sections 3.1 and 3.3 that the local part of the Hamiltonian needs to be the same for the physical and the cluster reference system. This point is vital, since the definition of the free energy functional (3.18), its Legendre transform and the spin Luttinger-Ward functional (3.23) critically depend on this property. In particular, the SVCA equation (3.32) was set up by eliminating $\tilde{P}$, which is not possible otherwise. 
As introduced in section 3.1 the local part of the action (3.8) reads:

$$
\tilde{S}_{\text {loc }}\left[\mathrm{s}^{\eta}, \tilde{\mathbf{J}}^{z z}, \tilde{\mathbf{J}}^{-+}\right]=\mathrm{B}\left(\mathrm{s}_{i}^{\eta}\right)+\int_{0}^{\beta} \mathrm{d} \tau \sum_{i} h s_{i}^{z}(\tau) .
$$

Theses terms need to be unchanged which is naturally fulfilled for the Berry phase $B\left(s_{i}^{\eta}\right)$ since it is independent of the actual Hamiltonian. The only limitation here would be that the magnitude of the spins remains the same in the reference cluster system, which is natural and reasonable in any case. However, the second term of (3.48) imposes the restriction that the local magnetic fields $h$ are fixed during the variation. Also, no additional local fields can be included in the reference system. Only entries in the interactions $\mathrm{J}^{\xi}$ are eligible as variational parameters.

On the other hand, variational local fields have proven to be a valuable tool in conventional VCA approaches, necessary to study certain states and phases of a system [Dah ${ }^{+} 04 ; \mathrm{Aic}^{+} 04$; Sen08; KD06]. It is a limitation of the SVCA method that one cannot make such a use of local fields which are associated with single spin operators. At present the only possible solution is the introduction of local anisotropies like $\left(\mathbf{S}_{\mathfrak{i}}^{z}\right)^{2}$ in the cluster Hamiltonian. The accompanying variational parameters feature $\mathbf{J}_{\mathbf{c}}^{z}$ along the diagonal. However, introducing such terms does not lead to improved results for the systems which have been tested so far.

As stated in section 3.1, this limitation is also the reason why the connected longitudinal correlation function is not used in the action (3.8). In this case, the local part would be given by equation (3.13), where new terms proportional to $\left\langle\boldsymbol{S}_{i}^{z}\right\rangle$ are included. So this local action consisting of magnetic fields and Hartree-like terms has to remain unchanged for the physical and the reference system. The requirement can in general not be met for the open spin clusters treated so far. Hence, to develop a functional SVCA approach one has to use the action (3.8) with the longitudinal correlation function $\left\langle T_{\tau} S_{i}^{z}(\tau) S_{j}^{z}\left(\tau^{\prime}\right)\right\rangle$.

This leads to another point that needs to be discussed. Larkin's equations (3.20) for the correlation functions $\Pi^{z}$ and $\Pi^{\mathrm{t}}$ were originally introduced for a ferromagnetic model [VLP68a] and later extended to bipartite antiferromagnetic systems [PSS69]. Anisotropies can also be incorporated in this formalism since they only vary the strength of the interactions, not the structure of the diagrammatic expansions. Yet, the Larkin self-energy is defined as the collection of diagrams that are irreducible with respect to one interaction line. Naturally, just connected diagrams can be part of this collection. Therefore Larkin's equation only holds exactly for the connected longitudinal correlation function. This means that the self-energy $\Gamma^{z}$ is not to be taken from (3.21) if the action (3.8) is chosen to define the correlation function $\Pi^{z}$. Indeed for this choice it would rather be:

$$
\Gamma^{z}=\left(\Sigma^{z}\right)^{-1}\left(1-\Xi\left(\Pi^{z}\right)^{-1}\right),
$$

where $\Sigma^{z}$ is the Larkin self-energy and $(\Xi)_{i j}=<S_{i}^{z}><S_{j}^{z}>$. 
So one has two options within SVCA. Either to have a sound physical interpretation for the longitudinal self-energy. Of course, that would give a stronger foundation for the approach but would also include using a local part of the Hamiltonian which could not be kept fixed for the original and the reference system. In the end the SVCA equations would not be solvable. The other choice is to use a longitudinal spin self-energy that is not as well supported but leads to an approximation that can indeed be evaluated. The latter will be taken in this thesis. Yet, as will be seen in the next chapter, there are reasons to believe that this may contribute to worse results when the longitudinal part is included in the SVCA.

It is important to note that this dilemma is not relevant for the transversal correlation function. Here one can have a reasonable definition for the physical spin self-energy as well as solvable SVCA equations.

A second important issue with the SVCA is the potential breakdown of the method under certain circumstances. As pointed out in section 3.4, there exists the possibility to obtain complex poles in the approximate physical correlation functions. Since this should not be the case for a system in thermal equilibrium one can conclude that the SVCA method may not lead to meaningful results for specific reference cluster systems with certain sets of parameters. Although such a breakdown need not be related to physical effects in any way, a possible interpretation could be that it signals a phase transition. The finite imaginary parts of the poles do not appear suddenly, but usually evolve continuously from the real axis to the imaginary axis by crossing the origin. However, as discussed in section 3.4, one has to demand that for consistency reasons there is no pole at zero frequency [KD06]. In bosonic systems a non-vanishing excitation at the complex frequency origin signals the development of a condensed phase.

For example, in VCA calculations for the Bose-Hubbard model, where breakdowns are also encountered, they are identified as a signal for the appearance of a superfluid phase [KD06]. This can be amended by formulating the VCA using a 'pseudo-particle' approach [KAL11] or more rigorously within the Nambu formalism [AKL11]. Both methods give valuable insight how condensed phases of bosonic systems can be treated in a variational cluster approximation. Although the physics of the spin operators is entirely different, it is tempting to invoke a similar interpretation for the breakdown of the approximation behind the SVCA, meaning that a different magnetic phase evolves for which a certain cluster structure is not suited any more. If this is true, one should in principle be able to set up a generalized cluster Hamiltonian that respects such a phase transition.

Such a breakdown is not a mere feature of the Q-matrix formalism or the specific computation presented in the last chapter. Generally, in this case the terms $\operatorname{Tr} \ln \left(\mathbf{1}-\mathbf{V}^{\xi} \boldsymbol{\Pi}_{\mathfrak{c}}^{\xi}\right)=\ln \operatorname{det}\left(\mathbf{1}-\mathbf{V}^{\xi} \boldsymbol{\Pi}_{\mathfrak{c}}^{\xi}\right)$ from (3.35) yield non-physical results due to negative determinants, regardless of the spe- 
cific way to evaluate the free energy.

One further comment has to be added. The breakdown we just discussed takes place below certain temperatures, which means that often $\mathrm{T}=0$ cannot be reached. This is the reason why it is advisable to evaluate the free energy (3.33) using a method suitable for finite temperatures as described in section 3.4.

When discussing the results of the SVCA for different models in chapter 4 the breakdown of the method will be treated in detail. It is valuable to examine for which cluster system and parameter space the complex poles appear for a given Hamiltonian. The interpretation of these results can give insight into the physical conditions of the original model. 


\section{RESULTS OF THE SPIN VCA}

In the last chapter the steps for the development of the SVCA were laid out in detail. Finally, a set of SVCA free energy equations were derived which need to be evaluated for spin systems of interest. To be more precise, certain sets of cluster reference systems have to be chosen and one has to search for stationary points with regards to cluster variational parameters. This yields an approximate value for the free energy, which can be used to derive other thermodynamical quantities of the original model. The procedure will be done in this chapter for two Heisenberg systems.

To test our method we first use the antiferromagnetic spin- $1 / 2$ chain with nearest neighbour interactions and an applied magnetic field. The model is well understood, so we can compare our results with the exact ones to estimate the value of our approximation. This is laid out in sections 4.1 and 4.2, which have been partially published in [FP14]. However, the content has been revised and expanded. We will also discuss the implementations of the approximation with regards to variational parameters and cluster size. The chapter is concluded by a section which discusses the SVCA results for the frustrated antiferromagnetic zig-zag ladder model. 
As mentioned above the test model we use is the antiferromagnetic spin-1/2 Heisenberg chain with nearest neighbour interaction. It has been studied extensively and can be treated exactly via the Bethe-ansatz [Bet31; Bax82]. The spin chain including an applied magnetic field was solved by analytical and numerical methods [Klu98; Tak09]. It will therefore be a good model to test the approximation since we can compare our results with the exact solutions by Klümper [Klu98] and thereby get valuable insight to the workings and worth of the SVCA. This section explains how the approximation is applied for the spin chain as well as discusses the advantages and disadvantages of specific SVCA implementations and reference systems. The results are presented in section 4.2.

We use the Hamiltonian of the isotropic Heisenberg chain with $\mathrm{N}$ sites and periodic boundary conditions

$$
\mathbf{H}=\sum_{i} h \boldsymbol{S}_{i}^{z}+\sum_{i} J\left(S_{i}^{z} S_{i+1}^{z}+\frac{1}{2}\left(S_{i}^{+} S_{i+1}^{-}+S_{i}^{-} S_{i+1}^{+}\right)\right) .
$$

To apply our approximation, we tile the chain into clusters of a certain size. One can also add auxiliary sites to the clusters as discussed in section 3.3, thus enlarging the Hilbert space. These would allow for additional variational parameters which could in principle enhance the approximation by simulating a connection to a bath. Yet it is a priori not clear that this will actually be the case. Several examples of clusters are shown in fig. 4.1. The Heisenberg chain sites are denoted as black circles while the auxiliary sites are hollow. Except for example (a) we introduce spatially uniform cluster interactions $J_{\mathcal{c}}^{z}$ and $J_{c}^{t}$, which act as variational parameters. The auxiliary interactions are given by $\mathrm{J}_{A}$.

Initially the cluster type (c) from fig. 4.1 was tested without adding additional sites. By comparing with (a) and (b) we could not observe any significant improvement in the results. So for the further treatment of the spin chain, we will not use auxiliary sites. Examples like (d) from fig. 4.1 which have an odd number of sites lead to a dangling spin which prohibits singlet formation in the individual cluster. This induces an unreliable approximation of the antiferromagnetic spin chain at least for small $h$ and $T$, which is to be expected. The physics of the original lattice cannot be captured properly by such a reference system. So after ruling out auxiliary sites and odd numbers of spins we will in the following use clusters of two (type (c)), four (type (e)) and six sites to examine the antiferromagnetic chain. The interaction matrices $\mathbf{V}^{\xi}$ used in the computations, which depend on these cluster choices, are given in appendix C. 


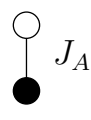

(a)

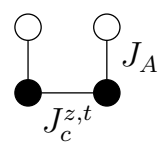

(b)

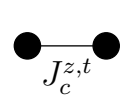

(c)

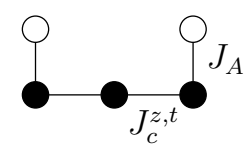

(d)

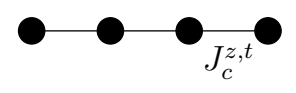

(e)

Figure 4.1: Several examples of clusters that could be used for the reference system of a spin chain. Additional sites are shown with hollow circles interacting via $J_{A}$. The parameters $J_{\mathcal{C}}^{z, t}$ denote the longitudinal and transversal interactions between the cluster lattice sites.

For each of these cluster reference systems, we need to evaluate the SVCA free energy (3.33) as described in section 3.4. With the notation introduced there, it reads as:

$$
\beta \mathrm{F}_{\mathrm{SVCA}}\left(\mathrm{J}_{\mathrm{c}}\right)=\beta \mathrm{F}_{\mathrm{c}}+\mathrm{K}^{z}+\mathrm{K}^{\mathrm{t}},
$$

with $\mathrm{K}^{\mathrm{t}}=\operatorname{Tr} \ln \left(\frac{\Gamma_{\mathrm{c}}^{\mathrm{t}}-\mathrm{J}^{\mathrm{t}}}{\Gamma_{\mathrm{c}}^{\mathrm{t}}-\mathbf{J}_{\mathrm{c}}^{\mathrm{t}}}\right)$ and $\mathrm{K}^{z}=\operatorname{Tr} \ln \left(\frac{\Gamma_{\mathrm{c}}^{z}-\mathbf{J}^{z}}{\Gamma_{\mathrm{c}}^{z}-\mathbf{J}_{\mathrm{c}}^{z}}\right)$. Here, $\mathbf{J}_{\mathrm{c}}^{\mathrm{t}}$ and $\mathbf{J}_{\mathrm{c}}^{z}$ are matrices constituted by the cluster interactions introduced via the reference system. Explicit expressions for the two quantities $\mathrm{K}$ are given in section 3.4, equations (3.43) and (3.47). As was laid out in that section, one needs the eigenvectors and -values of the cluster Hamiltonian to evaluate (4.2). We find these in the following by using full diagonalization.

As an example, we show in fig. 4.2 the results of an evaluation of the SVCA free energy for a two-site cluster and a magnetic field $h / J=1$. The cluster interaction is chosen to be isotropic, so the only variational parameter is $\mathrm{J}_{\mathrm{c}}^{z}=\mathrm{J}_{\mathrm{c}}^{\mathrm{t}} \equiv \mathrm{J}_{\mathrm{c}}$. To obtain a better picture of the relevant features, the difference $F_{S V C A}-F_{c}=T\left(K^{t}+K^{z}\right)$ is plotted.

Some characteristic properties can be discussed with the help of this plot. In VCA approaches, one generally searches for stationary points of thermodynamical functionals or functions with respect to the variational quantities. It can be easily seen in fig. 4.2 that extremal points exist for some temperatures, at least down to $\mathrm{T}=0.4 \mathrm{~J}$. With decreasing temperature, we observe that the algorithm does not give meaningful results for certain regimes of $\mathrm{J}_{\mathrm{c}}$ in accordance with the arguments in sections 3.4 and 3.5. In such cases no SVCA free energy is plotted in fig. 4.2 since the results are meaningless. Note that the regions where the algorithm breaks down become more and more extended as the temperature decreases, until we finally do not find any reasonable solutions to the SVCA any more. When this is happening for a certain T, the approximation as a whole fails. In the present example, this takes place around $\mathrm{T}=0.4 \mathrm{~J}$. For those regions where the algorithm works, we obtain smooth curves with well developed extremal points.

The fact that these are actually maxima and not minima of the free energy might seem odd at first. Yet this is often encountered in VCA approaches [Pot12a] and is a direct consequence of the derivation of equation (3.33). We are not searching for a minima of the free energy, but a 


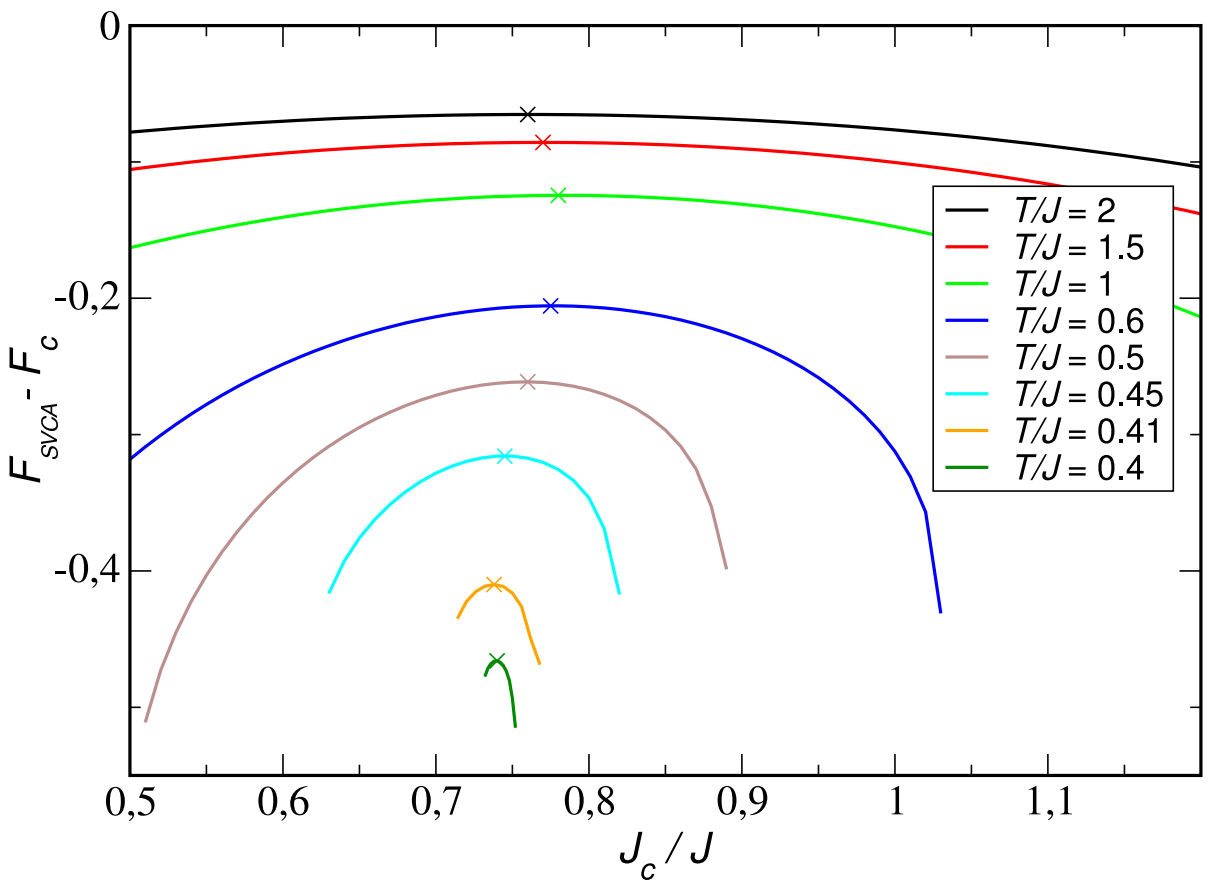

Figure 4.2: The difference $F_{S V C A}-F_{c}$ between the SVCA and the cluster system free energy per spin according to (4.2) for an antiferromagnetic spin chain using two-site clusters. It is plotted as a function of the intra-cluster exchange interaction $\mathrm{J}_{\mathrm{c}} / \mathrm{J}$ for $\mathrm{h} / \mathrm{J}=1$ and several temperatures. The maxima of the curves are indicated by crosses.

general stationary point.

We note that in the results shown in fig. 4.2, we did not observe a significant dependence on the density of points in the wave vector $k$ summation which we performed in the evaluation (see section 3.4). Yet, we encounter such a problem when we reach the parameter regime where the algorithm does not converge any more.

Once the free energy $F_{S V C A}\left(J_{c}\right)$ has been calculated, we need to determine the location $\hat{J}_{c}$ of the maxima. Within the present implementation of the algorithm this can be done with an accuracy of order of $10^{-3}$. This also provides an estimate of the numerical error of the computation since the errors of the full diagonalization routines are some orders of magnitude smaller. Within the SVCA the extremal point $F_{S V C A}\left(\hat{J}_{c}\right)$ provides an approximation to the physical free energy of the system. We can use it to derive other thermodynamical quantities, for example the magnetization as the derivative $\frac{\partial F}{\partial h}$, which is calculated numerically via the central difference. By scanning the parameter space we can then determine these thermodynamical quantities as functions of the temperature $T$ and the magnetic field.

In fig. 4.3 the magnetization per spin for a magnetic field $h=3 \mathrm{~J}>$ $h_{\text {sat }}=2 \mathrm{~J}$ obtained from a two-site cluster SVCA is plotted versus temperature. The exact solution derived with the Bethe ansatz shows that 


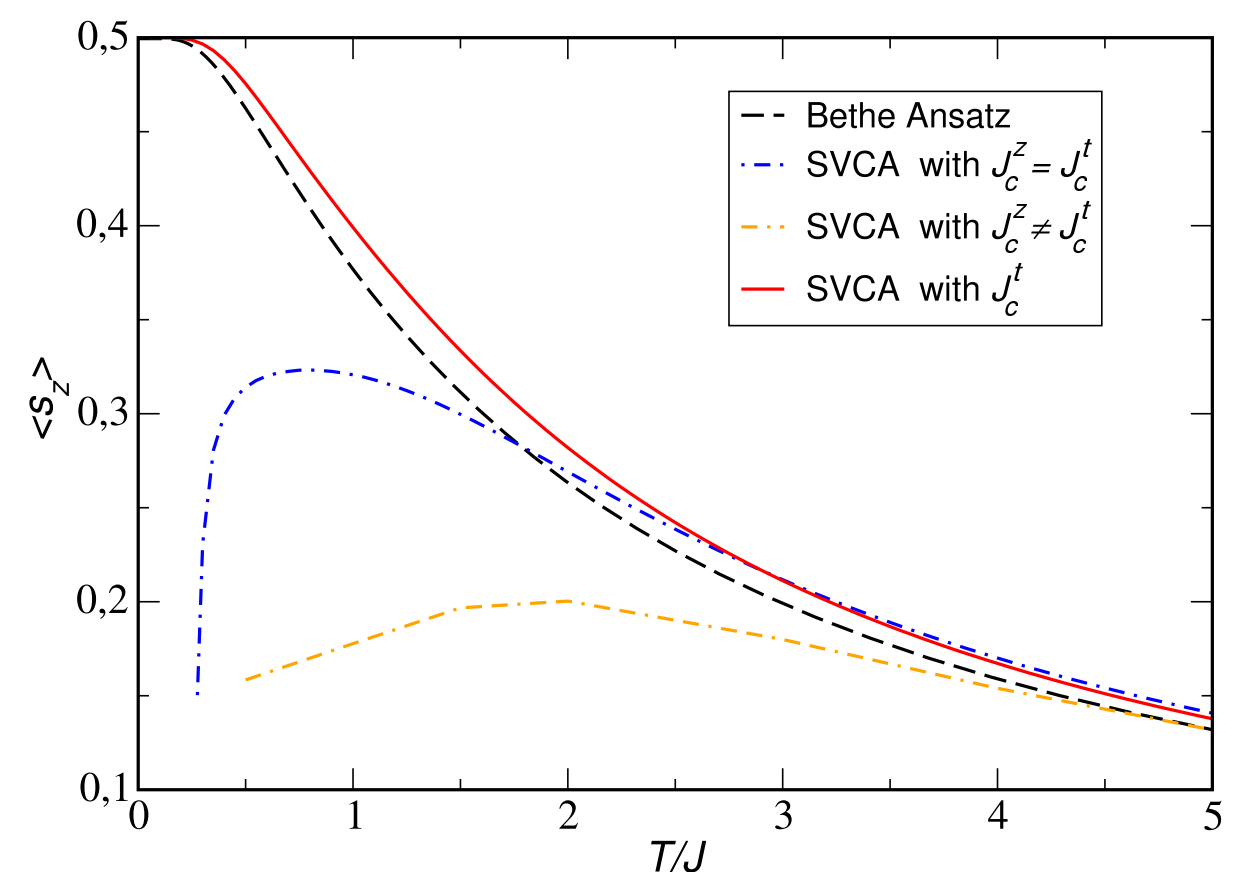

Figure 4.3: The magnetization per spin as function of $T / \mathrm{J}$ for the antiferromagnetic spin chain with magnetic field $h=3 \mathrm{~J}$. The exact Bethe Ansatz solution [Klu98] is compared with SVCA results for a two-site cluster system and different choices of variational parameters. The low-temperature behaviour of the second and third curve is omitted for convenience.

the magnetization saturates for small $\mathrm{T}$ [Klu98]. The region has been chosen since we do not expect a breakdown of the SVCA approximation for this large magnetic field. Fig. 4.3 compares the exact solution with our results for several choices of variational parameters. We tested the isotropic case $J_{c}^{t}=J_{c}^{z}$ which was already used above as well as the case where $J_{c}^{t} \neq J_{c}^{z}$ are two independent parameters. The latter means that we have to find a stationary point in a two-dimensional parameter space, which naturally is numerically more challenging. For the third choice presented in fig. 4.3 we set $J_{\mathfrak{c}}^{z}$ equal to the original Heisenberg lattice $J$. This means we only vary the transversal cluster interaction $J_{\mathrm{c}}^{\mathrm{t}}$.

Obviously, this selection of variational parameter yields the best result when compared to the exact solution. The isotropic and even more so the two-dimensional anisotropic variation lead to clearly wrong magnetization curves, showing a suppression of $\left\langle\mathrm{S}_{z}\right\rangle$ for lower temperatures. The technical reason for this behaviour is that when we use $J_{c}^{z}$ and $J_{c}^{t}$ as independent variational parameters, the extremal points of $F_{S V C A}$ appear at values up to $\mathrm{J}_{\mathrm{c}}^{z} \approx 3.5 \mathrm{~J}$ for low temperatures, while $\mathrm{J}_{\mathrm{c}}^{\mathrm{t}}$ stays between $0.6 \mathrm{~J}$ and $0.8 \mathrm{~J}$. As a consequence the approximation is dominated by artificially enhanced antiferromagnetic correlations counteracting the external magnetic field, which can be clearly seen in fig. 4.3. In the isotropic case, i.e. if we vary the two parameters together, the transversal part holds the longitudinal part at bay. Here, values range between $\mathrm{J}_{\mathrm{c}} \approx 0.8 \mathrm{~J}$ 
for $\mathrm{T}=5 \mathrm{~J}$ and $\mathrm{J}_{\mathrm{c}} \approx 1.5 \mathrm{~J}$ for low $\mathrm{T}$. Yet the associated magnetization curve for lower temperatures is still worse than in the last case. If we only vary $J_{c}^{t}$ the extremal points of $F_{S V C A}$ are found at values that always lie below $0.9 \mathrm{~J}$, which obviously leads to a better approximation of the spin chain thermodynamics.

It may at first seem odd that restricting the variational degrees of freedom results in a better representation of the physics. Yet, there are actually several reasons why this observation makes sense.

Firstly, for one-dimensional spin models the spin operators can be mapped onto fermionic operators by the Jordan-Wigner transformation [JW28], which was laid out in section 2.3. As can be seen from equations (2.33)-(2.39), the transversal terms $\mathbf{S}^{+} \mathbf{S}^{-}$under this mapping become the kinetic energy of the new Hamiltonian while the longitudinal terms $\mathbf{S}^{z} \mathbf{S}^{z}$ lead to density-density interactions of the fermions. However, for the fermionic version of the VCA, we know that variational parameters connected to the kinetic energy lead to sensible approximations, while the local interaction part is to be kept fixed (see section 2.1). Although we face the additional complexity of non-local interactions, one can take this comparison as a hint that the longitudinal parameters are not well suited for variation.

Secondly, we believe the behaviour of $\mathrm{J}_{\mathcal{c}}^{z}$ is related to the magnetic field $h$ applied in the $z$-direction. As discussed in chapters 3.4 and 3.5 we cannot use $h$ as a variational parameter, i.e. it has to remain fixed. In the fermionic or bosonic version of the VCA local fields can be used to enforce thermodynamical consistency between cluster and real system, for example with respect to the occupation number in electronic systems [Aic $\left.{ }^{+} 06 \mathrm{a} ; \mathrm{Sen} 08\right]$. As in the present formulation of the SVCA we do not have such direct control over the magnetization in $z$-direction through a variation of local magnetic fields, the SVCA seems to maximize the effect of the fixed external field by strongly increasing the longitudinal interaction $\mathrm{J}_{\mathrm{c}}^{z}$. This behaviour is encountered generally in our SVCA computations.

One should also take another aspect of this latter point into account, which is more concerned with the development of the approximation. The fact that we needed to keep $h$ fixed in the variation leads to problems defining the longitudinal self-energy in a fully sound physical way (see section 3.5). In contrast to the transversal interaction it had to be a formally defined quantity. The self-energies play a vital role in the derivation of the SVCA equations and approximations are in principle implemented directly on this level. It is a priori not clear why these should lead to good results if the self-energy itself is not accessible on a physical, respectively perturbative, level. Thus, it should not surprise that approximations using longitudinal parameters are less reliable.

Given the test results plotted in fig. 4.3 and the subsequent interpretation and discussion it is sensible to keep $J_{c}^{z}=J$ fixed, using only $J_{c}^{t}$ as the variational parameter. All results presented in the next sections were obtained in this way. However, this does not mean that $\mathrm{K}^{z}$ from 
(4.2) can be neglected in the computation. It still has to take account of the fact that there is no longitudinal inter-cluster interaction in the reference system. The matrix $\mathbf{V}^{z}=\mathbf{J}^{z}-\mathbf{J}_{\mathrm{c}}^{z}$ in (3.34) has non-zero entries. So there is a small but finite contribution of $K^{z}$ to the SVCA free energy even when $J_{c}^{z}$ is kept fixed.

\subsection{ANTIFERROMAGNETIC SPIN CHAIN}

The last section discussed specific variants of implementing the SVCA in order to find the most sensible and viable choice of parameters. Consequently, the results obtained for the antiferromagnetic Heisenberg chain were calculated using reference systems with the transversal interaction $J_{c}^{t}$ as the variational parameter and clusters of two, four and six sites. The latter is done to compare the influence of different cluster sizes.

Figure 4.4 shows the magnetization curves as a function of temperature for four different magnetic fields, including the critical $h_{\text {sat }}=2 \mathrm{~J}$. Each plot in fig. 4.4 contains the exact Bethe ansatz solution and SVCA results for the three different cluster sizes. For larger fields, the magnetization per spin is known to smoothly saturate to $1 / 2$ [Gri64]. This region is represented in fig. 4.4 by a plot for $h=3$ J. When $h<2$ J, the magnetization curves go through a maximum to settle for a finite value less than $1 / 2$ as $T \rightarrow 0$. Two graphs show results for magnetic fields below the critical field.

As can be seen in fig. 4.4, the SVCA approximation yields the most precise results for the largest magnetic field, $h=3 \mathrm{~J}$. This holds true in general for any $h>2 \mathrm{~J}$, where the magnetization saturates. In this case we also find that the approximation remains stable down to $\mathrm{T}=0$. The saturation value of $1 / 2$ is found numerically within good precision. This supports our choice to only vary $J_{c}^{t}$. The dependence on cluster size appears to be very mild - the curves for the four- and six-spin systems already nearly coincide. Thus, at least in the case of larger magnetic fields reliable approximations can be obtained for small to moderate cluster sizes.

The results for the critical value $h=2 J$ seem to behave similarly at first glance, in particular the approach to the value $1 / 2$ for $T=0$. However, the specific form of the exact solution with its non-analyticity at $\mathrm{T} \rightarrow 0$ is not captured properly. Moreover, from the kink in the magnetization curve at low $\mathrm{T}$ for the two-site cluster one can infer the appearance of additional irregular behaviour. This is a general feature in all our SVCA calculations close to the critical field, which is more or less pronounced depending on the actual quantity under consideration. For example, it is extremely prominent in the specific heat in fig. 4.5. Finally, for $\mathrm{h}<2$ J our approximation starts to break down at some finite temperature, as can be seen in fig. 4.4. It thus seems that the SVCA indeed realizes that a special situation arises for the model at $h_{c}$. 

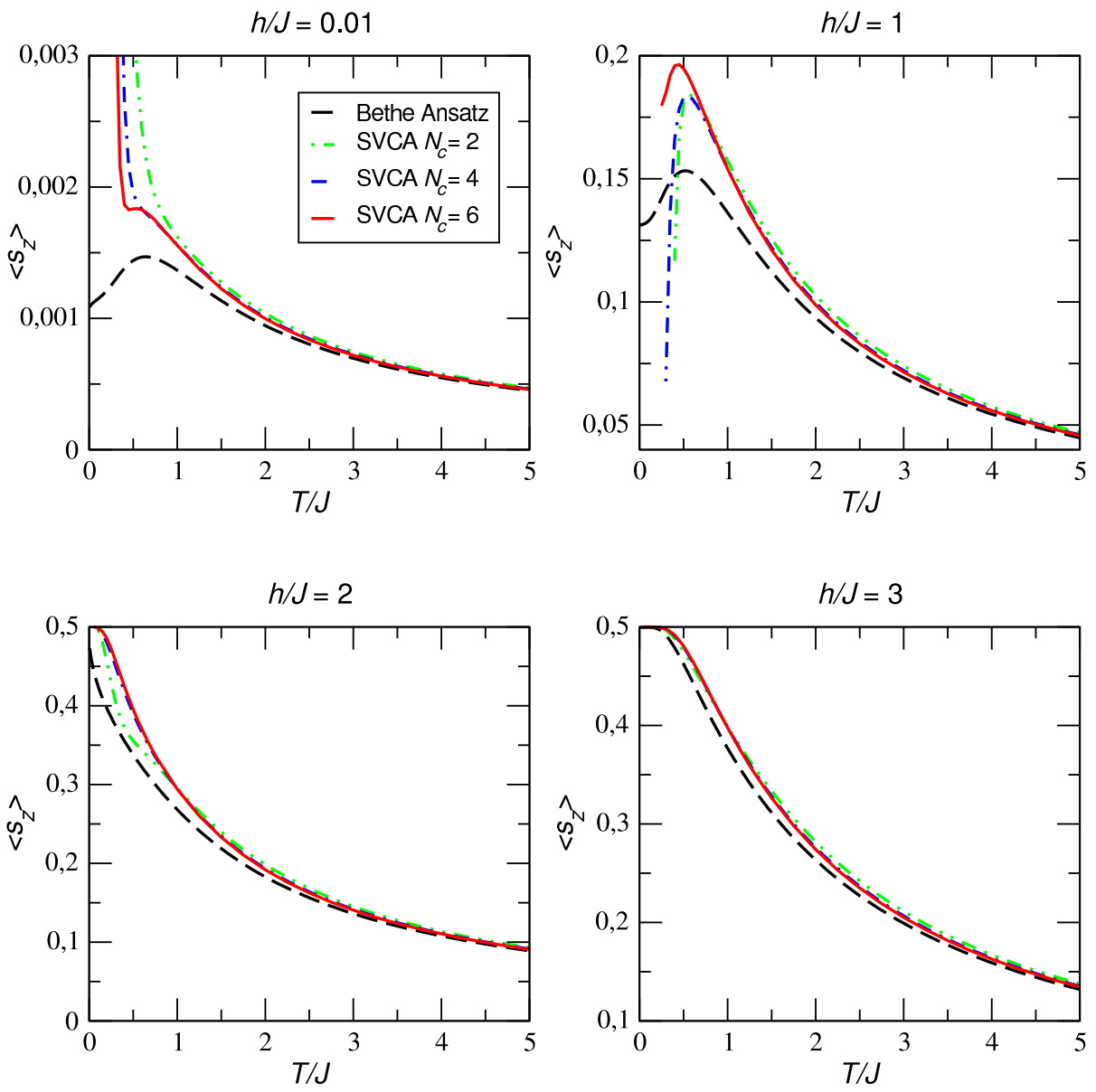

Figure 4.4: The magnetization per spin as a function of the temperature derived with the SVCA for four different magnetic fields. In each graph the results for three cluster choices are plotted together with the exact Bethe ansatz solution. 
At $h / J=0.01$ and $h / J=1$, zero temperature can not be reached any more as the SVCA starts to develop complex poles in the propagators for the whole parameter space and the results become meaningless. The mechanism of this breakdown has been introduced in 3.4. Details of the process were also discussed in the context of fig. 4.2 in 4.1. However, we note that with increasing cluster size the stability region also increases and solutions are found for lower T. Indeed, especially the curves for the four- and six-spin cluster do not deviate much from one another, until at a certain point, the solution for the smaller system breaks down. The larger one continues to provide reasonable results down to lower $\mathrm{T}$, until it also starts to become unstable. This behaviour shows that the SVCA indeed is an approximation that at least improves systematically with cluster size. Note that this can in principle be expected from the formulation of the method, but is nevertheless by no means trivial.

As can be seen in the plots, the magnetization usually becomes divergent to either plus or minus infinity when the SVCA breaks down. It is tempting to interpret this breakdown as a signal for a phase transition, albeit an artificial one.

The antiferromagnetic Heisenberg spin chain does not have a true phase transition at any temperatures [MW66], but is critical in the sense that it develops algebraic correlations at $\mathrm{T}=0$ [Faz99]. Adding a not too large magnetic field does not change this situation, but only results in a finite magnetization less than 1/2 [Faz99; Klu98].

Since cluster theories such as the SVCA tend to produce phase transitions if the correlations in the clusters become too strong, we suggest that the approximation here tries to form an ordered state to accommodate the slow decay of correlations in the cluster. As the analytical structure of the quantities entering the SVCA should be different in such a situation, we can not expect that our present implementation is suitable to handle it properly. In particular the appearance of long-range magnetic order will lead to consistency problems as discussed in section 3.5.

To conclude, the SVCA for small clusters is apparently not able to properly describe the region where the spins form some correlated, nonsaturated state at low temperature. However, the curves in fig. 4.4 for the six-spin cluster at least show the maximum of the magnetization present in the exact solution, although the magnetization values for both $h=0.01 \mathrm{~J}$ and $\mathrm{h}=\mathrm{J}$ are systematically too high, overestimating the exact value in the maximum by $\approx 25 \%$. Yet it is interesting that the positions of the maxima are close to their exact values: For $h=0.01 \mathrm{~J}$ the SVCA predicts $\mathrm{T}_{\text {max }, \mathrm{SVCA}} \approx 0.55 \mathrm{~J}$ to be compared with $\mathrm{T}_{\max , \mathrm{BA}} \approx 0.65 \mathrm{~J}$, while for $h=J$ we obtain $T_{\max , \mathrm{SVCA}} \approx 0.44 \mathrm{~J}$ versus $\mathrm{T}_{\max , \mathrm{BA}} \approx 0.5$. Thus the SVCA with our present set-up seems to systematically overestimate the magnetization for lower temperatures - this is also true for larger magnetic fields, albeit not that strongly. On the other hand, it underestimates the fluctuation scale related to the position of the maximum. Note that both features are related and the directions they are going consistent. As mentioned earlier, such a behaviour could in principle be expected 
generically because we do not have the option to adjust a local field as control parameter to enforce a certain magnetization value. For temperatures smaller than $T \approx 2$ J, the SVCA results seem to be more appropriate for a higher magnetic field than the one from the actual lattice Hamiltonian. Indeed, the position of the maximum of the magnetization for $\mathrm{h}=\mathrm{J}$ discussed above approximately fits the Bethe Ansatz result for $h=1.25 \mathrm{~J}$ [Klu98]. This overestimation by up to $25 \%$ is a consistent behaviour for low temperatures in our results. It indicates that in this regime the SVCA free energy behaves as if the magnetic field were larger than it actually is.

This might be amended if magnetic fields could be variational parameters to control the thermodynamical consistency of the magnetization. Since they are not available within the present formalism one can only assume this for the moment.

In a different point of view, the specific low temperature behaviour of the SVCA results could also be related to finite size effects, which naturally appear in exact diagonalization computations for spin clusters [BF64]. They show certain irregular behaviour in the very low temperature regime. Since the SVCA is derived as a dynamical embedded cluster approach one would hope that finite size effects are less pronounced. Although they might play a role, the consistent 'spiking' behaviour of the magnetization for low temperatures in the sub-critical region points in a different direction. This is also supported by the fact that the parameter $\mathrm{J}^{\mathrm{t}}$ at the stationary point often changes rapidly for $\mathrm{T}<1.5 \mathrm{~J}$. So the results for low temperatures in the sub-critical regime are not dominated by classical finite size effects. Rather, the feature seems to be inherent to the approximation and needs to be further investigated.

To conclude the discussion of the SVCA results for the antiferromagnetic spin chain, we show in fig. 4.5 our results for the specific heat as a further example. The cluster sizes and parameters used are the same as for the magnetization in fig. 4.4. To find the heat capacity $c=-T \frac{\partial^{2} F}{\partial T^{2}}$, one needs to numerically calculate the second derivative, which is more prone to errors. From the plots in fig. 4.5, we directly see that the SVCA results for the heat capacity are less accurate than for the magnetization, even for larger magnetic fields. For the critical value $h=2 J$, we find additional artificial structures at low temperatures. Increasing the cluster size improves the agreement with the exact Bethe ansatz curve down to roughly $\mathrm{T} \approx 1.4 \mathrm{~J}$, but deviations remain significant for lower $\mathrm{T}$ and do not seem to improve systematically. The situation becomes even worse for $h<2 J$, where again divergencies appear and also the overall shape is not reproduced well any more. At least for $h=J$ the tendency follows the expectation, viz that increasing the cluster size yields a systematic improvement of the results - but for $h=0.01 \mathrm{~J}$ even this feature seems to be lost. While one can expect that deficiencies of the approximation as well as numerical errors are more pronounced in quantities which are obtained as higher derivatives, it is not clear at the moment why the 

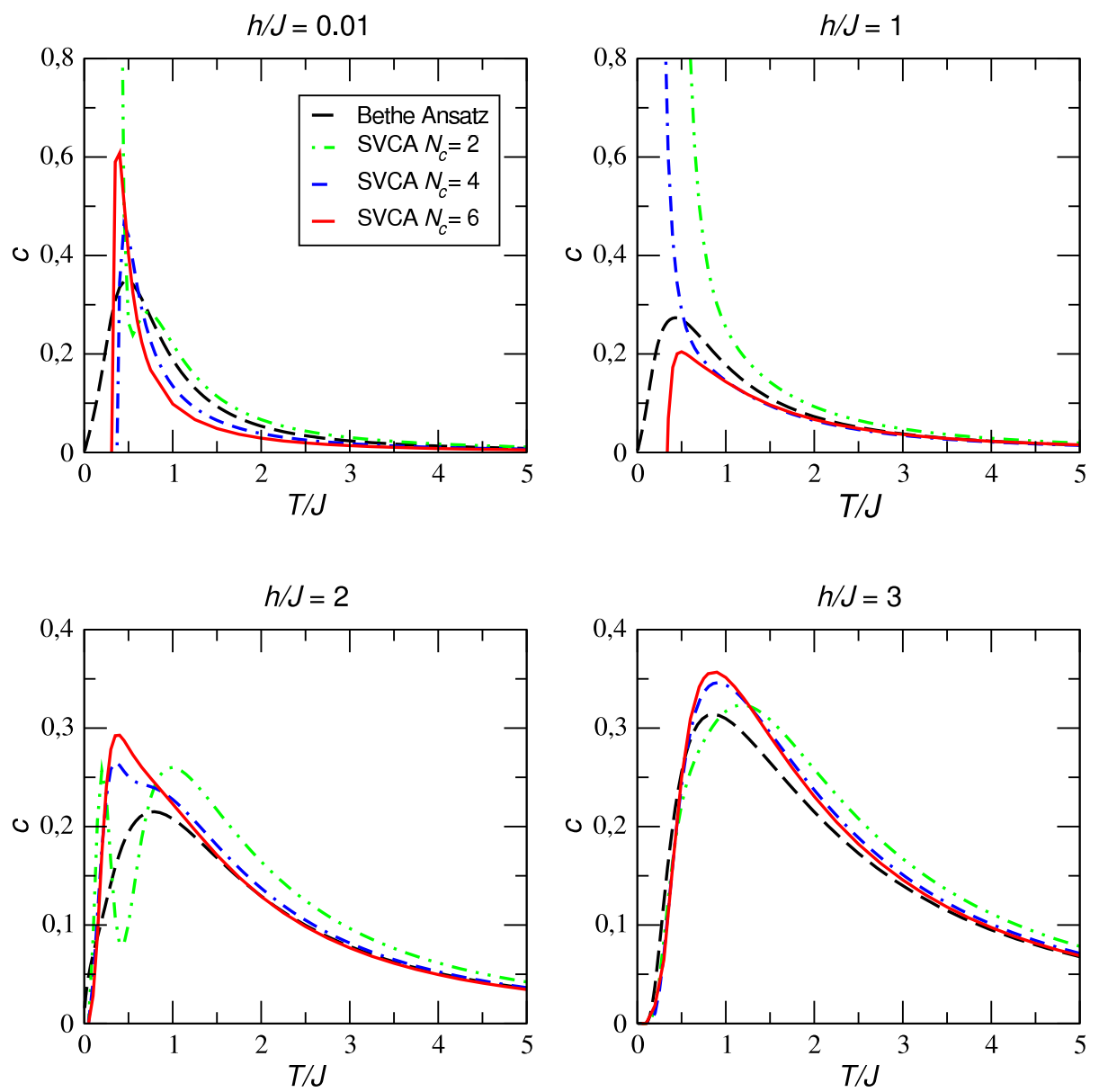

Figure 4.5: The heat capacity per spin is shown as a function of the temperature derived with the SVCA for four different magnetic fields. In each graph the results for three cluster choices are plotted together with the exact Bethe ansatz solution. 


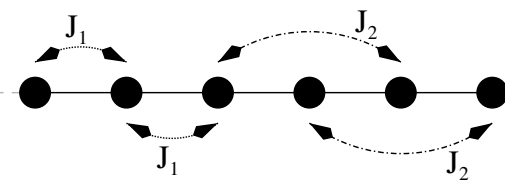

(a)

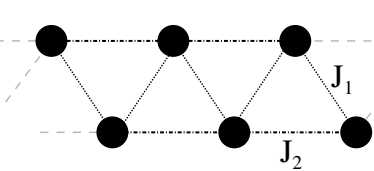

(b)

Figure 4.6: The figure shows a spin chain with additional next-nearest neighbour interactions (a). The system can be realized as a zigzag ladder (b). Due to its two interactions it is called $\mathrm{J}_{1}-\mathrm{J}_{2}$ model.

scaling with cluster size of the heat capacity significantly deviates from the expected behaviour for small magnetic fields.

In the case of a large magnetic field $h=3 \mathrm{~J}$, we again can calculate the specific heat down to $T=0$ and the curves approximate the exact solution reasonably well. As expected, the largest cluster provides the best approximation. Again, the SVCA results tend to overestimates the heat capacity around the maximum, but predict its position with good accuracy, which is improving with increasing cluster size. This confirms our previous observation from the magnetization that for magnetic fields above the critical field the SVCA results are more reliable than below $h=2 J$.

\subsection{RESULTS FOR A FRUSTRATED MODEL}

The results for the antiferromagnetic spin chain are interesting and give a first benchmark for the SVCA. It promises to be a reasonable approximation as long as the long-ranged algebraic correlation are not too strong. Since these become predominant in the chain for decreasing fields and temperatures, it should not be surprising that here we face problematic SVCA results. So it is apparent that one should test the approximation for a model where the long-ranged correlations are less prevalent than in the antiferromagnetic spin chain. This is of course the case in frustrated systems with often dominating short-ranged correlations for low temperatures. Two-dimensional antiferromagnetic examples can be found in the paper by Richter et. al. [RSH04].

One specific one-dimensional example is the $\mathrm{J}_{1}-\mathrm{J}_{2}$ spin chain with next nearest neighbour interactions. It can also be seen as a Heisenberg zigzag ladder. In case of antiferromagnetic coupling, the system is frustrated. A special choice for the couplings is the Majumdar-Ghosh model where the first-neighbour interaction is half as strong as the secondneighbour interaction and the ground state are dimers on a rung [MG69; MK04]. The model is depicted in two realizations in fig. 4.6.

There are two reasons why the antiferromagnetic spin chain with nextnearest neighbour interactions seems to be a good model for further ex- 
amination of the SVCA. First, as mentioned before, it is dominated by short-ranged correlations in the low-temperature regime. Given the discussion at the beginning of the section, this is a prominent candidate model for the next step in the testing of the approximation. Second, the $\mathrm{J}_{1}-\mathrm{J}_{2}$ model is an extension of the normal spin chain and can be easily treated with a similar analytical and numerical set-up. This is an advantage over two-dimensional frustrated lattices, where the numerical effort becomes much bigger. Generally, it is interesting to look at ladder systems in cluster-based approximations as the next step beyond one-dimensional models. On the one hand, connections to two dimensional systems can be found like in the case of the $\mathrm{J}_{1}-\mathrm{J}_{2}$ chain and the Shastry-Sutherland model, where dimer-singlet ground states are found [SS81; $\left.\mathrm{Hon}^{+} 16\right]$. On the other hand, they are treatable as systems with a one-dimensional meta-lattice along which the clusters are aligned. Regarding the SVCA this means that we keep the computational effort of evaluating the free energy limited by only summing over a scalar $k$.

The fully frustrated spin ladder - treated for example in the paper by Honecker et al. [Hon ${ }^{+} 16$ ] - would be more interesting, yet we will restrict ourselves to the $\mathrm{J}_{1}-\mathrm{J}_{2}$ chain for the purpose of testing the SVCA. This model, depicted in fig. 4.6 (b), can be seen as the most simple ladder system. Its Hamiltonian can be written as:

$$
\begin{aligned}
& \mathbf{H}=\sum_{i} h \boldsymbol{S}_{i}^{z}+\sum_{i} J_{1}\left(\boldsymbol{S}_{i}^{z} \mathbf{S}_{i+1}^{z}+\frac{1}{2}\left(\boldsymbol{S}_{i}^{+} \mathbf{S}_{i+1}^{-}+\boldsymbol{S}_{i}^{-} \boldsymbol{S}_{i+1}^{+}\right)\right) \\
& +\sum_{i} J_{2}\left(S_{i}^{z} \mathbf{S}_{i+2}^{z}+\frac{1}{2}\left(S_{i}^{+} \mathbf{S}_{i+2}^{-}+S_{i}^{-} \mathbf{S}_{i+2}^{+}\right)\right),
\end{aligned}
$$

where we added a magnetic field $h$. In the following we will treat the Majumdar-Ghosh model with $\mathrm{J}_{2}=\mathrm{J}_{1} / 2$.

When applying the cluster approximation to this zig-zag model, one has to choose a proper reference system. We will use a six-site cluster, which is already depicted in fig. 4.6 , because we found the best results for the spin chain with this choice. As for the previous model, it turns out that using the longitudinal $\mathrm{J}^{z}$ as variational parameter leads to worse results when compared to reference systems where it is kept fixed.

So the model has two potential terms for the variation, $\mathrm{J}_{1}^{\mathrm{t}}$ and $\mathrm{J}_{2}^{\mathrm{t}}$. This leaves several possibilities: First, one can vary both parameters simultaneously with the ratio $J_{1}^{t} / J_{2}^{t}=2$ intact. Second, the variation can be carried out using either $\mathrm{J}_{1}^{\mathrm{t}}$ or $\mathrm{J}_{2}^{\mathrm{t}}$ alone. As a third possibility, one can vary both independently. The latter choice would lead to a search in a two-dimensional parameter space. Running preliminary tests with this set-up, the results were not superior to the choice of varying only one of them while keeping the other fixed at the equivalent lattice quantity. Since the computational effort rises substantially for two parameters, we will only perform a one-dimensional search for stationary points. This leaves the first and second of the possibilities for the variation.

Interestingly, the best results are achieved when only $\mathrm{J}_{2}^{\mathrm{t}}$ is used while 


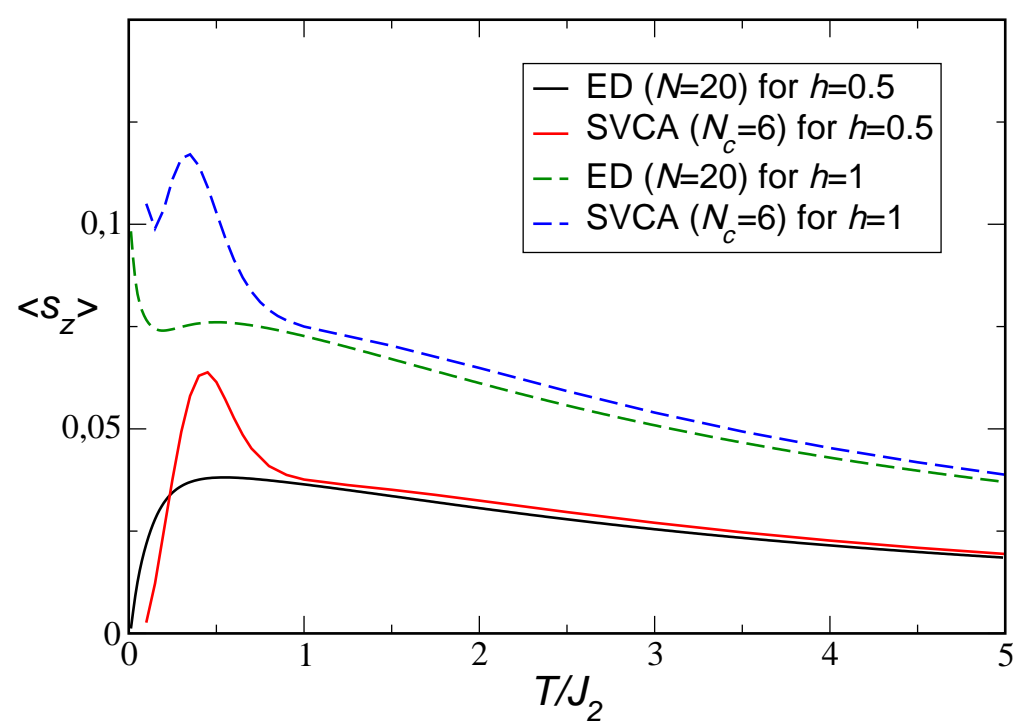

Figure 4.7: In the figure magnetization curves for the antiferromagnetic spin $1 / 2$ chain with frustrated interactions $J_{1}$ and $J_{2}$ are plotted for two different applied magnetic fields, $h=0.5 \mathrm{~J}_{2}$ and $\mathrm{h}=\mathrm{J}_{2}$. The results of SVCA computations for a six-site cluster and the transversal variational parameter $\mathrm{J}_{2}^{\mathrm{t}}$ are compared to ED calculations for 20 site lattices [HMHV06].

$\mathrm{J}_{1}^{\mathrm{t}}$ is fixed. The constant ratio variation leads to a magnetization which is consistently too high. This may seem unusual since the parameters of the reference system in the first case do not keep the frustration ratio of the original Majumdar-Ghosh model intact. Indeed, $\mathrm{J}_{1} / \mathrm{J}_{2}$ lies between approximately 1.6 and 2 in the range of the considered temperatures. One has to keep in mind, though, that the variational parameters do not have real physical meaning. They may give hints about the nature of the approximation, but only the value of the approximate SVCA free energy and subsequently the derived thermodynamical quantities have to be considered in the end. Nevertheless, the strong influence of the choice of the reference system is remarkable and would be an interesting endeavour for future research.

Fig. 4.7 shows SVCA results for the frustrated second-neighbour interaction spin- $1 / 2$ chain for two magnetic fields $h=0.5 J_{2}$ and $h=J_{2}$. The plotted magnetization curves were calculated for the aforementioned six-site cluster reference system with a variation of $J_{2}^{t}$ only.

They are compared to results of a 20 site cluster computed with exact diagonalization [HMHV06]. Several observations are noteworthy. First, the SVCA magnetization is larger than the ED calculation results. Moreover, the 'spiking' behaviour for low temperatures which was already seen for the simple spin chain in the sub-critical regime is also encountered here. However, the evaluation of the SVCA free energy is possible for much lower $T$. The fact that we do not show results for $T<0.1$ in the plots in fig. 4.7 has technical reasons. We can well assume that for the frustrated chain temperatures near $T=0$ are available for the SVCA. 
This result is noteworthy. It means that the breakdown of the theory indeed seems to be related to the long-range correlations present in the simple spin chain. This supports our interpretations from section 3.5 and 4.2. It provides credibility for the SVCA since the occurrence of a breakdown indeed seems to be related to actual physical processes in the original model. The fact that we do not encounter these for the frustrated chain with prevalent short-range correlations is encouraging for the future use of the SVCA. Namely, for treating systems with competing interactions.

On the negative side, we have to admit that the actual results are not as convincing as we hoped for. The spike of the magnetization for low temperatures is a persistent and irritating feature. It is at the moment not clear where this behaviour comes from and how it can be amended. We assume that it is related to the missing control over the magnetization of the reference system due to the lack of variational magnetic fields. This possible explanation was already discussed in the previous section.

To sum up, the treatment of the frustrated spin chain has shown that in principle the SVCA improves for systems with short-range interactions, as was hoped for. Yet, we encounter a systematic non-physical feature in the magnetization for low temperatures. This needs to be investigated and possibly amended for further applications of the SVCA. 



\section{CONCLUSION AND DISCUSSION}

The endeavour of this thesis was to find a variational cluster approach for spin systems, namely Heisenberg models or more generally Hamiltonians that feature interacting spin operators, and to use it for calculating their thermodynamical properties. The idea was to follow Potthoff's SEFA respectively VCA procedure for fermionic systems [Pot03a; Pot03b]. This has proven to be a delicate problem. The spin operators satisfy non-canonical commutation relations and form a Lie algebra. So the Baym-Kadanoff-Luttinger-Ward formalism of connecting a static thermodynamical potential to dynamic quantities like the one-particle Green function is not readily available. Apart from that, the SEFA relies on a proper separation of non-interacting part and interaction in the Hamiltonian. For the Heisenberg model no such division is straight-forward since it is inherently interacting. Moreover, the spin exchange is nonlocal. In the standard VCA approach for fermions no such interactions are allowed for.

Several approaches to overcome these problems and to establish a SEFAlike formalism for the Heisenberg model have been considered. Initially we followed three ideas which faced specific and severe difficulties. In chapter 2 we briefly discussed the perturbative spin diagram technique (section 2.2), transformations of the spin operators to bosonic or fermionic degrees of freedom (section 2.3) and a resolvent approach (section 2.4). None of these schemes met their expectations. Operator transformations only lead to additional approximations which can not be controlled in a reasonable way. For the resolvent method we found formal variational equations which are impossible to evaluate. Within the spin diagram technique we could not establish a suitable Baym-Kadanoff functional, yet elements of the method were used to finally introduce a cluster approximation.

This SVCA is the main result of the thesis and yields a new approach for Heisenberg spin systems. It is inspired by the SEFA for fermionic systems with local interactions [Pot03b], which has been subsequently extended to bosonic degrees of freedom [KD06] and to cluster approximations like Tong's EVCA for more complex models [Ton05]. The approach presented in chapter 3 of this thesis uses a path-integral representation for the partition function of a Heisenberg model. This yields the starting point to develop a theory that allows to devise a variational cluster method for spin models. 
In the original SEFA, the key object is the single-particle self-energy of fermions or bosons. It appears as the argument in the Potthoff functional (2.11) and approximations are applied directly to this quantity. For spin models a similar object is not available in a straight-forward way, but by means of the spin diagram technique we could identify the perturbatively defined Larkin self-energy [IS88; PSS69] as a suitable quantity for this step in the theory.

With this self-energy we could define a generalized spin Luttinger-Ward functional (3.23) by means of functional analysis. We finally derived a set of variational equations (3.32), which we called SVCA equations. These give approximate solutions for the properties of spin systems: By introducing certain solvable cluster reference systems one essentially restricts the domain of the spin self-energy. Subsequently, one searches for stationary points of the free energy functional $F_{S \vee C A}$. This variational principle yields approximations on the domain given by the chosen reference system. These values of the free energy can then be used as a starting point to derive other thermodynamical quantities like the specific heat for the spin model under consideration.

To make use of the SVCA equations and to find stationary points, one needs to evaluate them for a certain reference system. An explicit way to proceed is given in section 3.4.

By the scheme presented in chapter 3 , we introduced a new and working variational cluster approach to the thermodynamics of Heisenberg spin systems.

Yet, one encounters certain specific problems for the SVCA which have to be addressed. The way the method is presently formulated, one important limitation is that we cannot use local magnetic fields as variational parameters. Such terms appear routinely in fermionic or bosonic theories and are used to control thermodynamic consistency between reference cluster and physical lattice. Moreover, additional Weiß fields have proven to be effective to describe certain phases and phase transitions [Pot12a].

A local field that could be incorporated directly in the SVCA method, though, is an easy-axis term of the form $a\left(\mathbf{S}^{z}\right)^{2}$. However, for the spin $1 / 2$-systems which have been treated thus far, the term becomes a/4 and its application did not give any improvement of the approximation. For future work, it would be important to apply the SVCA to models where such easy-axis terms naturally appear, so one could investigate if local variational parameters of that form are useful at all.

However, the SVCA should eventually be extended to implement magnetic fields. Apart from the aforementioned applications this might be helpful in treating certain limitations of the method. Such variational parameters are not available due to the derivation and the structure of the SVCA equations presented in chapter 3. A possible solution is discussed in appendix D. 
What is used as standard variational parameters in the SVCA are the interactions $\mathrm{J}$, or more specifically the transversal $\mathrm{J}^{\mathrm{t}}$ since the longitudinal $\mathrm{J}^{z}$ often leads to worse approximations.

The fact that we solely use $\mathrm{J}^{\mathrm{t}}$ in the SVCA marks a difference to the standard VCA for bosonic and fermionic systems. Here, the formal equivalent to the $J$ in the theory are the hopping parameters $t$. Yet they are often held constant during the search for the stationary points while local fields are varied [Pot12a]. The SVCA until now uses predominantly non-local parameters for finding solutions.

A second interesting issue with the SVCA stems from the fact that due to its analytical structure it can fail to find physical solutions in certain situations. One could interpret this breakdown of the theory as a signal for a phase transition, although it needs not be one of the real model. The way in which these breakdowns appear makes such an interpretation very tempting. A more conservative point of view is that such a breakdown indicates that the present choice of the reference cluster system, respectively the set of variational parameters, is not able to treat certain properties properly like an increase of correlation lengths. If this is the case, one should be able to see an improvement by choosing larger clusters - as for the spin chain in section 4.2 - or variational parameters that are better suited for the problem at hand.

Nevertheless, a breakdown of the SVCA could be used to monitor important changes in the physical state of the spin model. For future approaches using the SVCA, it could be of some importance to search systematically for these breakdowns. Generally, it would be favourable to better understand their nature and the way they appear in the approximation.

To test the SVCA in chapter 4 we studied the antiferromagnetic Heisenberg $S=1 / 2$ chain in a magnetic field where we could compare our results for thermodynamical quantities with the exact Bethe ansatz solutions.

Initially three reference systems - full Heisenberg interaction, transversal and longitudinal interaction separately and transversal interaction only as variational parameters - with different cluster sizes were used for several magnetic fields to apply the SVCA to the model. It turned out that the longitudinal interaction is not suited as a variational parameter. We discussed several conceivable reasons for this result. Possibly, it is related to the fact, which was already mentioned earlier in this chapter, that one cannot use a local field in the cluster Hamiltonian as variational parameter. So we cannot control the local magnetization explicitly which might lead to a compensating behaviour of the longitudinal variational interaction. Nevertheless, it is not entirely clear why using $\mathrm{J}^{z}$ in the variation leads to worse results. Further work on different models has to be done to investigate this problem.

The solutions found with the SVCA using only transversal parameters are good above the critical point $h=2 J$. This is a first evidence which 
supports our approximation scheme. Below the critical point we find the aforementioned breakdowns for certain quantified temperatures. These signal that the cluster approximation is not able to cope with the increasing long-range correlations. The results also seem to be less reliable in the sub-critical regime in general. Yet, we also find that they in principle improve with cluster size which is to be expected.

This application to the spin chain provided a proof of principle that the SVCA leads to reasonable approximate results for Heisenberg models. Here, we could concentrate on understanding the analytical structure of the SVCA equations and get valuable insight into the method.

As discussed before, we attributed the breakdown of the approximation at low temperatures to increasing long-range correlations. To further test the approximation, we treated the antiferromagnetic zig-zagladder in section 4.3. In this model long-range correlations play a lesser role than for the antiferromagnetic chain due to frustration effects. However, the results for low T still show unusual 'spiking' behaviour. This can be interpreted in the sense that the approximation still copes with long-range interactions for small cluster sizes.

Yet, no breakdown occurs and the SVCA equations can be evaluated down to $T \approx 0$. Hence, the approximation is not signalling a pseudo phase transition as for the spin chain, which is an improvement. Indeed, the results show that the SVCA could be more useful for frustrated spin systems. It was one of the goals of establishing a spin VCA to treat such models. Nevertheless, the actual numerical results are less convincing. The magnetization is too high for low temperatures. This consistent behaviour might also be associated with the missing control due to the fixed magnetic fields.

One additional point needs to be added regarding the application of the SVCA to certain spin models. The derivation of the SVCA was based on a Heisenberg Hamiltonian with an applied magnetic field. Naturally, one is often interested in systems without such additional terms, for example when treating models of real physical materials. It turns out for the cases tested so far that the SVCA computations break down in the sense discussed above when the field is set to zero. Yet, if a very small regularizing term is added one finds proper results.

This can be understood in the following way. The approximation has been derived using a formalism where the $\mathrm{SU}(2)$ symmetry is broken. It is specifically based on the assumption that the transversal and longitudinal terms can be treated differently. If the external field is set to zero for an isotropic Hamiltonian, this distinction is redundant. To apply the method, the SVCA free energy should then be evaluated only using the longitudinal formalism from 3.4. We have already seen before that this part of the variation is less reliable. Moreover, the symmetric case forbids the identification of the Larkin self-energy within the derivation of the approximation.

This makes it reasonable that a small regularizing field is needed to 
lift the symmetry of the model. The fact that so far all reasonable reference systems lead to non-equivalent longitudinal and transversal interactions supports this step.

Further investigations with larger clusters are needed to confirm and extend our observations. However, to achieve this goal one will need more efficient algorithms to treat open spin clusters. Up to now we only used a full exact diagonalization. Examples for more powerful cluster solvers include the density-matrix renormalization group [Sch05], the matrix product state method [PWE10] or quantum Monte-Carlo [San10]. For the latter one would have to keep the sign problem under control, which for example was recently done in computations on frustrated spin ladder systems [ADP15; $\left.\mathrm{Hon}^{+} 16\right]$. One could possibly also employ different algorithms to evaluate the SVCA equations, which might be better suited for larger clusters. The way presented in 3.4 is but one of several possibilities [Sen08].

One has to keep in mind that we are working with finite temperatures and thus have to work with the full Hilbert space of the Hamiltonian. The SVCA equations deal with matrices of a rank equal to the excitations in the system, which usually are of the order of number of states squared - though less than that because some transition elements have zero weight. Nevertheless, the computation becomes costly very fast for increasing cluster size. Here, some scheme to reduce the number of states that have to be taken into account seems inevitable. One natural candidate is the Lanczos method [Lan50; Wil65].

The SVCA should also be tested for systems with spin $S>1 / 2$. This of course leads to Hilbert spaces of higher dimensions which again increases the numerical effort. So for all these future applications, the need for a more efficient numerical treatment is evident.

Generally, applying the SVCA to an antiferromagnetic spin chain and the zig-zag ladder system can only be the first step. To really establish its usefulness one should also treat other models, especially proper ladders or two-dimensional lattices with frustrating interactions. Our present results indicate that the SVCA in principle improves when long-ranged correlations are suppressed. This observation makes it particularly interesting to apply the approximation to frustrated spin systems, where short-ranged correlations often dominate for low temperatures. For instance, this condition is met in several two-dimensional antiferromagnetic spin lattices [RSH04].

Yet, for two-dimensional models with frustration the SVCA equations are more delicate and time-consuming to solve. The clusters are supposedly larger and the evaluation includes a two-dimensional mesh in the k-space that has to be summed over (see section 3.4 and appendix C). Additionally, frustration is sometimes realized by two competing interactions which could demand two variational parameters. This means that one has to search for a stationary point in a two-dimensional space. 
Of course, this proves to be numerically challenging for the exact diagonalization approaches we took. Again, we need more sophisticated numerical methods to solve the SVCA equations and find specific stationary points for two-dimensional spin systems.

To conclude, we presented a novel variational cluster approach for spin systems in this thesis that in principle allows for suitable treatment of Heisenberg models. We were able to test it on chain models and to observe several important features: In regions where short-ranged correlations dominate we could find good agreement with the exact results. Generally, the approximation improves with cluster size. As expected, the results are less accurate in the presence of long-range order and for low temperatures. In certain such regions the approximation breaks down altogether, which is an interesting feature since it might signal important changes in the spin system. There are still open questions which have to be investigated further, using more efficient numerical methods.

From a conceptual point of view, the most urgent problem is the integration of local fields into the SVCA. A first approach which remains to be fully explored is discussed in appendix D. The possibility of local variational parameters could amend certain issues with the magnetization and the longitudinal interactions. It also could allow for an improved treatment of different phases in certain spin models with local order parameters, like it is the case for the standard fermionic SEFA. The SVCA proposed here in this thesis is a promising starting point, but some work has still to be done to explore its full potential. 


\section{SPIN-COHERENT STATES AND PATH INTEGRALS}

In this appendix the spin path integral will be introduced. The derivation is based on the work of Fradkin and Stone [Fra91; FS88], where further details can be found. The spin path integral can be constructed by means of spin-coherent states, which were introduced and reviewed by Perelomov [Per86]. As a first step these states need to be defined.

\section{A.1 SPIN-COHERENT STATES}

Consider a single spin with the usual Hilbert space spanned by the vectors $|s, m\rangle$. The quantum numbers are given by the eigenvalue equations $\overrightarrow{\mathbf{S}}^{2}|s, \mathrm{~m}\rangle=\mathrm{s}(\mathrm{s}+1)|\mathrm{s}, \mathrm{m}\rangle$ and $\mathbf{S}^{z}|s, \mathrm{~m}\rangle=\mathrm{m}|\mathrm{s}, \mathrm{m}\rangle$, so the quantization axis is in $z$-direction. The states $|s, \pm s\rangle$ with maximal magnetic quantum numbers $m$ can be seen as the ones that are closest to classical states in the sense that for them the total spin operator $\overrightarrow{\mathbf{S}}=\left(\mathbf{S}^{x}, \mathbf{S}^{y}, \mathbf{S}^{z}\right)$ has the lowest possible dispersion [Per86; CR12]. One of these is preferably taken as the reference state to introduce spin-coherent states. Without loss of generality in the following the vector $|s, s\rangle$ is chosen.

Suppose $\vec{n}=(\sin \theta \cos \varphi, \sin \theta \sin \varphi, \cos \theta)$ is a vector along the unit sphere, with $0 \leqslant \theta \leqslant \pi$ and $0 \leqslant \varphi<2 \pi$, and $\vec{n}_{0}=(0,0,1)$ points in the direction of the quantization axis. Then a spin-coherent state of $S U(2)$ is introduced as:

$$
\begin{aligned}
|\overrightarrow{\mathrm{n}}\rangle & =\mathbf{D}(\overrightarrow{\mathrm{n}})|s, s\rangle \\
& =\exp \left(i \theta \frac{\overrightarrow{\mathrm{n}} \times \overrightarrow{\mathrm{n}_{0}}}{\left|\overrightarrow{\mathrm{n}} \times \overrightarrow{\mathrm{n}_{0}}\right|} \overrightarrow{\mathbf{s}}\right)|s, s\rangle \\
& =\exp \left(i \theta\left(\sin \varphi \mathbf{S}^{x}-\cos \varphi S^{y}\right)\right)|s, s\rangle .
\end{aligned}
$$

The operator $\mathbf{D}(\overrightarrow{\mathrm{n}})$ rotates by the angle $\theta$ about an axis defined through the vector $\left(\vec{n} \times \overrightarrow{n_{0}}\right) /\left|\vec{n} \times \overrightarrow{n_{0}}\right|=(\sin \varphi,-\cos \varphi, 0)$. Each spin-coherent state $|\vec{n}\rangle$ given by (A.1) is parametrized by a certain $\vec{n}$ and thus corresponds to a point on the unit sphere. So essentially the phase space of a classical spin is represented by the rotation.

The product of two operators $\mathbf{D}$ with respect to different vectors is given by:

$$
\mathbf{D}\left(\overrightarrow{\mathrm{n}}_{1}\right) \mathbf{D}\left(\overrightarrow{\mathrm{n}}_{2}\right)=\mathbf{D}\left(\overrightarrow{\mathrm{n}}_{3}\right) \exp \left(-i \Phi\left(\overrightarrow{\mathrm{n}}_{1}, \overrightarrow{\mathrm{n}}_{2}\right) \boldsymbol{S}^{z}\right)
$$


where $\vec{n}_{3}=R_{\vec{n}_{1}} \vec{n}_{2}$. Here, $R_{\vec{n}_{1}}$ is the geometrical rotation associated with the operator $D\left(\vec{n}_{1}\right)$, so $\vec{n}_{3}$ is derived by rotating the vector $\vec{n}_{0}$ twice. As can be seen from the second term on the right hand side of (A.2) the operators D do not form a group. The angle $\Phi\left(\vec{n}_{1}, \vec{n}_{2}\right)$ is the oriented area of the geodesic triangle on the sphere between the vectors $\vec{n}_{0}, \vec{n}_{1}$ and $\vec{n}_{2}$. From (A.2) follows that a rotation operator transforms a spincoherent state into another one, up to a phase factor:

$$
\mathbf{D}\left(\vec{n}_{1}\right)\left|\vec{n}_{2}\right\rangle=\mathbf{D}\left(\vec{n}_{1}\right) \mathbf{D}\left(\vec{n}_{2}\right)|s, s\rangle=\exp \left(-i s \Phi\left(\vec{n}_{1}, \vec{n}_{2}\right)\right)\left|\vec{n}_{3}\right\rangle .
$$

The area $\Phi\left(\vec{n}_{1}, \vec{n}_{2}\right)$ of the spherical triangle is not unique. One can arbitrarily choose between two possibilities since the sphere is a closed manifold. The difference of the areas is $4 \pi$. This arbitrary choice must not have any physical consequences for the spin-coherent states, for example in (A.3). So it is mandatory that $\exp (-i s 4 \pi)=1$ which holds for integer and half-integer spin quantum numbers. The requirement can be seen within this formulation as the reason for the quantization of the spins.

Equations (A.2) respectively (A.3) lead directly to the scalar product:

$$
\left\langle\vec{n}_{1} \mid \vec{n}_{2}\right\rangle=\exp \left(-i s \Phi\left(\vec{n}_{1}, \vec{n}_{2}\right)\right)\left(\frac{1+\vec{n}_{1} \cdot \vec{n}_{2}}{2}\right)^{s}
$$

So the spin-coherent states are not orthogonal and form an over-complete basis of the spin Hilbert space. The resolution of identity can be given as:

$$
\int \mathrm{d} \mu(\overrightarrow{\mathrm{n}})|\overrightarrow{\mathrm{n}}\rangle\langle\overrightarrow{\mathrm{n}}|=\mathbb{1},
$$

with the integration measure:

$$
\mathrm{d} \mu(\overrightarrow{\mathrm{n}})=\frac{2 \mathrm{~s}+1}{4 \pi} \mathrm{d}^{3} \mathrm{n} \delta\left(\overrightarrow{\mathrm{n}}^{2}-1\right) .
$$

Proofs for equations (A.2)-(A.6) and further discussion on the spin coherent states can be found in chapter 7 of the book by Combescure and Robert [CR12].

The diagonal matrix elements of the spin operators in the basis of coherent states will be needed for the derivation of the spin path integral and thus computed explicitly in the following. Using the above definition (A.1) one can write for $\mathbf{S}^{z}$ :

$$
\begin{aligned}
& \left\langle\vec{n}\left|S^{z}\right| \vec{n}\right\rangle=\left\langle s, s\left|D^{\dagger}(\vec{n}) S^{z} \mathbf{D}(\vec{n})\right| s, s\right\rangle \\
& =\langle s, s| \exp \left(-i \theta\left(\sin \varphi S^{x}-\cos \varphi S^{y}\right)\right) \\
& \text {. } \boldsymbol{S}^{z} \exp \left(i \theta\left(\sin \varphi \boldsymbol{S}^{x}-\cos \varphi \boldsymbol{S}^{y}\right)\right)|s, s\rangle
\end{aligned}
$$

To evaluate this expectation value Hadamard's formula is used [Mil73]:

$$
e^{-\mathbf{A}} \mathbf{B} e^{\mathbf{A}}=\mathbf{B}+[\mathbf{B}, \mathbf{A}]+\frac{1}{2 !}[[\mathbf{B}, \mathbf{A}], \mathbf{A}]+\frac{1}{3 !}[[[\mathbf{B}, \mathbf{A}], \mathbf{A}], \mathbf{A}]+\ldots
$$


As a result of this expansion, the operators in the second line of (A.7) can be written as:

$$
\begin{aligned}
\mathbf{D}^{\dagger}(\overrightarrow{\mathrm{n}}) \mathbf{S}^{z} \mathbf{D}(\overrightarrow{\mathrm{n}})= & \mathbf{S}^{z}-\theta\left(\cos \varphi \mathbf{S}^{\boldsymbol{x}}+\sin \varphi \mathbf{S}^{y}\right) \\
& \quad-\frac{\theta^{2}}{2} \mathbf{S}^{z}+\frac{\theta^{3}}{6}\left(\cos \varphi \mathbf{S}^{x}+\sin \varphi \mathbf{S}^{y}\right)+\ldots \\
= & \cos \theta \mathbf{S}^{z}-\sin \theta\left(\cos \varphi \mathbf{S}^{x}+\sin \varphi \mathbf{S}^{y}\right)
\end{aligned}
$$

where the usual spin commutation relations $\left[\boldsymbol{S}^{i}, \boldsymbol{S}^{j}\right]=i \epsilon_{i j k} \boldsymbol{S}^{k}$ and the expansion of sine and cosine were used. Keeping in mind that $\boldsymbol{S}^{\boldsymbol{x}}=$ $\frac{\mathbf{S}^{+}+\mathbf{S}^{-}}{2}$ and $\mathbf{S}^{y}=\frac{\mathbf{S}^{+}-\mathbf{S}^{-}}{2 i}$ one can easily see that the matrix element (A.7) becomes:

$$
\left\langle\vec{n}\left|\mathbf{S}^{\mathcal{z}}\right| \vec{n}\right\rangle=\cos \theta\left\langle s, s\left|\mathbf{S}^{z}\right| s, s\right\rangle=s \cos \theta=s n_{z} .
$$

For the other two spin components a similar computation delivers:

$$
\begin{aligned}
& \mathbf{D}^{\dagger}(\vec{n}) \mathbf{S}^{x} \mathbf{D}(\vec{n})=\sin \theta \cos \varphi \mathbf{S}^{z}+(\cos \theta-1) \sin \varphi \cos \varphi \mathbf{S}^{y} \\
& +\left(\sin ^{2} \varphi+\cos ^{2} \varphi \cos \theta\right) \mathbf{S}^{\chi} \\
& \left\langle\vec{n}\left|S^{\chi}\right| \vec{n}\right\rangle=\sin \theta \cos \varphi\left\langle s, s\left|S^{z}\right| s, s\right\rangle \\
& =s \sin \theta \cos \varphi=s n_{x} \text {, } \\
& \mathbf{D}^{\dagger}(\overrightarrow{\mathrm{n}}) \boldsymbol{S}^{y} \mathbf{D}(\overrightarrow{\mathrm{n}})=\sin \theta \sin \varphi \boldsymbol{S}^{z}+(\cos \theta-1) \sin \varphi \cos \varphi \boldsymbol{S}^{x} \\
& +\left(\cos ^{2} \varphi+\sin ^{2} \varphi \cos \theta\right) S^{y} \\
& \left\langle\vec{n}\left|S^{y}\right| \vec{n}\right\rangle=\sin \theta \sin \varphi\left\langle s, s\left|S^{z}\right| s, s\right\rangle \\
& =s \sin \theta \sin \varphi=s n_{y} \text {. }
\end{aligned}
$$

In a compact notation equations (A.10)-(A.12) give the important result:

$$
\langle\vec{n}|\vec{S}| \vec{n}\rangle=s \vec{n} .
$$

So the expectation value of the total spin operator in a coherent state $|\vec{n}\rangle$ is a vector of length $s$ in the direction $\vec{n}$. This will be vital for the derivation of a spin path integral in the next section. 


\section{A.2 THE SPIN PATH INTEGRAL}

In the following the path integral for a spin model will be derived. Further discussion can be found in the books by Negele and Orland [NO88] and by Fradkin [Fra91]. In a first step a simple model of one spin $\overrightarrow{\boldsymbol{S}}$ with a Hamiltonian $\mathbf{H}(\overrightarrow{\mathbf{S}})$ is considered. The Trotter-Suzuki decomposition is used on the partition function of the system:

$$
\begin{aligned}
Z & =\operatorname{Tr} e^{-\beta H}=\lim _{N_{t} \rightarrow \infty, \delta t \rightarrow 0} \operatorname{Tr}\left(e^{-\delta t H}\right)^{N_{t}} \\
& =\lim _{t \rightarrow \infty, \delta t \rightarrow 0} \int d \mu(\vec{n})\left\langle\vec{n}\left|\left(e^{-\delta t H}\right)^{N_{t}}\right| \vec{n}\right\rangle,
\end{aligned}
$$

with $\delta \mathrm{t} \cdot \mathrm{N}_{\mathrm{t}}=\beta$ held constant. With this the imaginary-time interval $[0, \beta]$ is divided into $N_{t}$ steps of length $\delta t$. In the second line the trace is displayed using the spin-coherent states defined in the previous section, with the integration measure $d \mu(\vec{n})$ from (A.6). Now the identity (A.5) is inserted $\left(N_{t}-1\right)$ times between the exponential operators:

$$
Z=\lim _{N_{t} \rightarrow \infty, \delta t \rightarrow 0}\left(\prod_{j=1}^{N_{t}} \int d \mu\left(\vec{n}\left(t_{j}\right)\right)\right)\left(\prod_{j=1}^{N_{t}}\left\langle\vec{n}\left(t_{j}\right)\left|e^{-\delta t H}\right| \vec{n}\left(t_{j+1}\right)\right\rangle\right)
$$

where the $t_{j}$ are intermediate imaginary times with periodic boundary conditions and thus the $|\vec{n}\rangle$ from (A.14) are taken to be the initial and final states $\left|\vec{n}\left(t_{1}\right)\right\rangle=\left|\vec{n}\left(t_{N_{t}+1}\right)\right\rangle$. The integrations can be seen to go over all sufficiently smooth closed paths along the unit sphere. For the integrals a formal representation is used in the following:

$$
\int \mathcal{D} \vec{n}=\prod_{j=1}^{N_{t}} \int \mathrm{d} \mu\left(\vec{n}\left(t_{j}\right)\right) .
$$

The exponential operator in (A.15) is expanded in the given limit up to order $\mathrm{O}(\delta \mathrm{t})$ :

$$
\begin{aligned}
Z & =\lim _{N_{t} \rightarrow \infty, \delta t \rightarrow 0} \int \mathcal{D} \vec{n} \prod_{j=1}^{N_{t}}\left[\left\langle\vec{n}\left(t_{j}\right) \mid \vec{n}\left(t_{j+1}\right)\right\rangle-\delta t\left\langle\vec{n}\left(t_{j}\right)|\mathbf{H}| \vec{n}\left(t_{j+1}\right)\right\rangle\right] \\
& =\lim _{N_{t} \rightarrow \infty, \delta t \rightarrow 0} \int \mathcal{D} \vec{n} \prod_{j=1}^{N_{t}}\left\langle\vec{n}\left(t_{j}\right) \mid \vec{n}\left(t_{j+1}\right)\right\rangle\left[1-\delta t\left\langle\vec{n}\left(t_{j}\right)|\mathbf{H}| \vec{n}\left(t_{j}\right)\right\rangle\right] \\
& =\lim _{N_{t} \rightarrow \infty, \delta t \rightarrow 0} \int \mathcal{D} \vec{n} \prod_{j=1}^{N_{t}}\left\langle\vec{n}\left(t_{j}\right) \mid \vec{n}\left(t_{j+1}\right)\right\rangle \exp \left(-\delta t\left\langle\vec{n}\left(t_{j}\right)|\mathbf{H}| \vec{n}\left(t_{j}\right)\right\rangle\right)
\end{aligned}
$$

In the second line the additional approximation was used:

$$
\begin{aligned}
\frac{\left\langle\vec{n}\left(t_{j}\right)|H| \vec{n}\left(t_{j+1}\right)\right\rangle}{\left\langle\vec{n}\left(t_{j}\right) \mid \vec{n}\left(t_{j+1}\right)\right\rangle} & =\frac{\left\langle\vec{n}\left(t_{j}\right)|H| \vec{n}\left(t_{j}+\delta t\right)\right\rangle}{\left\langle\vec{n}\left(t_{j}\right) \mid \vec{n}\left(t_{j}+\delta t\right)\right\rangle} \\
& =\left\langle\vec{n}\left(t_{j}\right)|H| \vec{n}\left(t_{j}\right)\right\rangle+O(\delta t) .
\end{aligned}
$$


For $\left\langle\vec{n}\left(t_{j}\right) \mid \vec{n}\left(t_{j+1}\right)\right\rangle$ the inner product (A.4) is applied:

$$
\left\langle\vec{n}\left(t_{j}\right) \mid \vec{n}\left(t_{j+1}\right)\right\rangle=\exp \left(-i s \Phi\left(\vec{n}\left(t_{j}\right), \vec{n}\left(t_{j+1}\right)\right)\right)\left(\frac{1+\vec{n}\left(t_{j}\right) \cdot \vec{n}\left(t_{j+1}\right)}{2}\right)^{s} .
$$

With this the partition function of the spin can be written as:

$$
\begin{aligned}
Z= & \lim _{N_{t} \rightarrow \infty, \delta t \rightarrow 0} \int \mathcal{D} \vec{n} e^{-S[\vec{n}]} \\
S[\vec{n}]= & i s \sum_{j=1}^{N_{t}} \Phi\left(\vec{n}\left(t_{j}\right), \vec{n}\left(t_{j+1}\right)\right)-s \sum_{j=1}^{N_{t}} \ln \left(\frac{1+\vec{n}\left(t_{j}\right) \cdot \vec{n}\left(t_{j+1}\right)}{2}\right) \\
& +\sum_{j=1}^{N_{t}} \delta t\left\langle\vec{n}\left(t_{j}\right)|H| \vec{n}\left(t_{j}\right)\right\rangle
\end{aligned}
$$

The functions $\Phi$ that appear in the first term of the action is the spherical triangular area defined by the three vectors $\vec{n}\left(t_{j}\right), \vec{n}\left(t_{j+1}\right)$ and $\vec{n}_{0}$. The sum over these give an area bounded by some closed path along the sphere. Expressions of this sort are called Wess-Zumino terms $S_{W Z}$ [Fra91].

In the logarithm under the second sum from (A.21) the vector $\vec{n}\left(t_{j+1}\right)=$ $\vec{n}\left(t_{j}+\delta t\right)$ can be Taylor expanded to arrive at:

$$
\ln \left(1+\frac{\delta t^{2}}{4} \vec{n}\left(t_{j}\right) \cdot\left(\partial_{t}^{2} \vec{n}\left(t_{j}\right)\right)\right) \simeq \frac{\delta t^{2}}{4} \vec{n}\left(t_{j}\right) \cdot\left(\partial_{t}^{2} \vec{n}\left(t_{j}\right)\right),
$$

where the first order in $\delta$ t vanishes due to $\vec{n}\left(t_{j}\right) \cdot\left(\partial_{t} \vec{n}\left(t_{j}\right)\right)=0$.

Without loss of generality, the single spin Hamiltonian is chosen to be $\mathbf{H}=\overrightarrow{\mathrm{h}} \cdot \overrightarrow{\mathbf{S}}$ with a magnetic field in arbitrary direction. Using (A.13) the expectation value in the third term of the action (A.21) becomes:

$$
\left\langle\vec{n}\left(t_{j}\right)|\mathbf{H}| \vec{n}\left(t_{j}\right)\right\rangle=s \vec{h} \cdot \vec{n}\left(t_{j}\right) .
$$

Now one can perform the naive continuum limit $N_{t} \rightarrow \infty$ and $\delta t \rightarrow 0$ for the spin action (A.21):

$$
S[\vec{n}]=i s S_{W Z}(\vec{n})+\frac{s \delta t}{4} \int_{0}^{\beta} d t\left(\partial_{t} \vec{n}(t)\right) \cdot\left(\partial_{t} \vec{n}(t)\right)+s \int_{0}^{\beta} d t \vec{h} \cdot \vec{n}(t)
$$

where in the second term partial integration was applied and again $\vec{n}\left(t_{j}\right) \cdot\left(\partial_{t} \vec{n}\left(t_{j}\right)\right)=0$. One can find an interesting mechanical analogy for this action. A similar result is obtained for a charged particle moving along the sphere under the influence of a magnetic monopole positioned at its centre [Sto86]. In the present case the mass of that particle is $s \delta t / 2$. So in the given limit $\delta t \rightarrow 0$ the quasi-classical correspondence for a spin would be a massless particle that is moving on a sphere with a vector potential attached, which is a reasonable result [Fra91]. For any practicable purposes the middle term of action (A.24) can be set to zero. 
The extension of the spin path integral to a system of $\mathrm{N}$ spins on an arbitrary lattice is straight forward. The Hilbert space for such a model is the $\mathrm{N}$-fold tensor product of the single spin Hilbert space. One can define the spin-coherent state of the full system as:

$$
|\otimes(\vec{n})\rangle=\bigotimes_{i=1}^{N}\left|\vec{n}_{i}\right\rangle .
$$

The resolution of the identity is given by:

$$
\int \prod_{i=1}^{N} \mathrm{~d} \mu\left(\vec{n}_{i}\right)|\otimes(\vec{n})\rangle\langle\otimes(\vec{n})|=\mathbb{1},
$$

with the individual integration measures $d \mu\left(\vec{n}_{i}\right)$ taken from (A.6). Now the partition function of an $\mathrm{N}$-spin Hamiltonian can be treated similar to equations (A.14)-(A.22) for the single spin system to arrive at the path integral representation:

$$
\begin{aligned}
Z= & \int \mathcal{D} \vec{n} e^{-S[\vec{n}]}, \\
S[\vec{n}]= & \text { is } \sum_{i=1}^{N} S_{W Z}\left(\vec{n}_{i}\right)+\frac{s \delta t}{4} \sum_{i=1}^{N} \int_{0}^{\beta} d t\left(\partial_{t} \vec{n}_{i}(t)\right)^{2} \\
& +\int_{0}^{\beta} d t\langle\otimes(\vec{n}(t))|H| \otimes(\vec{n}(t))\rangle,
\end{aligned}
$$

where the integration $\int \mathcal{D} \vec{n}$ is an abbreviation in the sense of (A.16) for $\mathrm{N}$ spins. Through the Wess-Zumino term in the action a phase factor is accumulated on a closed path along the unit sphere. It arises during a cyclic adiabatic process in the phase space of the system. So in essence the first term in (A.28) constitutes the Berry phase of the spin model [Ber84; Fra91]. It is not dependent on the details of the Hamiltonian $\mathbf{H}$ but rather on the geometrical aspects of the closed paths of the $\mathrm{N}$ spins in the system. In the following the Berry phase in the action will be denoted with $B[\vec{n}]$. As in the case of a single spin the second term in (A.28) can be neglected.

The last expression in the action of the path integral depends on the specific Hamiltonian of the system. The central relation to evaluate this expectation value is equation (A.13) $\langle\vec{n}|\vec{S}| \vec{n}\rangle=s \vec{n}$. Let $\vec{S}_{i}$ be a spin operator acting on the sub Hilbert space of the $i$-th spin. Then for an isotropic Heisenberg model one finds:

$$
\begin{aligned}
\langle\otimes(\vec{n}(t))|\mathbf{H}| \otimes(\vec{n}(t))\rangle & =\left\langle\otimes(\vec{n}(t))\left|\sum_{i, j} J_{i j} \vec{S}_{i} \vec{S}_{j}\right| \otimes(\vec{n}(t))\right\rangle \\
& =\sum_{i, j} J_{i j}\left\langle\vec{n}_{i}(t)\left|\vec{S}_{i}\right| \vec{n}_{i}(t)\right\rangle\left\langle\vec{n}_{j}(t)\left|\vec{S}_{j}\right| \vec{n}_{j}(t)\right\rangle \\
& =\sum_{i, j} J_{i j} s^{2} \vec{n}_{i}(t) \cdot \vec{n}_{j}(t)
\end{aligned}
$$


So the action $S_{H M}$ of the path integral representation for this Hamiltonian reads:

$$
S_{H M}[\vec{n}]=B[\vec{n}]+\int_{0}^{\beta} d t \sum_{i, j} J_{i j} s^{2} \vec{n}_{i}(t) \cdot \vec{n}_{j}(t) .
$$

The spin path integral for the anisotropic Heisenberg system with an applied magnetic field is similarly constructed with:

$$
\begin{aligned}
& \begin{aligned}
\langle\otimes(\vec{n}(t))|\mathbf{H}| \otimes(\vec{n}(t))\rangle & = \\
=\langle\otimes(\vec{n}(t))|\left(\sum_{i} h S_{i}^{z}\right. & +\sum_{i, j} J_{i j}^{z z} S_{i}^{z} S_{j}^{z} \\
& \left.+\sum_{i, j} J_{i j}^{-+}\left[S_{i}^{x} S_{j}^{x}+S_{i}^{y} S_{j}^{y}\right]\right)|\otimes(\vec{n}(t))\rangle \\
=s \sum_{i} h n_{i}^{z}(t)+s^{2} \sum_{i, j}\left(J_{i j}^{z z} n_{i}^{z}(t) n_{j}^{z}(t)\right. & \left.\quad J_{i j}^{-+}+\left[n_{i}^{x}(t) n_{j}^{x}(t)+n_{i}^{y}(t) n_{j}^{y}(t)\right]\right),
\end{aligned}
\end{aligned}
$$

where the matrix elements (A.10)-(A.13) were used. For convenience the variables $\vec{s}_{i}=s \vec{n}_{i}$ are introduced to write the action of the path integral as:

$$
\begin{aligned}
& S_{\text {AHM }}[\vec{s}]=\mathrm{B}[\vec{s}]+\int_{0}^{\beta} \mathrm{d} t \sum_{i} h s_{i}^{z}(t) \\
& +\int_{0}^{\beta} \mathrm{dt} \sum_{i, j}\left(J_{i j}^{z z} s_{i}^{z}(t) s_{j}^{z}(t)\right. \\
& \left.\quad+J_{i j}^{-+}\left[s_{i}^{x}(t) s_{j}^{x}(t)+s_{i}^{y}(t) s_{j}^{y}(t)\right]\right) .
\end{aligned}
$$





\section{SINGULAR MATRIX OF CORRELATION FUNCTIONS}

This appendix is concerned with the matrix of temperature correlation functions of self-adjoint operators, indexed by spatial coordinates. It will be shown that it is singular under certain circumstances, so the inverse as well as the logarithm does not exist. Such correlation matrices and the respective functions do appear in the SVCA approach presented in this thesis. In the appendix we will first prove the property and then discuss its implications.

\section{B.1 THE SINGULAR CORRELATION MATRIX}

Suppose we have a lattice of $N$ sites and some self-adjoint operator $\boldsymbol{A}_{i}$ acting on site i. $\boldsymbol{A}_{i}$ can for example be a particle number operator or $\boldsymbol{S}_{z}$. Further suppose a Hamiltonian $\mathrm{H}$ that acts on this system and conserves the total quantity $\sum_{i} \boldsymbol{A}_{i}$. This means that both are diagonalizable by the same set of eigenstates $|n\rangle$, which form a basis of rank $K$ of the relevant Hilbert space.

We will now discuss the temperature correlation function $\Pi_{i j}(\tau)=$ $\left\langle\boldsymbol{A}_{\boldsymbol{i}}(\boldsymbol{\tau}) \boldsymbol{A}_{\mathbf{j}}(0)\right\rangle$. In Matsubara frequency space it can be written using the Lehman representation:

$$
\Pi_{i j}\left(\omega_{l}\right)=\frac{1}{Z} \sum_{n, m} \frac{e^{-\beta E_{n}}-e^{-\beta E_{m}}}{\omega_{l}-\left(E_{n}-E_{m}\right)}\left\langle m\left|\boldsymbol{A}_{i}\right| n\right\rangle\left\langle n\left|\boldsymbol{A}_{j}\right| m\right\rangle,
$$

with $\omega_{\mathrm{k}}=2 \mathrm{l} \pi / \beta$.

Taking the peculiarities of the zeroth component explicitly into account we end up with:

$$
\begin{aligned}
\Pi_{i j}\left(\omega_{l}\right)= & \frac{1}{Z} \sum_{E_{n} \neq E_{m}} \frac{e^{-\beta E_{n}}-e^{-\beta E_{m}}}{\omega_{l}-\left(E_{n}-E_{m}\right)}\left\langle m\left|\boldsymbol{A}_{i}\right| n\right\rangle\left\langle n\left|\boldsymbol{A}_{j}\right| m\right\rangle \\
& +\delta_{l, 0} \frac{\beta}{Z} \sum_{E_{m}=E_{n}} e^{-\beta E_{n}}\left\langle m\left|\boldsymbol{A}_{i}\right| n\right\rangle\left\langle n\left|\boldsymbol{A}_{j}\right| m\right\rangle .
\end{aligned}
$$

For the self-adjoint operator it holds that $\left\langle m\left|\boldsymbol{A}_{i}\right| n\right\rangle=\left\langle n\left|\boldsymbol{A}_{i}\right| m\right\rangle$. The correlation function can be expressed in the so-called $\mathrm{Q}$-matrix formalism as:

$$
\boldsymbol{\Pi}\left(\omega_{\mathrm{k}}\right)=\mathbf{Q}^{+} \boldsymbol{\Lambda}\left(\omega_{\mathrm{k}}\right) \mathbf{Q},
$$


where the matrix $\mathbf{Q}$ is defined with the ordered index $\alpha=(n, m)$ as:

$$
\mathbf{Q}_{\alpha i}=\left\langle\mathrm{n}\left|\boldsymbol{A}_{\mathrm{i}}\right| \mathrm{m}\right\rangle
$$

and the diagonal matrix $\Lambda$ as:

$$
\Lambda_{\alpha \beta}\left(\omega_{l}\right)=\frac{\delta_{\alpha \beta}}{Z} \begin{cases}\frac{e^{-\beta E_{n}-e^{-\beta E_{m}}}}{\omega_{l}-\left(E_{n}-E_{m}\right)} & , E_{n} \neq E_{m}, \\ \delta_{l, 0} \beta e^{-\beta E_{n}} & , E_{n}=E_{m} .\end{cases}
$$

If all entries of one row in $Q$ are summed up one finds $\left\langle n\left|\sum_{i} A_{i}\right| m\right\rangle$. It was an assumption that the total quantity $\sum_{i} \boldsymbol{A}_{i}$ is conserved by the Hamiltonian and so has the same set of eigenstates. Thus, the matrix element is zero for $n \neq m$ and some eigenvalue $\lambda_{n}$ for $n=m$.

We will show in the following that the matrix $\Pi_{i j}\left(\omega_{l}\right)$ is singular for $l \neq 0$. This means that at least one of its eigenvalues is zero and so the determinant vanishes.

$\Pi_{i j}$ is a square matrix of size $N$ and can be seen as the product of two rectangular matrices $\left(\mathbf{Q}^{+} \boldsymbol{\Lambda}\right)$ and $\mathbf{Q}$. The former is of size $\mathbf{N} \times \mathrm{K}^{2}$ and the latter $\mathrm{K}^{2} \times \mathrm{N}$. It is obvious that $\mathrm{K}^{2}>\mathrm{N}$ for any reasonable system. The determinant of the product of such rectangular matrices can be written as the sum of the products of all corresponding major determinants of the factors [Haz06].

A major of a rectangular $\mathrm{N} \times \mathrm{K}^{2}$-matrix is the square matrix one gets by omitting $\mathrm{K}^{2}-\mathrm{N}$ columns (or rows in the case of a $\mathrm{K}^{2} \times \mathrm{N}$ ). It is a so-called maximum order square partitioned block. Furthermore, corresponding majors of a product like $\left(\mathbf{Q}^{+} \boldsymbol{\Lambda}\right) \cdot \mathbf{Q}$ are obtained by omitting columns respectively rows that have the same index. The proofs and a thorough discussion can be found in [Haz06].

So the determinant of $\Pi_{i j}\left(\omega_{l}\right)$ decomposes into a sum of corresponding major determinants. Consider a term where all rows of the major of $\mathbf{Q}$ belong to indices $\alpha=(n, m)$ from (B.4) with $n \neq m$. In this case the contribution to the determinant vanishes since according to the argument above all rows $\left\langle n\left|\sum_{i} \boldsymbol{A}_{i}\right| \mathrm{m}\right\rangle$ add up to zero. As a consequence the columns are linearly dependent which leads to a vanishing determinant. Thus, when $n \neq m$, all major determinants of $\mathbf{Q}$ are zero.

This is obviously not the case if rows with $n=m$ are part of the major. But then a factor $\delta_{l, 0}$ appears in the corresponding major determinant of $\left(\mathbf{Q}^{+} \boldsymbol{\Lambda}\right)$, which can be deduced from (B.5).

So one concludes that each term of the determinant $\left|\Pi\left(\omega_{l}\right)\right|$ vanishes except for the zeroth Matsubara frequency. With the introduction of some system-dependent constant $c$ it can be written as:

$$
\left|\Pi_{i j}\left(\omega_{l}\right)\right|=\delta_{l, 0} c
$$

Consequently, the correlation function matrix of self-adjoint operators is singular for $l \neq 0$ if the Hamiltonian commutates with the operator $\sum_{i} A_{i}$. 
This is easily seen for the special case of a homogeneous lattice with periodic boundary conditions, where the matrix is diagonalizable via a Fourier transformation to $k$-space. The $k=0$ component is then proportional to $\delta_{l, 0}$.

\section{B.2 IMPLiCATIONS OF THE SINGULAR MATRIX}

The fact that the correlation function $\Pi\left(\omega_{l}\right)$ can be singular under certain circumstances possibly poses a problem for the spin VCA. This can be seen when one recalls the derivation in sections 3.3 and 3.4. A similar situation arises in the EVCA for non-local particle density interactions [Ton05].

Suppose we have a lattice or cluster of $\mathrm{N}$ spins with a total $\mathbf{S}^{z}$ conserving Heisenberg Hamiltonian. According to the reasoning above, the longitudinal correlation function matrix $\Pi^{z}\left(\omega_{l}\right)$ is singular for $l \neq 0$. This also holds for all components of $\Pi\left(\omega_{l}\right)$ in case we have SU(2)symmetry.

In the equations derived for the SVCA, terms like $\operatorname{Tr} \ln \Pi^{z}\left(\omega_{l}\right)$ appear, for example in the free energy (3.34). Here, the trace goes over space indices and Matsubara frequencies. Unless $l=0$, the matrix $\Pi^{z}\left(\omega_{l}\right)$ is singular and so the logarithm respectively the term in question is undefined.

To examine if this really constitutes a problem we have to look at the relevant part of the free energy (3.34) where the terms $\ln \Pi^{z}$ and $\left(\Pi^{z}\right)^{-1}$ appear:

$$
-\operatorname{Tr} \ln \left(\left(\boldsymbol{\Pi}_{\mathrm{c}}^{z}\right)^{-1}-\mathbf{V}^{z}\right)^{-1}+\operatorname{Tr} \ln \Pi_{\mathrm{c}}^{z}=\operatorname{Tr} \ln \left(1-\mathbf{V}^{z} \boldsymbol{\Pi}_{\mathrm{c}}^{z}\right) .
$$

Here, we rewrote the expression by combining the logarithms on the left hand side. It is easy to see that the matrix inside the logarithm on the right is not singular and so the term itself is well defined. This means that the two traces on the left taken together must be well defined, too. This can be seen in the following way.

One can add a small regularization $\epsilon \equiv \epsilon \cdot \mathbb{1}$ to the correlation function in the second term on the left of (B.7):

$$
\sum_{\omega_{l}} \operatorname{Tr} \ln \left(\Pi^{z}\left(\omega_{l}\right)+\epsilon\right)
$$

Here, we wrote the summation over the Matsubara frequencies explicitly and the trace is now over space indices only. We can diagonalize 
the operator inside the trace to get the logarithm of the eigenvalues of $\Pi^{z}\left(\omega_{l}\right)$ which we denote as $\lambda_{m}\left(\omega_{l}\right)$ [Haz06]:

$$
\begin{aligned}
\sum_{\omega_{l}} \operatorname{Tr} \ln \left(\lambda_{\mathrm{m}}\left(\Pi^{z}\left(\omega_{l}\right)+\epsilon\right)=\right. & \sum_{\omega_{l}} \sum_{m} \ln \left(\lambda_{\mathrm{m}}\left(\omega_{l}\right)+\epsilon\right) \\
= & \sum_{\omega_{l}} \sum_{\lambda_{\mathrm{m}} \neq 0} \ln \left(\lambda_{\mathrm{m}}\left(\omega_{l}\right)+\epsilon\right) \\
& +\sum_{\omega_{l}} \ln \left(\delta_{l, 0} \lambda+\epsilon\right) .
\end{aligned}
$$

A singular matrix has one zero eigenvalue if it is non-degenerate which is the case for the correlation matrix in reasonable systems. In the last line of (B.9) we separated the terms which belong to this $\lambda_{m}\left(\omega_{l}\right)$. Given the discussion that lead to (B.6), we can rewrite it in the form $\left(\delta_{l, 0} \cdot \lambda\right)$. Here, $\lambda$ is the eigenvalue for the zeroth Matsubara mode. So except for this $l=0$ term there appear the logarithms $\ln (\epsilon)$ in (B.9). These diverge in the limit $\epsilon \rightarrow 0$ while all other terms are well-defined.

At first sight these singularities seem to be only a reformulation of the already known problem. Yet, they are lifted by the first term in (B.7). As was discussed in section 3.4, the matrix $\left(\left(\Pi_{\mathcal{c}}^{z}\right)^{-1}-V^{z}\right)^{-1}$ inside the logarithm can also be written in a form $\mathbf{Q}^{+} \boldsymbol{\Lambda}_{\mathrm{A}} \mathrm{Q}$ like (B.3). The $\mathrm{Q}$-matrices are the ones from $\Pi_{\mathcal{c}}^{z}$ while $\Lambda_{A}$ is different. Given this form the same arguments hold. The matrix is singular and can even be written analogously to (B.9). Naturally, the eigenvalues are different but the last sum can still be written as $\sum_{\omega_{l}} \ln \left(\delta_{l, 0} \lambda_{A}+\epsilon\right)$.

This means that the first trace of (B.7) has the same number of diverging terms $\ln (\epsilon)$, yet with a negative sign. Thus, all singularities cancel out and the free energy (3.34) is well-defined.

So, while the SVCA equations as a whole are sound one has to take care when working with single terms like $\operatorname{Tr} \ln \Pi^{z}\left(\omega_{l}\right)$. In such cases, the small regularization parameter needs to be added formally. In the limit $\epsilon \rightarrow 0$, though, it will have no effect when the free energy is evaluated and all diverging terms cancel out. But one has to keep the need for a regularization in mind when the inverse or the logarithm of singular matrices $\Pi^{z}$ appear in the derivation in chapter 3, not only for the free energy (3.34). 


\section{THE INTERACTION MATRIX IN FOURIER REPRESENTATION}

For the spin VCA we need to work with solvable reference systems. Throughout this thesis these are certain cluster tilings of the original lattice. Each of the individual subsystems can be dealt with using a suitable solver. However, a reference system is given by the complete superlattice of equivalent clusters. In this appendix we will give a short introduction of how to conveniently treat such a lattice with regard to the interaction quantities from chapters 3 and 4 . A more general discussion on cluster decompositions of lattices can be found in the lecture paper by Sénéchal [Sen08].

An example for a reference system is given in fig. C.1. Here, a onedimensional spin chain is tiled into two-site clusters. The spins of the original lattice interact via an exchange $J_{i j}$, while on a cluster the interaction is $\left(J_{c}\right)_{i j}$. Suppose the system a) consists of $N$ sites with periodic boundary conditions. Hence, it is translationally invariant and the interaction matrix $\mathrm{J}$ is diagonal in momentum space. It can be conveniently represented by $J(k)$ using the Fourier transformation:

$$
\mathrm{J}_{i j}=\frac{1}{\mathrm{~N}} \sum_{\mathbf{k}} \mathrm{e}^{\mathrm{ik} \cdot\left(\mathbf{r}_{\mathrm{i}}-\mathbf{r}_{\mathrm{j}}\right)} \mathrm{J}(\mathbf{k}) .
$$

Here, $k$ is the wave vector in momentum space and $\boldsymbol{r}_{i}$ denotes the position of site $i$ in real space. The tiling $b$ ) from fig. C.1 is only invariant with regards to the superlattice of identical cluster, i.e under a translation of two sites.

In the general case, the original lattice is tiled into clusters of $N_{c}$ sites each. One can introduce $N / N_{c}$ real space vectors $r_{m}$ of the superlattice, while the sites of a cluster are labelled by $\boldsymbol{r}_{\mathrm{a}}$. So the original lattice is uniquely represented as $r_{i}=r_{m}+r_{a}$ and each index $i$ can be replaced by the compound $(m, a)$. It is useful to introduce a discrete partial Fourier transformation of the superlattice [Sen08]. It closes under the identity $\sum_{\tilde{k}} e^{i \tilde{k} \cdot r_{m}}=\delta_{m, 0}$ whereas the wave vector $\tilde{k}$ belongs to a reduced Brillouin zone of size $N / N_{c}$. One ends up with a mixed representation characterized by $\tilde{k}$ and the site indices $a$.

Under such a transformation all cluster system quantities are independent of the reduced wave vector while the exchange $J_{i j}$ of the original lattice is not:

$$
J_{a b}(\tilde{\mathbf{k}})=\sum_{m} e^{i \tilde{\mathbf{k}} \cdot \boldsymbol{r}_{m}} J_{i j}
$$




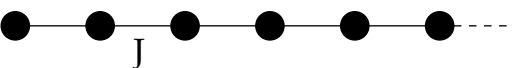

a)

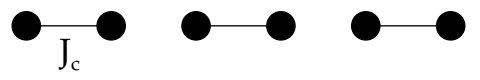

b)

Figure C.1: Part a) depicts a segment of a spin chain. A suitable reference model with two site clusters is shown in figure b). This can be seen as a meta-lattice consisting of the individual clusters.

with $i=(0, a)$ and $j=(m, b)$. For the example depicted in fig. C.1 with $\mathrm{N}_{\mathrm{c}}=2$ and first-neighbour interaction only, we find the matrix $\mathrm{J}$ from (C.2) with regards to the site indices $a$ and $b$ :

$$
\mathbf{J}(\tilde{\mathbf{k}})=\mathbf{J}\left(\begin{array}{cc}
0 & 1+e^{2 i \tilde{\mathbf{k}}} \\
1+e^{-2 i \tilde{k}} & 0
\end{array}\right) .
$$

This representation is convenient for the spin variational cluster approximation and the evaluation of the free energy (3.34). The spatial traces transform into sums over the reduced wave vector and the cluster sites indices. The former can be dealt with in several ways, i.e using numerical integration or discrete summation over a grid of $\mathrm{N} / \mathrm{N}_{\mathrm{c}}$ points covering the reduced Brillouin zone [Sen08]. Any further computation can be done entirely on the cluster.

We conclude this appendix by listing the mixed representations (C.2) of interaction matrices $\mathbf{V}^{\xi}=\mathbf{J}^{\xi}-\mathbf{J}_{\mathbf{c}}^{\xi}$ used in this thesis. The $\xi$ denotes $t$ and $z$ for transversal and longitudinal interactions. As in the main text we use $\mathrm{k}$ instead of $\tilde{\mathrm{k}}$ for the reduced wave vector.

In section 4.1 and 4.2 we applied two-, four- and six-site cluster reference systems with nearest-neighbour exchange to evaluate the SVCA free energy (4.2). For the computations the following interaction matrices have been used:

$$
\begin{aligned}
& \mathbf{V}_{2}^{\xi}(\mathbf{k})=\left(\begin{array}{cc}
0 & J^{\xi}\left(1+e^{2 i k}\right)-J_{\mathcal{C}}^{\xi} \\
J^{\xi}\left(1+e^{-2 i k}\right)-J_{\mathcal{C}}^{\xi} & 0
\end{array}\right), \\
& \mathbf{V}_{4}^{\xi}(\mathbf{k})=\left(\begin{array}{cccc}
0 & \left(J^{\xi}-J_{c}^{\xi}\right) & 0 & J^{\xi} e^{4 i k} \\
\left(J^{\xi}-J_{c}^{\xi}\right) & 0 & \left(J^{\xi}-J_{c}^{\xi}\right) & 0 \\
0 & \left(J^{\xi}-J_{c}^{\xi}\right) & 0 & \left(J^{\xi}-J_{c}^{\xi}\right) \\
J^{\xi} e^{-4 i k} & 0 & \left(J^{\xi}-J_{c}^{\xi}\right) & 0
\end{array}\right) \text {, } \\
& \mathbf{V}_{6}^{\xi}(\mathbf{k})= \\
& \left(\begin{array}{cccccc}
0 & \left(J^{\xi}-J_{\mathfrak{c}}^{\xi}\right) & 0 & 0 & 0 & J^{\xi} e^{6 i k} \\
\left(J^{\xi}-J_{\mathcal{c}}^{\xi}\right) & 0 & \left(J^{\xi}-J_{c}^{\xi}\right) & 0 & 0 & 0 \\
0 & \left(J^{\xi}-J_{c}^{\xi}\right) & 0 & \left(J^{\xi}-J_{c}^{\xi}\right) & 0 & 0 \\
0 & 0 & \left(J^{\xi}-J_{\mathfrak{c}}^{\xi}\right) & 0 & \left(J^{\xi}-J_{\mathfrak{c}}^{\xi}\right) & 0 \\
0 & 0 & 0 & \left(J^{\xi}-J_{\mathcal{c}}^{\xi}\right) & 0 & \left(J^{\xi}-J_{c}^{\xi}\right) \\
J^{\xi} e^{-6 i k} & 0 & 0 & 0 & \left(J^{\xi}-J_{\mathfrak{c}}^{\xi}\right) & 0
\end{array}\right) .
\end{aligned}
$$


In section 4.3 we presented results for the frustrated spin chain with competing interactions $\mathrm{J}_{1}$ between first- and $\mathrm{J}_{2}$ between second-neighbour sites. These were obtained by a reference system with six-spin clusters and the following interaction matrix:

$$
\begin{aligned}
& \mathbf{V}_{\mathrm{F}}^{\xi}(\mathbf{k})= \\
& \left(\begin{array}{cccccc}
0 & \left(J_{1}^{\xi}-J_{c, 1}^{\xi}\right) & \left(J_{2}^{\xi}-J_{c, 2}^{\xi}\right) & 0 & J_{2}^{\xi} e^{6 i k} & J_{1}^{\xi} e^{6 i k} \\
\left(J_{1}^{\xi}-J_{c, 1}^{\xi}\right) & 0 & \left(J_{1}^{\xi}-J_{c, 1}^{\xi}\right) & \left(J_{2}^{\xi}-J_{c, 2}^{\xi}\right) & 0 & J_{2}^{\xi} e^{6 i k} \\
\left(J_{2}^{\xi}-J_{c, 2}^{\xi}\right) & \left(J_{1}^{\xi}-J_{c, 1}^{\xi}\right) & 0 & \left(J_{1}^{\xi}-J_{c, 1}^{\xi}\right) & \left(J_{2}^{\xi}-J_{c, 2}^{\xi}\right) & 0 \\
0 & \left(J_{2}^{\xi}-J_{c, 2}^{\xi}\right) & \left(J_{1}^{\xi}-J_{c, 1}^{\xi}\right) & 0 & \left(J_{1}^{\xi}-J_{c, 1}^{\xi}\right) & \left(J_{2}^{\xi}-J_{c, 2}^{\xi}\right) \\
J_{2}^{\xi} e^{-6 i k} & 0 & \left(J_{2}^{\xi}-J_{c, 2}^{\xi}\right) & \left(J_{1}^{\xi}-J_{c, 1}^{\xi}\right) & 0 & \left(J_{1}^{\xi}-J_{c, 1}^{\xi}\right) \\
J_{1}^{\xi} e^{-6 i k} & J_{2}^{\xi} e^{-6 i k} & 0 & \left(J_{2}^{\xi}-J_{c, 2}^{\xi}\right) & \left(J_{1}^{\xi}-J_{c, 1}^{\xi}\right) & 0
\end{array}\right) .
\end{aligned}
$$

One can in principle also introduce easy-axis anisotropy terms a $\left(\mathbf{S}^{z}\right)^{2}$ within the SVCA. In such a case entries \pm a would appear along the diagonal of the longitudinal interaction matrix, where the sign depends on whether they are part of $\mathrm{J}^{\xi}$ or $\mathrm{J}_{\mathrm{c}}^{\xi}$. 



\section{SVCA EXTENSION TO VARIATIONAL MAGNETIC FIELDS}

The SVCA was introduced in chapter 3 in such a way that local magnetic fields could not be used as variational parameters. This is due to the fact that they appear as part of the non-interacting Hamiltonian. Given the derivation of the spin Luttinger-Ward functional in sections 3.1 and 3.2, this needs to be kept constant.

We thoroughly discussed in section 3.5 and chapter 5 that missing local fields as variational parameters might contribute to certain problems associated with the formalism at the moment. Apart from that, the ability to introduce magnetic fields in the variation would in general enhance the SVCA.

In this appendix, we present a possible solution to extend the approach in such a way. Yet, the arguments of the derivation are less robust than the original formalism and the resulting variational equations have not been sufficiently tested. In this sense, the scheme in the appendix should be viewed as preliminary.

We start with the functional (3.8) of the action of a Heisenberg system with a magnetic field:

$$
\begin{aligned}
& \tilde{S}\left[s^{\eta}, \tilde{\mathbf{J}}^{z z}, \tilde{\mathbf{J}}^{-+}, \tilde{\mathbf{h}}\right]=\mathrm{B}\left(s_{i}^{\eta}\right)+\int_{0}^{\beta} \mathrm{d} \tau \sum_{i}(\tilde{\mathbf{h}})_{i} s_{i}^{z}(\tau) \\
& +\int_{0}^{\beta} \mathrm{d} \tau \int_{0}^{\beta} \mathrm{d} \tau^{\prime} \sum_{i j} s_{i}^{z}(\tau)\left(\tilde{\mathbf{J}}^{z z}\right)_{i j}\left(\tau-\tau^{\prime}\right) s_{j}^{z}\left(\tau^{\prime}\right) \\
& +\int_{0}^{\beta} \mathrm{d} \tau \int_{0}^{\beta} \mathrm{d} \tau^{\prime} \sum_{i j}\left(s_{i}^{x}(\tau)\left(\tilde{\mathbf{J}}^{-+}\right)_{i j}\left(\tau-\tau^{\prime}\right) s_{j}^{x}\left(\tau^{\prime}\right)\right. \\
& \left.+s_{i}^{y}(\tau)\left(\tilde{\mathbf{J}}^{-+}\right)_{i j}\left(\tau-\tau^{\prime}\right) s_{j}^{y}\left(\tau^{\prime}\right)\right) .
\end{aligned}
$$

Here, we included an auxiliary field $\tilde{\mathrm{h}}$ which becomes the magnetic field $\mathbf{h}$ for the physical system. It is introduced as a diagonal matrix $\tilde{\mathbf{h}}=\tilde{h}_{i} \delta_{i i}$ in the site indices. Given the path integral representation (3.5) for the partition function, we find in analogy to (3.6):

$$
\begin{aligned}
\tilde{\mathbf{M}}_{\boldsymbol{i}}(\tau) & =\left\langle\mathbf{S}_{\mathfrak{i}}^{z}(\tau)\right\rangle_{\tilde{S}}=-\beta \frac{\delta \tilde{F}[\tilde{\mathbf{J}}, \tilde{\mathbf{h}}]}{\delta \tilde{\mathbf{h}}_{i}} \\
& =\frac{1}{\tilde{Z}} \int \prod_{i} \mathcal{D} \vec{s}_{\mathfrak{i}}\left(s_{i}^{z}(\tau) e^{-\tilde{S}}\right) .
\end{aligned}
$$

Hence, we have introduced the magnetization functional as the derivative of the free energy with respect to the auxiliary field. Using this rela- 
tion (D.2), we can extend the Legendre transform (3.14)-(3.16) of the free energy to include the diagonal matrix $\tilde{M}$ :

$$
\begin{aligned}
\tilde{\mathrm{A}}[\tilde{\boldsymbol{\Pi}}, \tilde{\mathbf{M}}] & =\tilde{\mathrm{F}}[\tilde{\mathbf{J}}, \tilde{\mathbf{h}}]+\frac{1}{\beta} \operatorname{Tr}(\tilde{\boldsymbol{\Pi}} \tilde{\mathbf{J}})-\operatorname{Tr}\left(\frac{\delta \tilde{\mathrm{F}}[\tilde{\mathbf{J}}, \tilde{\mathbf{h}}]}{\delta \tilde{\mathbf{h}}_{\mathbf{i}}} \tilde{\mathbf{h}}\right) \\
& =\tilde{\mathrm{F}}[\tilde{\mathbf{J}}, \tilde{\mathbf{h}}]+\frac{1}{\beta} \operatorname{Tr}(\tilde{\boldsymbol{\Pi}} \tilde{\mathbf{J}})+\frac{1}{\beta} \operatorname{Tr}(\tilde{\mathbf{M}} \tilde{\mathbf{h}}) .
\end{aligned}
$$

From this point on, the approach presented in this appendix follows the scheme from chapter 3 with additional functional terms associated with the magnetization. The introduction of the auxiliary field $\tilde{h}$ in the action and the subsequent functional derivations means that we are only left with the Berry phase $B\left(s_{i}^{\eta}\right)$ as the 'non-interacting' part in (D.1). In fact, we effectively give up this concept by making the Legendre transform (D.3) and subsequently the Luttinger-Ward functional dependent on all parameters of the Hamiltonian. This is similar to some applications of the EVCA by Tong [Ton05].

Such a procedure is not necessarily problematic since we do not develop the approach perturbatively. Yet, if we omit the Zeeman term $h \sum_{i} S_{i}^{z}$ as the non-interacting Hamiltonian, we change the prerequisites of the spin diagram technique (see section 2.2). This questions the physical meaning of the spin self-energies (3.20), who are defined in a perturbative way within the formalism. It is not clear at the moment how serious the consequences are since we do not change the diagrammatic expansion itself by varying the magnetic field. So, the spin self-energies can still be defined in the same way, but with a dependency on $\tilde{h}$. We do not take this subtlety into account explicitly in the derivation of the extended SVCA equations.

A more serious issue is the introduction of a 'self-energy' of the magnetization functional. Such a quantity is needed to establish a suitable Luttinger-Ward functional. In comparison to the spin correlation functions and their respective Larkin self-energies, the following is only a formal definition:

$$
\mathbf{X}=\mathbf{h}+\mathbf{M}^{-1}
$$

Similarities to this quantity can be seen in the generating functional approach by Izyumov et al. [IC01], yet one can not attribute a physical or perturbative meaning to it. Nevertheless, we continue extending the SVCA along this path.

Keeping the functional derivative $\beta \delta \tilde{A} / \delta \tilde{M}=\tilde{\mathbf{h}}$ in mind and using (D.4), we can define the extended spin Luttinger-Ward functional:

$$
\tilde{\Phi}[\tilde{\Pi}, \tilde{\mathbf{M}}]=\beta \tilde{\AA}[\tilde{\Pi}, \tilde{\mathbf{M}}]+\operatorname{Tr} \ln \tilde{\Pi}+\operatorname{Tr} \ln \tilde{\boldsymbol{M}} .
$$

For (D.5), $\delta \tilde{\Phi} / \delta \tilde{M}=\tilde{X}$ holds as well as the derivatives (3.25) with respect to the correlation functions. We continue by introducing a Legendre transform of the Luttinger-Ward functional (D.5):

$$
\tilde{\mathrm{P}}[\tilde{\boldsymbol{\Gamma}}, \tilde{\mathbf{X}}]=\tilde{\Phi}[\tilde{\boldsymbol{\Pi}}, \tilde{\mathbf{M}}]-\operatorname{Tr}(\tilde{\boldsymbol{\Gamma}} \tilde{\boldsymbol{\Pi}})-\operatorname{Tr}(\tilde{\mathbf{X}} \tilde{\mathbf{M}}),
$$


to express the free energy as a functional of the spin self-energies as well as $\tilde{X}$ :

$$
\beta \tilde{\mathrm{F}}_{\mathrm{SVCA}}[\tilde{\boldsymbol{\Gamma}}, \tilde{\mathbf{X}}]=\tilde{\mathrm{P}}[\tilde{\boldsymbol{\Gamma}}, \tilde{\mathbf{X}}]+\operatorname{Tr} \ln (\tilde{\boldsymbol{\Gamma}}-\mathbf{J})+\operatorname{Tr} \ln (\tilde{\mathbf{X}}-\mathbf{h}) .
$$

This is done in analogy to the derivation in section 3.3. The stationary conditions (3.31) and:

$$
\begin{gathered}
\left.\frac{\delta \tilde{\mathrm{F}}_{\mathrm{SVCA}}[\tilde{\boldsymbol{\Gamma}}, \tilde{\mathrm{X}}]}{\delta \tilde{\mathbf{X}}}\right|_{\tilde{\mathbf{X}}=\mathrm{X}}=0, \\
\tilde{\mathrm{F}}_{\mathrm{SVCA}}[\tilde{\Gamma}=\Gamma, \tilde{\mathbf{X}}=\tilde{\mathbf{X}}]=\mathrm{F}
\end{gathered}
$$

hold. We can now introduce a reference system to obtain the extended SVCA equations by eliminating the Luttinger-Ward functional. Finally, we end up with the approximate free energy as a function of the parameters of the cluster system:

$$
\beta F_{S V C A}\left(J_{c}, h_{c}\right)=\beta F_{c}+\operatorname{Tr} \ln \left(\frac{\Gamma_{c}-J}{\Gamma_{c}-J_{c}}\right)+\operatorname{Tr} \ln \left(\frac{\mathbf{X}_{c}-\mathbf{h}}{\mathbf{X}_{c}-\mathbf{h}_{c}}\right) .
$$

This is an extended version of (3.33). Due to the last term we are able to vary local fields in $\mathbf{h}_{\mathbf{c}}$. Indeed, we exactly reproduce (3.33) if $\mathbf{h}_{\mathbf{c}}$ is set to be $\mathbf{h}$ of the original system since the all quantities in the last term of (D.9) are diagonal. So, the scheme is consistent if we choose not to use magnetic fields as variational parameters.

The second term in (D.9) is a compact notation of the transversal and longitudinal part and can be evaluated in the way laid out in section 3.4. We can use the quantities $\mathrm{K}^{\mathrm{t}}$ from (3.43) and $\mathrm{K}^{z}$ from (3.47). The new term needs to be evaluated in a different way, which turns out to be less elaborate because all quantities involved are diagonal.

We start by inserting (D.4) for $\mathbf{X}_{\mathbf{c}}$ into the last term of (D.9):

$$
\begin{aligned}
\mathrm{K}^{\mathrm{h}}=\operatorname{Tr} \ln \left(\frac{\mathbf{X}_{\mathrm{c}}-\mathbf{h}}{\mathbf{X}_{\mathrm{c}}-\mathbf{h}_{\mathrm{c}}}\right) & =\operatorname{Tr} \ln \left(\mathbf{M}_{\mathbf{c}}\left[\left(\mathbf{M}_{\mathbf{c}}\right)^{-1}+\mathbf{h}_{\mathrm{c}}-\mathbf{h}\right]\right) \\
& =\operatorname{Tr} \ln \left(1-\Delta \mathbf{h} \mathbf{M}_{\mathbf{c}}\right),
\end{aligned}
$$

where we introduced $\Delta \mathbf{h}=\mathbf{h}-\mathbf{h}_{\mathbf{c}}$ in the second line. The trace in (D.10) goes over site indices and temperature $\tau$. The dependence of the magnetization $\left(\boldsymbol{M}_{\mathbf{c}}\right)_{i}=\left\langle\boldsymbol{S}_{i}^{z}(\tau)\right\rangle_{c}$ on $\tau$ can be easily integrated out. Additionally, since the matrices in (D.10) are diagonal we simply substitute the spatial trace by the sum over sites:

$$
\mathrm{K}^{\mathrm{h}}=\frac{\mathrm{N}}{\mathrm{N}_{\mathrm{c}}} \sum_{\mathrm{i}} \ln \left(1-\Delta \mathbf{h}_{\mathrm{i}} \frac{\beta}{Z} \sum_{n} e^{-\beta E_{n}}\left\langle\mathrm{n}\left|\boldsymbol{S}_{\mathrm{i}}^{z}\right| \mathrm{n}\right\rangle\right) .
$$

Here, $N$ and $N_{c}$ denote the number of sites of the original system respectively of a single cluster of the reference system. The $|n\rangle$ and $E_{n}$ are the eigenstates and -values of the cluster Hamiltonian.

Finally, to evaluate the extended free energy (D.9) one has to compute (3.43), (3.47) and (D.11):

$$
\beta F_{S V C A}\left(J_{c}, h_{c}\right)=\beta F_{c}+K^{t}+K^{z}+K^{h} .
$$


So, with (D.12) we present an extension of the SVCA to reference systems which include local fields as parameters. Yet, given the less sound nature of the magnetization 'self-energy' $X$ one needs to be careful with the applicability of the approximation. Up to now we only carried out inconclusive preliminary tests. On this basis we have not enough evidence to estimate the value of the extension and further investigations are needed. Hence, the scheme is included in the thesis as an appendix.

Nevertheless, the inclusion of local magnetic fields in the reference system should in principle be an improvement of the SVCA. Such parameters could help controlling the thermodynamical consistency of the magnetization, in the same way the chemical potential does for the particle number in fermionic and bosonic variational cluster approaches. Moreover, including local magnetic parameters in a reference system could be important for the treatment of phase transitions of spin systems. This especially includes regions where the SVCA breaks down and where the thermodynamical quantities like the magnetization show irregular and divergent behaviour (see section 4.2).

A further application for local variational terms would be models with a local order parameter. For example, one could introduce a staggered magnetic field to a reference system to treat Néel like phases in an antiferromagnetic spin model. The extension presented in this appendix in principle allows for such an approach. 


\section{BIBLIOGR A PHY}

[AGD63] A.A. Abrikosov, L.P. Gorkov, and I.E. Dzyaloshinski. Methods of quantum field theory in statistical mechanics. Dover, 1963 (cit. on p. 12).

[AL86] I. Affleck and E.H. Lieb. "A proof of part of Haldane's conjecture on spin chains." In: Condensed Matter Physics and Exactly Soluble Models. Springer, 1986, pp. 235-247 (cit. on p. 7).

[Aff ${ }^{+}$87] I. Affleck, T. Kennedy, E. H. Lieb, and H. Tasaki. "Rigorous results on valence-bond ground states in antiferromagnets." In: Physical Rev. Let. 59.7 (1987), p. 799 (cit. on p. 7).

[Aff $\left.{ }^{+} 88\right] \quad$ I. Affleck, T. Kennedy, E. H. Lieb, and H. Tasaki. "Valence bond ground states in isotropic quantum antiferromagnets." In: Commun. Math. Phys. 115 (1988), pp. 477-528 (cit. on p. 7).

[Aic ${ }^{+}$04] M. Aichhorn, H. G. Evertz, W. von der Linden, and M. Potthoff. "Charge ordering in extended Hubbard models: Variational cluster approach." In: Phys. Rev. B 70.23 (2004), p. 235107 (cit. on pp. 16, 54).

[Aic ${ }^{+}$06a] M. Aichhorn, E. Arrigoni, M. Potthoff, and W. Hanke. “Antiferromagnetic to superconducting phase transition in the hole- and electron-doped Hubbard model at zero temperature." In: Phys. Rev. B 74.2 (2006), p. 024508 (cit. on pp. 15, $48,62)$.

[Aic ${ }^{+}$06b] M. Aichhorn, E. Arrigoni, M. Potthoff, and W. Hanke. “Variational cluster approach to the Hubbard model: Phase- separation tendency and finite-size effects." In: Phys. Rev. B 74.23 (2006), p. 235117 (cit. on pp. 15, 48, 49).

[ADP15] F. Alet, K. Damle, and S. Pujari. “Sign-problem-free Monte Carlo simulation of certain frustrated quantum magnets." In: arXiv preprint arXiv:1511.01586 (2015) (cit. on pp. 8, 77).

[AA88a] D.P. Arovas and A. Auerbach. "Functional integral theories of low-dimensional quantum Heisenberg models." In: Phys. Rev. B 38.1 (1988), pp. 316-332 (cit. on p. 28).

[AKL11] E. Arrigoni, M. Knap, and W. von der Linden. "Extended self-energy functional approach for strongly correlated lattice bosons in the superfluid phase." In: Phys. Rev. B 84.1 (2011), p. 014535 (cit. on pp. 16, 55). 
[Arr ${ }^{+}$09] E. Arrigoni, M. Aichhorn, M. Daghofer, and W. Hanke. "Phase diagram and single-particle spectrum of $\mathrm{CuO}_{2}$ high$\mathrm{T}_{c}$ layers: variational cluster approach to the three-band Hubbard model." In: New Journal of Physics 11.5 (2009), p. 055066 (cit. on p. 16).

[AA88b] A. Auerbach and D.P. Arovas. "Spin Dynamics in the Square-Lattice Antiferromagnet." In: Phys. Rev. Lett. 61.5 (1988), pp. 617-620 (cit. on p. 28).

[Azz93] M. Azzouz. "Interchain-coupling effect on the one-dimensional spin-1/2 antiferromagnetic Heisenberg model." In: Phys. Rev. B 48.9 (1993), pp. 6136-6140 (cit. on p. 26).

[Bae ${ }^{+95] \quad D . ~ B a e r i s w y l, ~ D . K . ~ C a m p b e l l, ~ J . M . P . ~ C a r m e l o, ~ F . ~ G u i n e a, ~}$ and E. Louis. The Hubbard model: its physics and mathematical physics. Vol. 343. Springer Science \& Business Media, 1995 (cit. on p. 4).

[BHP08] M. Balzer, W. Hanke, and M. Potthoff. "Mott transition in one dimension: Benchmarking dynamical cluster approaches." In: Phys. Rev. B 77.4 (2008), p. 045133 (cit. on p. 16).

[BP10] M. Balzer and M. Potthoff. "Variational cluster approach to ferromagnetism in infinite dimensions and in one-dimensional chains." In: Phys. Rev. B 82.17 (2010), p. 174441 (cit. on p. 16).

[BO01] C. D. Batista and G. Ortiz. "Generalized Jordan-Wigner Transformations." In: Phys. Rev. Lett. 86 (2001), pp. 10821085 (cit. on pp. 26, 27).

[Bax82] R.J. Baxter. Exactly Solved Models in Statistical Mechanics. London: Academic Press, 1982 (cit. on pp. 26, 58).

[Bay62] G. Baym. "Self-Consistent Approximations in Many-Body Systems." In: Physical Review 127 (1962), pp. 1391 -1401 (cit. on pp. 13, 22, 23, 36).

[BK61] G. Baym and L.P. Kadanoff. "Conservation Laws and Correlation Functions." In: Physical Review 124 (1961), pp. 287 -299 (cit. on pp. 9, 13, 17, 45).

[Ber84] M. V. Berry. "Quantal Phase Factors Accompanying Adiabatic Changes." In: Proceedings of the Royal Society of London. A. Mathematical and Physical Sciences 392.1802 (1984), pp. $45-57$ (cit. on p. 84).

[Bet31] H. Bethe. "Zur Theorie der Metalle." In: Z. Phys. 71.3-4 (1931), pp. 205-226 (cit. on pp. 26, 58).

[BDD58] C. Bloch and C. De Dominicis. "Un développement du potentiel de Gibbs d'un système quantique composé d'un grand nombre de particules." In: Nuclear Physics 7 (1958), pp. 459-479 (cit. on pp. 17, 22). 
[BF64] J.C. Bonner and M.E. Fisher. "Linear magnetic chains with anisotropic coupling." In: Physical Review 135.3A (1964), A640 (cit. on p. 66).

[CR04] D.C. Cabra and G.L. Rossini. "Numerical Jordan-Wigner approach for two-dimensional spin systems." In: Phys. Rev. B 69.18 (2004), p. 184425 (cit. on p. 26).

[CTDL91] C. Cohen-Tannoudji, B. Diu, and F. Laloe. Quantum Mechanics. Bd. 2. Wiley, 1991 (cit. on p. 5).

[CR12] M. Combescure and D. Robert. Coherent states and applications in Mathematical Physics. Springer, 2012 (cit. on pp. 79, $80)$.

[Dah ${ }^{+}$04] C. Dahnken, M. Aichhorn, W. Hanke, E. Arrigoni, and M. Potthoff. "Variational cluster approach to spontaneous symmetry breaking: The itinerant antiferromagnet in two dimensions." In: Phys. Rev. B 70.24 (2004), p. 245110 (cit. on pp. 15, 54).

[Der ${ }^{+}$03] D. Oleg Derzhko, T. Verkholyak, R. Schmidt, and J. Richter. "Square-lattice $s=1 / 2 \mathrm{XY}$ model and the Jordan-Wigner fermions: the ground-state and thermodynamic properties." In: Physica A: Statistical Mechanics and its Applications 320 (2003), pp. $407-428$ (cit. on p. 26).

[Der01] O. Derzhko. “Jordan-Wigner fermionization for spin-1/2 systems in two dimensions: A brief review." In: Journal of Physical Studies 5 (2001), pp. 49-64 (cit. on p. 26).

[Dir26] P.A.M. Dirac. "On the theory of quantum mechanics." In: Proceedings of the Royal Society of London A: Mathematical, Physical and Engineering Sciences. Vol. 112. 762. The Royal Society. 1926, pp. 661-677 (cit. on p. 2).

[Dob03] S.V. Dobrov. "On the spin-fermion connection." In: Journal of Physics A: Mathematical and General 36.39 (2003), p. L503 (cit. on p. 27).

[EAT94] S. Eggert, I. Affleck, and M. Takahashi. "Susceptibility of the spin $1 / 2$ Heisenberg antiferromagnetic chain." In: Physical review letters 73.2 (1994), p. 332 (cit. on p. 6).

[Ess ${ }^{+}$05] F.H.L. Essler, H. Frahm, F. Göhmann, A. Klümper, and V.E. Korepin. The one-dimensional Hubbard model. Cambridge University Press, 2005 (cit. on p. 4).

[FT81] L.D. Faddeev and L.A. Takhtajan. "What is the spin of a spin wave?" In: Physics Letters A 85.6-7 (1981), pp. 375-377 (cit. on p. 7).

[Faz99] P. Fazekas. Lecture Notes on Correlation and Magnetism. Series in Modern Condensed Matter Physics - Vol. 5. Singapore: World Scientific, 1999 (cit. on pp. 2-7, 65). 
[FMH90] P. Fazekas and E. Müller-Hartmann. In: Z. Phys. B 78 (1990), p. 69 (cit. on pp. 28, 29).

[FW71] A. Fetter and J. Walecka. Quantum Theory of Many-Particle Systems. International Series in Pure and Applied Physics. New York: McGraw-Hill, 1971 (cit. on pp. 9, 41, 50).

[FP10] S. Filor and T. Pruschke. "A self-energy functional approach for spin systems." In: Journal of Physics: Conference Series 200.2 (2010), p. 022007 (cit. on pp. 10, 11, 31).

[FP14] S. Filor and T. Pruschke. "Variational cluster approximation to the thermodynamics of quantum spin systems." In: New Journal of Physics 16.6 (2014), p. 063059 (cit. on pp. 10, $35,57)$.

[Fra89] E. Fradkin. "Jordan-Wigner transformation for quantumspin systems in two dimensions and fractional statistics." In: Phys. Rev. Lett. 63 (3 1989), pp. 322-325 (cit. on p. 26).

[Fra91] E. Fradkin. Field Theories of Condensed Matter Systems. Addison-Wesley, 1991 (cit. on pp. 79, 82-84).

[FS88] E. Fradkin and M. Stone. "Topological terms in one- and two-dimensional quantum Heisenberg antiferromagnets." In: Phys. Rev. B 38 (1988), pp. 7215-7218 (cit. on pp. 37, 39, 79).

[GSF01] A. Georges, R. Siddharthan, and S. Florens. "Dynamical Mean-Field Theory of Resonating-Valence-Bond Antiferromagnets." In: Phys. Rev. Lett. 87.27 (2001), p. 277203 (cit. on pp. 16, 42).

[Geo ${ }^{+}$96] A. Georges, G. Kotliar, W. Krauth, and M. J. Rozenberg. "Dynamical mean-field theory of strongly correlated fermion systems and the limit of infinite dimensions." In: Rev. Mod. Phys. 68 (1996), p. 13 (cit. on p. 15).

[Gri05] D.J. Griffiths. Introduction to Quantum Mechanics. Pearson international edition. Pearson Prentice Hall, 2005 (cit. on p. 2).

[Gri64] R.B. Griffiths. "Magnetization Curve at Zero Temperature for the Antiferromagnetic Heisenberg Linear Chain." In: Phys. Rev. 133.3A (1964), A768-A775 (cit. on p. 63).

[Hal83] F.D.M. Haldane. "Nonlinear field theory of large-spin Heisenberg antiferromagnets: semiclassically quantized solitons of the one-dimensional easy-axis Néel state." In: Physical Review Letters 50.15 (1983), p. 1153 (cit. on p. 7).

[Haz06] A.K. Hazra. Matrix: Algebra, Calculus and Generalized Inverse, Part 1. Cambridge: Cambridge International Science Publishing, Limited, 2006 (cit. on pp. 88, 90). 
[HMHV06] F. Heidrich-Meisner, A. Honecker, and T. Vekua. "Frustrated ferromagnetic spin- $1 / 2$ chain in a magnetic field: the phase diagram and thermodynamic properties." In: Phys. Rev. B 74.2 (2006), p. 020403 (cit. on p. 70).

[Hei26] W. Heisenberg. "Many-body problem and resonance in Quantum Mechanics." In: Z. Phys. 38 (1926), pp. 411-426 (cit. on p. 2).

[Hon ${ }^{+}$16] A. Honecker, S. Wessel, R. Kerkdyk, T. Pruschke, F. Mila, and B. Normand. "Thermodynamic properties of highly frustrated quantum spin ladders: Influence of many-particle bound states." In: Phys. Rev. B 93.5 (2016), p. 054408 (cit. on pp. $8,69,77)$.

[Hub65] J. Hubbard. "Electron Correlations in Narrow Energy Bands. IV. The Atomic Representation." In: Proceedings of the Royal Society of London. Series A. Mathematical and Physical Sciences 285.1403 (1965), pp. 542-560 (cit. on pp. 4, 27).

[HZ93] L. Huerta and J. Zanelli. "Bose-Fermi transformation in three-dimensional space." In: Phys. Rev. Lett. 71 (1993), pp. 3622-3624 (cit. on p. 27).

[Hur82] C. M. Hurd. "Varieties of magnetic order in solids." In: Contemporary Physics 23.5 (1982), pp. 469-493 (cit. on p. 1).

[ID89] C. Itzykson and J.-M. Drouffe. Statistical Field Theory Vol.I $\mathcal{E}$ II. Cambridge: Cambridge University Press, 1989 (cit. on pp. 6, 9, 21).

[IC01] Y.A. Izyumov and N.I. Chashchin. "Spin dynamics of a Heisenberg ferromagnet in a wide temperature range: I. Generating functional method." In: Physics of metals and metallography 92.5 (2001), pp. 451-459 (cit. on pp. 24, 96).

[IKOS74] Y.A. Izyumov, F.A. Kassan-Ogly, and Y.N. Skryabin. Field methods in the theory of ferromagnetism. Science, Moscow, 1974 (cit. on pp. 17-20, 22-24).

[IS88] Y.A. Izyumov and Y.N. Skryabin. Statistical Mechanics of Magnetically Ordered Systems. New York: Plenum, 1988 (cit. on pp. 17-20, 22, 42, 74).

[Joh ${ }^{+}$00] D.C. Johnston, R.K. Kremer, M. Troyer, X. Wang, A. Klümper, S.L. Bud'ko, A.F. Panchula, and P.C. Canfield. "Thermodynamics of spin $S=1 / 2$ antiferromagnetic uniform and alternating-exchange Heisenberg chains." In: Physical Review B 61.14 (2000), p. 9558 (cit. on p. 6).

[JW28] P. Jordan and E. Wigner. "Über das Paulische Äquivalenzverbot." In: Z. Phys. A 47 (1928), pp. 631-651 (cit. on pp. 25, 62).

[Kar07] M. Kardar. Statistical physics of particles. Cambridge University Press, 2007 (cit. on p. 12). 
[KM84] H. Keiter and G. Morandi. "Thermodynamic perturbation theory for systems with strong local correlations." In: Physics Reports (Review Section of Physics Letters) 109 (1984), pp. 227-308 (cit. on p. 31).

[Kit87] C. Kittel. Quantum theory of solids. Second Revised Printing. Wiley, 1987 (cit. on p. 28).

[Klu93] A. Kluemper. "Thermodynamics of the anisotropic spin $1 / 2$ Heisenberg chain and related quantum chains." In: $Z$. Phys. B Condensed Matter 91.4 (1993), pp. 507-519 (cit. on p. 6).

[Klu98] A. Kluemper. "The spin-1/2 Heisenberg chain: thermodynamics, quantum criticality and spin-Peierls exponents." In: The European Physical Journal B 5 (1998), pp. 677-685 (cit. on pp. 6, 58, 61, 65, 66).

[KAL11] M. Knap, E. Arrigoni, and W. von der Linden. "Variational cluster approach for strongly correlated lattice bosons in the superfluid phase." In: Phys. Rev. B 83.13 (2011), p. 134507 (cit. on p. 55).

[KD06] W. Koller and N. Dupuis. "Variational cluster perturbation theory for Bose-Hubbard models." In: J. Phys.: Condens. Matter 18 (2006), pp. 9525-9540 (cit. on pp. 9, 16, 29, $30,33,48,50,52,54,55,73)$.

[KFG15] E. Kozik, M. Ferrero, and A. Georges. "Nonexistence of the Luttinger-Ward Functional and Misleading Convergence of Skeleton Diagrammatic Series for Hubbard-Like Models." In: Phys. Rev. Lett. 114.15 (2015), p. 156402 (cit. on p. 13).

[Kur83] Y. Kuramoto. "Self-Consistent Perturbation Theory for Dynamics of Valence Fluctuations I. Single-Site Theory." In: Z. Phys. B - Condensed Matter 53 (1983), pp. 37-52 (cit. on pp. 31, 32).

[LMM11] C. Lacroix, P. Mendels, and F. Mila. Introduction to Frustrated Magnetism: Materials, Experiments, Theory. Vol. 164. Springer Science \& Business Media, 2011 (cit. on pp. 6-8).

[Lan50] C. Lanczos. An iteration method for the solution of the eigenvalue problem of linear differential and integral operators.

United States Governm. Press Office Los Angeles, CA, 1950 (cit. on p. 77).

[Len+16] B. Lenz, S.R. Manmana, T. Pruschke, F.F. Assaad, and M. Raczkowski. "Mott Quantum Criticality in the Anisotropic 2D Hubbard Model." In: Physical review letters 116.8 (2016), p. 086403 (cit. on p. 16). 
[LW60] J. M. Luttinger and J. C. Ward. "Ground-State Energy of a Many-Fermion System. II." In: Physical Review 118.5 (1960), pp. 1417-1427 (cit. on pp. 13, 17, 23, 36, 43).

[Mai ${ }^{+}$05] T. A. Maier, M. Jarrell, T. Pruschke, and M. Hettler. "Quantum cluster theories." In: Rev. Mod. Phys. 77 (2005), p. 1027 (cit. on p. 9).

[MG69] C.K. Majumdar and D.K. Ghosh. “On Next-Nearest-Neighbor Interaction in Linear Chain. I." In: Journal of Mathematical Physics 10.8 (1969), pp. 1388-1398 (cit. on pp. 7, 8, 68).

[MM56] T. Matsubara and H. Matsuda. "A Lattice Model of Liquid Helium, I." In: Progress of Theoretical Physics 16.6 (1956), pp. 569-582 (cit. on p. 28).

[Mat88] D.C. Mattis. The theory of magnetism. Vol. 1. Harper \& Row New York, 1988 (cit. on pp. 25, 26, 29).

[Mat06] D.C. Mattis. The Theory of Magnetism Made Simple: An Introduction to Physical Concepts and to Some Useful Mathematical Methods. World Scientific, 2006 (cit. on pp. 1, 4, 5, 7).

[MW66] N. D. Mermin and H. Wagner. "Absence of Ferromagnetism or Antiferromagnetism in One- or Two-Dimensional Isotropic Heisenberg Models." In: Phys. Rev. Lett. 17.22 (1966), pp. 1133-1136 (cit. on p. 65).

[MK04] H.-J. Mikeska and A. K. Kolezhuk. “One-dimensional magnetism." In: Quantum magnetism. Springer, 2004, pp. 1-83 (cit. on pp. 7, 68).

[Mil73] W. Miller. Symmetry groups and their applications. Vol. 50. Academic Press, 1973 (cit. on p. 80).

[Nak98] T. Nakamura. "Vanishing of the negative-sign problem of quantum Monte Carlo simulations in one-dimensional frustrated spin systems." In: Phys. Rev. B 57.6 (1998), R3197R3200 (cit. on p. 8).

[NO88] J.W. Negele and H. Orland. Quantum Many-Particle Physics. Addison-Wesley, 1988 (cit. on pp. 17, 18, 36, 37, 82).

[NR09] W. Nolting and A. Ramakanth. Quantum theory of magnetism. Springer Science \& Business Media, 2009 (cit. on p. 5).

[OBS10] A.S. Ovchinnikov, I.G. Bostrem, and Vl.E. Sinitsyn. “Cluster perturbation theory for spin Hamiltonians." In: Theoretical and Mathematical Physics 162.2 (2010), pp. 179-187 (cit. on p. 53).

[OV04] S.G. Ovchinnikov and V.V. Valkov. Hubbard Operators in the Theory of Strongly Correlated Electrons. London: Imperial College Press, 2004 (cit. on pp. 9, 27).

[Per86] A. Perelomov. Generalized Coherent States and Their Applications. Springer-Verlag, 1986 (cit. on pp. 37, 39, 79). 
[PSS69] E. M. Pikalev, M.A. Savchenko, and J. Sólyom. "Thermodynamics and Correlation Functions of a Heisenberg Antiferromagnet." In: Soviet Phys. - JETP 28 (1969), p. 734 (cit. on pp. 17, 24, 42, 54, 74).

[PWE10] P. Pippan, S. R. White, and H. G. Evertz. "Efficient matrixproduct state method for periodic boundary conditions." In: Phys. Rev. B 81.8 (2010), p. 081103 (cit. on p. 77).

[Pot03a] M. Potthoff. "Self-energy-functional approach: Analytical results and the Mott-Hubbard transition." In: Eur. Phys. J. B 36 (2003), pp. 335-348 (cit. on pp. 12, 36, 48, 50, 73).

[Pot03b] M. Potthoff. "Self-energy-functional approach to systems of correlated electrons." In: Eur. Phys. J. B 32 (2003), p. 429 (cit. on pp. 9, 12, 13, 15, 16, 36, 73).

[Pot06a] M. Potthoff. "Non-perturbative construction of the Luttinger-Ward functional." In: Condens. Mat. Phys. 9 (2006), p. 557 (cit. on pp. 13, 36, 37, 42, 43).

[Pot06b] M. Potthoff. "Systematics of approximations constructed from dynamical variational principles." In: Effective Models for Low-Dimensional Strongly Correlated Systems. Vol. 816. 2006, pp. 41-54 (cit. on p. 46).

[Pot12a] M. Potthoff. "Self-Energy-Functional Theory." In: Strongly Correlated Systems: Theoretical Methods. Ed. by A. Avella and F. Mancini. Berlin, Heidelberg: Springer Berlin Heidelberg, 2012, pp. 303-339 (cit. on pp. 12-16, 23, 59, 74, 75).

[Pot12b] M. Potthoff. "Static and dynamic variational principles for strongly correlated electron systems." In: arXiv:1202.4907 (2012) (cit. on p. 12).

[PAD03] M. Potthoff, M. Aichhorn, and C. Dahnken. “Variational cluster approach to correlated electron systems in low dimensions." In: Phys. Rev. Lett. 91 (2003), p. 206402 (cit. on p. 15).

[PB07] M. Potthoff and M. Balzer. "Self-energy-functional theory for systems of interacting electrons with disorder." In: Phys. Rev. B 75.12 (2007), p. 125112 (cit. on p. 16).

[Rad71] J.M. Radcliffe. "Some properties of coherent spin states." In: J. Phys. A 4 (1971), pp. 313-323 (cit. on pp. 37, 38).

[RSH04] J. Richter, J. Schulenburg, and A. Honecker. "Quantum magnetism in two dimensions: From semi-classical Néel order to magnetic disorder." In: Quantum Magnetism. Vol. 645. Lecture Notes in Physics. Springer Berlin Heidelberg, 2004, pp. 85-153 (cit. on pp. 7, 68, 77).

[San10] A.W. Sandvik. "Computational Studies of Quantum Spin Systems." In: AIP Conference Proceedings 1297.1 (2010), pp. $135-338$ (cit. on pp. 6, 77). 
[Sch05] U. Schollwöck. "The density-matrix renormalization group." In: Rev. Mod. Phys. 77 (2005), p. 259 (cit. on p. 77).

[Sen08] D. Senéchal. "An introduction to quantum cluster methods." In: arXiv:0806.2690v2 (2008) (cit. on pp. 9, 34, 48, 50, $54,62,77,91,92)$.

[SS81] B. S. Shastry and B. Sutherland. "Exact ground state of a quantum mechanical antiferromagnet." In: Physica B+C 108.1 (1981), pp. 1069 -1070. ISSN: 0378-4363 (cit. on p. 69).

[SS96] Q. Si and J. L. Smith. "Kosterlitz-Thouless Transition and Short Range Spatial Correlations in an Extended Hubbard Model." In: Phys. Rev. Lett. 77 (1996), p. 3391 (cit. on pp. 16, 42).

[SS99] J. L. Smith and Q. Si. "Non-Fermi liquids in the two-band extended Hubbard model." In: EPL (Europhysics Letters) 45.2 (1999), p. 228 (cit. on p. 42).

[SS00] J. L. Smith and Q. Si. "Spatial correlations in dynamical mean field theory." In: Phys. Rev. B 61 (2000), p. 5184 (cit. on pp. 16, 42).

[SS06] J. Stöhr and H. C. Siegmann. Magnetism. Vol. 5. Solid-State Sciences. Springer, Berlin, Heidelberg, 2006 (cit. on p. 5).

[Sto86] M. Stone. "Born-Oppenheimer approximation and the origin of Wess-Zumino terms: Some quantum-mechanical examples." In: Phys. Rev. D 33 (1986), pp. 1191-1194 (cit. on p. 83).

[SK02] P. Sun and G. Kotliar. "Extended dynamical mean-field theory and GW method." In: Physical Review B 66.8 (2002), p. 085120 (cit. on p. 16).

[Tak09] M. Takahashi. Thermodynamics of One-Dimensional Solvable Models. Cambridge: Cambridge University Press, 2009 (cit. on pp. 6, 58).

[Tas98] H. Tasaki. "The Hubbard model - an introduction and selected rigorous results." In: Journal of Physics: Condensed Matter 10.20 (1998), p. 4353 (cit. on p. 4).

[Ton05] N.-H. Tong. "Extended Variational Cluster Approximation for Correlated Systems." In: Phys. Rev. B 72 (2005), p. 115104 (cit. on pp. 16, 26, 29, 30, 37, 42, 43, 73, 89, 96).

[TW05] M. Troyer and U.-J. Wiese. "Computational Complexity and Fundamental Limitations to Fermionic Quantum Monte Carlo Simulations." In: Phys. Rev. Lett. 94.17 (2005), p. 170201 (cit. on p. 8).

[UFM63] G.E. Uhlenbeck, G.W. Ford, and E.W. Montroll. Lectures in Statistical Physics. AMS, Providence, RI, 1963 (cit. on pp. 20, 23). 
[VLP68a] V.G. Vaks, A.I. Larkin, and S.A. Pikin. "Spin Waves and Correlation Functions in a Ferromagnetic." In: Soviet Phys. - JETP 26 (1968), p. 647 (cit. on pp. 21, 54).

[VLP68b] V.G. Vaks, A.I. Larkin, and S.A. Pikin. "Thermodynamics of an ideal ferromagnetic substance." In: Soviet Phys. - JETP 26 (1968), p. 188 (cit. on pp. 17, 18).

[VV32] J. H. Van Vleck. The theory of electric and magnetic susceptibilities. Oxford University Press, 1932 (cit. on pp. 2-4).

[Wic50] G. C. Wick. "The Evaluation of the Collision Matrix." In: Physical Review 80 (2 1950), pp. 268-272 (cit. on p. 17).

[Wie88] P. Wiegmann. "Superconductivity in Strongly Correlated Electronic Systems and Confinement versus Deconfinement Phenomenon." In: Phys. Rev. Lett. 60 (1988), p. 821 (cit. on p. 37).

[Wil65] J. H. Wilkinson. The algebraic eigenvalue problem. Vol. 87. Clarendon Press Oxford, 1965 (cit. on p. 77).

[Zac ${ }^{+}$02] M. G. Zacher, R. Eder, E. Arrigoni, and W. Hanke. “Evolution of the stripe phase as a function of doping from a theoretical analysis of angle-resolved photoemission data." In: Phys. Rev. B 65.4 (2002), p. 045109 (cit. on p. 48). 


\section{ACKNOWLEDGEMENTS}

First of all I would like to thank my late supervisor Prof. Dr. Thomas Pruschke, who sadly passed away much to soon. He gave me the opportunity to work on this thesis and remained supportive even when things got difficult. His door was always open and he was never bothered by any questions. He was an extraordinary supervisor and scientist, but above all a great human being.

I especially thank Prof. Dr. Stefan Kehrein and Dr. Salvatore Manmana, who took over as the thesis committee after Thomas' demise.

Dr. Kehrein also agreed to be the first reviewer of this thesis, for which I am very grateful. Prof. Dr. Andreas Honecker accepted the duty of being the second reviewer. For this and for the support through all those years I would like to thank our spin master.

I should not forget to mention Prof. Dr. Kurt Schönhammer, who was in my thesis committee when I started and whose comments where always helpful and appreciated.

Your are nothing without your loved ones. So I deeply thank my parents for all they did for me, my sister Susanne, my niece Anne and my nephews Peter and Hannes, my Annette.

And of course everybody else who is dear to me, my family and friends far and near.

This has been a long journey and I met many wonderful people along the way. Elham, Ehsan, Ebad, Fabi, Timo, Timo, Benni, Thomas, Florian, Navid - thanks to you guys for the coffee chit-chat, the serious and the absurd discussions, the funny and the sometimes not so funny jokes... Other people need to be mentioned, the list is in no kind of order nor complete: Martin, Jonny, Christoph, Andreas, Patrick, Ingo, Ansgar, Piet, Oliver, Rainer, Davide, Sebastian, Alexander, Nelli, Veronika, Ulrich, Johannes, Maria, René...

And I should also not forget to thank Katrin Glormann and Gabriele Schubert, the institute's secretaries, always help- and resourceful.

Finally, I thank everybody I forgot to thank! This is it!

Look me in the eye / And if you see familiarity Then celebrate the contradiction / Help me when I fall

To walk unafraid / I'll be clumsy instead Hold my love me or leave me high 
...Without a universal law there is no gravity Without a gravity there is no atmosphere Without an atmosphere there is no chance at life And with no chance at life...i don't exist...

- 13 \& God, Soft Atlas 